\title{
Pharmaceutical Oriented Selective Synthesis of Mononitriles and Dinitriles Directly from Methyl(hetero)arenes: Access to Chiral Nitriles and Citalopram
}

Jie Liu, ${ }^{\dagger}$ Hong-Xing Zheng, ${ }^{\dagger}$ Chuan-Zhi Yao, ${ }^{\dagger}$ Bing-Feng Sun, ${ }^{\ddagger}$, and Yan-Biao Kang ${ }^{\ddagger} * *$

†Center of Advanced Nanocatalysis, Department of Chemistry, University of Science and Technology of China, Hefei, Anhui 230026, China

‡ CAS Key Laboratory of Synthetic Chemistry of Natural Substances, Shanghai Institute of Organic Chemistry, CAS, 345 Lingling Road, Shanghai 200032, China

\section{E-mail: ybkang@ustc.edu.cn}

\section{Contents}

1. General information

2. Experimental procedures

$\mathrm{S} 1-\mathrm{S} 15$

3. References

S16

4. NMR spectra and HPLC Spectra

S17-S76

\section{General information}

Solvents were pre-dried over activated $4 \AA$ molecular sieves and heated to reflux over calcium hydride $\left(\mathrm{CH}_{3} \mathrm{CN}, \mathrm{DCM}, \mathrm{Et} 3 \mathrm{~N}, \mathrm{THF}\right)$ under argon atmosphere and collected by distillation. ${ }^{1} \mathrm{H},{ }^{13} \mathrm{C}$ NMR spectra were recorded on a Bruker 400 spectrometer; Chemical shifts are reported in $\delta$ units relative to $\mathrm{CDCl}_{3}\left[{ }^{1} \mathrm{H}\right.$ $\left.\delta=7.26,{ }^{13} \mathrm{C} \delta=77.00\right]$. HRMS were recorded by the mass spectrometry service at University of Science and Technology of China (Cl-35, Br-79). ICP-AES were examined by service at University of Science and Technology of China. Methylarenes and other chemicals without notes in experimental section were purchased from commercial sources.

\section{General procedures}

\subsection{General procedure for preparation of $t$-BuONO ${ }^{1}$}

To an ice-cold solution of (c) $\mathrm{H}_{2} \mathrm{SO}_{4}(22 \mathrm{~mL}, 0.4 \mathrm{~mol})$ in $\mathrm{H}_{2} \mathrm{O}(25 \mathrm{~mL})$ was added $t$ - $\mathrm{BuOH}(38 \mathrm{~mL}, 0.4$ mol) dropwise followed by the addition of a solution of sodium nitrite ( $42 \mathrm{~g}$, in $\left.100 \mathrm{~mL} \mathrm{of} \mathrm{H}_{2} \mathrm{O}\right)$. The reaction was kept at $0{ }^{\circ} \mathrm{C}$ during the addition, which was complete of $1 \mathrm{~h}$. The reaction mixture was warmed to r.t. and stirred overnight. The aquous layer was separated and the organic portion was washed with saturated aq. $\mathrm{NaHCO}_{3}$ solution and saturated aq. $\mathrm{NaCl}$ solution. The organic portion was then dried over $\mathrm{Na}_{2} \mathrm{SO}_{4}$ and purified by distillation. A light yellow fraction (ca. $20-30 \mathrm{~mL}$ ) was collected at $80-90{ }^{\circ} \mathrm{C}$ and used for all ammoxidation reactions. It was stored at $4{ }^{\circ} \mathrm{C}$. 


\subsection{Preparation of starting materials}

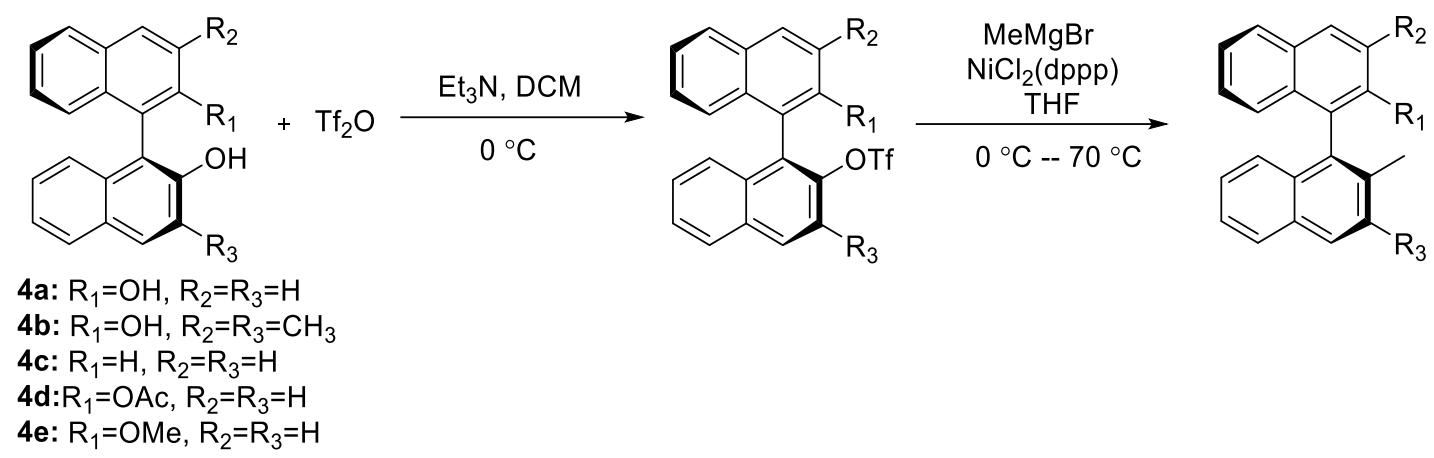

(S)-1,1'-bi-2-naphthol (2.0 g, $7 \mathrm{mmol}, 1.0$ equiv) was weighed into a $100 \mathrm{~mL}$ round-bottomed flask. After dried in vacuo for 15 min under argon atmosphere, and $20 \mathrm{~mL}$ dry DCM was added into the flaskto form a suspension. Then $\mathrm{Et}_{3} \mathrm{~N}$ ( $3 \mathrm{~mL}, 21 \mathrm{mmol}, 3.0$ equiv) was added into the mixture and the reaction mixture turned into a solution. Then $\mathrm{Tf}_{2} \mathrm{O}(3 \mathrm{~mL}, 17 \mathrm{mmol}, 2.4$ equiv) was added dropwise into the solution cooled by an ice-bath. During addition the solution turned into dark brown. After disppearing of the substrate on TLC, the reaction mixture was concentrated by rotary evaporation under reduced pressure and purified on silica gel column, eluented with petroleum ether and ethyl acetate to give the desired product.

The intermediate from the above step (1.0 equiv) was dried in vacuo for 15 min under argon atmosphere. Then $\mathrm{NiCl}_{2}$ (dppp) ( 0.05 equiv) was weighed into the flask in a glovebox, which was diluted by $30 \mathrm{~mL}$ of dry THF out of the glovebox. $\mathrm{MeMgBr}\left(6.0\right.$ equiv) (3 M) in $\mathrm{Et}_{2} \mathrm{O}$ was added dropwise into the solution cooled by an ice-bath. After addition, the mixture was warmed to room temperature, then was refluxed for $24 \mathrm{~h}$. After disppearing of the substrate on TLC, the reaction mixture was quenched by $1 \mathrm{M} \mathrm{HCl}$ aqueous solution. The aqueous layer was extracted with $\mathrm{Et}_{2} \mathrm{O}(30 \mathrm{~mL} \times 4)$, then the organic layer were combined, dried over anhydrous sodium sulfate, concentrated by rotary evaporation under reduced pressure and purified on silica gel column eluented with petroleum ether and ethyl acetate to give the desired products.<smiles>Cc1ccc2ccccc2c1-c1c(C)ccc2ccccc12</smiles>

(S)-2,2'-dimethyl-1,1'-binaphthalene (1e $)^{2}$

Prepared according to the general procedure, purified by flash column chromatography $(\mathrm{PE} / \mathrm{EA}=$ 100:1), white solid (2.0 g, 63\% yield). ${ }^{1} \mathbf{H} \mathbf{~ N M R ~}\left(\mathrm{CDCl}_{3}, 400 \mathrm{MHz}\right) \delta 7.90(\mathrm{t}, J=7.2 \mathrm{~Hz}, 4 \mathrm{H}), 7.52(\mathrm{~d}, J$ 
$=8.4 \mathrm{~Hz}, 2 \mathrm{H}), 7.40(\mathrm{t}, J=7.4 \mathrm{~Hz}, 2 \mathrm{H}), 7.21(\mathrm{t}, J=7.6 \mathrm{~Hz}, 2 \mathrm{H}), 7.05(\mathrm{~d}, J=8.4 \mathrm{~Hz} 2 \mathrm{H}), 2.05(\mathrm{~s}, 6 \mathrm{H})$.<smiles>Cc1cc2ccccc2c(-c2c(C)c(C)cc3ccccc23)c1C</smiles>

\section{(S)-2,2',3,3'-tetramethyl-1,1'-binaphthalene (1f $)^{2}$}

Prepared according to the general procedure, purified by flash column chromatography $(\mathrm{PE} / \mathrm{EA}=50: 1)$, light yellow solid (122.4 mg, 78\% yield). ${ }^{1} \mathbf{H} \mathbf{~ N M R}\left(\mathrm{CDCl}_{3}, 400 \mathrm{MHz}\right) \delta 7.81(\mathrm{~d}, J=8.0 \mathrm{~Hz}, 2 \mathrm{H}), 7.74$ $(\mathrm{s}, 2 \mathrm{H}), 7.35(\mathrm{t}, J=7.4 \mathrm{~Hz}, 2 \mathrm{H}), 7.12(\mathrm{t}, J=7.6 \mathrm{~Hz}, 2 \mathrm{H}), 6.95(\mathrm{~d}, J=8.4 \mathrm{~Hz} 2 \mathrm{H}), 2.54(\mathrm{~s}, 6 \mathrm{H}), 1.95(\mathrm{~s}$, $6 \mathrm{H})$.<smiles>COc1cccc(C)c1-c1c(C)cccc1OC</smiles>

(S)-2,2'-dimethoxy-6,6'-dimethyl-1,1'-biphenyl (1g) ${ }^{16,17}$

Prepared according to the literature procedure, purified by flash column chromatography $(\mathrm{PE} / \mathrm{EA}=$ 50:1), white solid (0.15 g, 88\% yield). ${ }^{1} \mathbf{H} \mathbf{N M R}\left(\mathrm{CDCl}_{3}, 400 \mathrm{MHz}\right) \delta 7.21(\mathrm{t}, J=7.6 \mathrm{~Hz}, 2 \mathrm{H}), 6.80(\mathrm{~d}, J$ $=7.2 \mathrm{~Hz}, 2 \mathrm{H}), 6.75(\mathrm{~d}, J=8.4 \mathrm{~Hz}, 2 \mathrm{H}), 3.82(\mathrm{~s}, 6 \mathrm{H}), 2.37(\mathrm{~s}, 6 \mathrm{H})$.

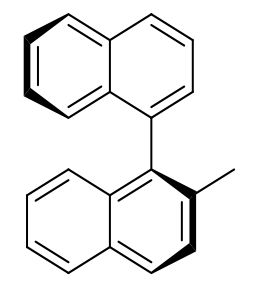

(S)-2-methyl-1,1'-binaphthalene $(1 \mathrm{~A})^{2}$

Prepared according to the general procedure, purified by flash column chromatography $(\mathrm{PE} / \mathrm{EA}=$ 100:1), white solid (2.3 g, 62\% yield). ${ }^{1} \mathbf{H} \mathbf{~ N M R}\left(\mathrm{CDCl}_{3}, 400 \mathrm{MHz}\right) \delta 7.97$ (d, J=8.4 Hz, $\left.2 \mathrm{H}\right), 7.87-7.90$ $(\mathrm{m}, 2 \mathrm{H}), 7.61(\mathrm{~d}, J=8.4 \mathrm{~Hz}, 1 \mathrm{H}), 7.50$ (t, $J=6.6 \mathrm{~Hz}, 2 \mathrm{H}), 7.39(\mathrm{~d}, J=6.8 \mathrm{~Hz}, 2 \mathrm{H}), 7.17-7.28(\mathrm{~m}, 4 \mathrm{H})$, $2.12(\mathrm{~s}, 3 \mathrm{H})$.<smiles>COc1ccc2ccccc2c1-c1c(C)ccc2ccccc12</smiles>

\section{(S)-2-methoxy-2'-methyl-1,1'-binaphthalene (1B $)^{2}$}

Prepared according to the general procedure, purified by flash column chromatography $(\mathrm{PE} / \mathrm{EA}=50: 1)$, white solid (3.0 g, 68\% yield). ${ }^{1} \mathbf{H} \mathbf{~ N M R}\left(\mathrm{CDCl}_{3}, 400 \mathrm{MHz}\right) \delta$ 7.87-8.12 (m, $\left.4 \mathrm{H}\right)$, 7.41-7.54 (m, $\left.2 \mathrm{H}\right)$, 7.24-7.39 (m, 2 H), 7.19-7.22 (m, 2 H), 7.13-7.16 (m, 1 H), 7.00-7.04 (m, 1 H), 3.80 (s, 3 H), 2.11 (s, 3 $\mathrm{H})$. 


\section{(S)-2'-methyl-[1,1'-binaphthalen]-2-yl acetate $(1 \mathrm{C})^{2}$}

Prepared according to the general procedure, purified by flash column chromatography $(\mathrm{PE} / \mathrm{EA}=50: 1)$, light yellow solid (2.5 g, 56\% yield). ${ }^{1} \mathbf{H}$ NMR $\left(\mathrm{CDCl}_{3}, 400 \mathrm{MHz}\right) \delta 7.93-8.00(\mathrm{~m}, 2 \mathrm{H}), 7.86(\mathrm{~d}, J=8.4$ Hz, 2 H), 7.36-7.48 (m, 4 H), 7.21-7.28 (m, 2 H), 2.09 (s, 3 H), 1.72 (s, 3 H).

\subsubsection{General procedure A}

$N$-hydroxyphthalimide (NHPI) (25 mg, $0.15 \mathrm{mmol}, 0.3$ equiv) and $\mathrm{AlCl}_{3}$ (20 mg, $0.15 \mathrm{mmol}, 0.3$ equiv) were weighed into a Schlenk tube. After dried in vacuo for 15 min under a argon atmosphere, MeCN (1 $\mathrm{mL}$, containing $0.1 \% \mathrm{H}_{2} \mathrm{O}$ ) was added. Then first portion of $t$-BuONO (80 $\mu \mathrm{L}$; totally $154 \mathrm{mg}, 1.5 \mathrm{mmol}$, 3.0 equiv) was added. Methylarene ( $0.5 \mathrm{mmol}, 1.0$ equiv) was last added and stirred at $80{ }^{\circ} \mathrm{C}$ for $36 \mathrm{~h}$. The rest 2 portions of $t$-BuONO was added in every 10 hours. After cooling to rt, the reaction was diluted with $\mathrm{CH}_{2} \mathrm{Cl}_{2}$ (2 $\left.\mathrm{mL}\right)$ and the resulting mixture was filtered, concentrated and purified on column chromatography to give the pure product.

\subsubsection{General procedure B}

NHPI (61 mg, $0.375 \mathrm{mmol}, 1.5$ equiv) and $\mathrm{AlCl}_{3}$ (17 mg, $0.125 \mathrm{mmol}, 0.5$ equiv) were weighed into a Schlenk tube. After dried in vacuo for 15 min under a argon atmosphere, MeCN (1 mL, containing 0.1\% $\left.\mathrm{H}_{2} \mathrm{O}\right)$ was added. Then first portion of $t$-BuONO $(80 \mu \mathrm{L}$; totally $154 \mathrm{mg}, 1.5 \mathrm{mmol}, 6.0$ equiv $)$ was added. Methylarene $\left(0.25 \mathrm{mmol}, 1.0\right.$ equiv) was last added and stirred at $90{ }^{\circ} \mathrm{C}$ for $48 \mathrm{~h}$. The rest 2 portions of $t$ BuONO was added in every 10 hours. After cooling to rt, the reaction was diluted with $\mathrm{CH}_{2} \mathrm{Cl}_{2}(2 \mathrm{~mL})$ and the resulting mixture was filtered, concentrated and purified on column chromatography to give the pure product.<smiles>Cc1ccc(C#N)cc1</smiles>

\section{4-Methylbenzonitrile $(2 a)^{3}$}

Prepared according to the general procedure A, purified by flash column chromatography $(\mathrm{PE} / \mathrm{EA}=$ 50:1), white solid (48.0 mg, 82\% yield). ${ }^{1} \mathbf{H} \mathbf{~ N M R}\left(\mathrm{CDCl}_{3}, 400 \mathrm{MHz}\right): \delta 7.54(\mathrm{~d}, J=8.0 \mathrm{~Hz}, 2 \mathrm{H}), 7.27$ $(\mathrm{d}, J=8.0 \mathrm{~Hz}, 2 \mathrm{H}), 2.42(\mathrm{~s}, 3 \mathrm{H})$. 
$\left.\right|_{C N} ^{C N}$

Prepared according to the general procedure A [NHPI (122 mg, $0.75 \mathrm{mmol}, 1.5$ equiv) and $\mathrm{AlCl}_{3}(34$ $\mathrm{mg}, 0.25 \mathrm{mmol}, 0.5$ equiv), $90^{\circ} \mathrm{C}$ ], repeat twice. purified by flash column chromatography (PE/EA=20:1), white solid (52 mg, 81\% yield). ${ }^{1} \mathbf{H}$ NMR $\left(\mathrm{CDCl}_{3}, 400 \mathrm{MHz}\right): \delta 7.80(\mathrm{~s}, 4 \mathrm{H})$.<smiles>Cc1cccc(C#N)c1</smiles>

\section{3-Methylbenzonitrile $(2 \mathbf{b})^{3}$}

Prepared according to the general procedure A, purified by flash column chromatography $(\mathrm{PE} / \mathrm{EA}=$ 50:1), light yellow oil (47.0 mg, 80\% yield). ${ }^{1} \mathbf{H} \mathbf{~ N M R}\left(\mathrm{CDCl}_{3}, 400 \mathrm{MHz}\right): \delta 7.54(\mathrm{~d}, J=8.0 \mathrm{~Hz}, 2 \mathrm{H})$, $7.27(\mathrm{~d}, J=8.0 \mathrm{~Hz}, 2 \mathrm{H}), 2.42(\mathrm{~s}, 3 \mathrm{H})$.<smiles>N#Cc1cccc(C#N)c1</smiles>

\section{Isophthalonitrile $(2 \mathbf{b A})^{5}$}

Prepared according to the general procedure A [NHPI (122 mg, $0.75 \mathrm{mmol}, 1.5$ equiv) and $\mathrm{AlCl}_{3}(34$ $\mathrm{mg}, 0.25 \mathrm{mmol}, 0.5$ equiv), $\left.90^{\circ} \mathrm{C}\right]$, repeat twice. purified by flash column chromatography $(\mathrm{PE} / \mathrm{EA}=20: 1)$, white solid (47.4 mg, 74\% yield). ${ }^{1} \mathbf{H} \mathbf{N M R}\left(\mathrm{CDCl}_{3}, 400 \mathrm{MHz}\right): \delta 7.96(\mathrm{~s}, 1 \mathrm{H}), 7.91(\mathrm{~d}, J=8.0 \mathrm{~Hz}, 2 \mathrm{H})$, $7.66(\mathrm{t}, J=7.8 \mathrm{~Hz}, 1 \mathrm{H})$.<smiles>Cc1ccccc1C#N</smiles>

\section{2-Methylbenzonitrile $(2 c)^{3}$}

Prepared according to the general procedure A, purified by flash column chromatography $(\mathrm{PE} / \mathrm{EA}=$ 50:1), colorless liquid (44.5 mg, 76\% yield). ${ }^{1} \mathbf{H} \mathbf{~ N M R}\left(\mathrm{CDCl}_{3}, 400 \mathrm{MHz}\right): \delta 7.58(\mathrm{~d}, J=8.0 \mathrm{~Hz}, 1 \mathrm{H})$, $7.48(\mathrm{t}, J=7.6 \mathrm{~Hz}, 1 \mathrm{H}), 7.31(\mathrm{~d}, J=7.6 \mathrm{~Hz}, 1 \mathrm{H}), 7.26(\mathrm{t}, J=7.6 \mathrm{~Hz}, 1 \mathrm{H}), 2.54(\mathrm{~s}, 3 \mathrm{H})$.<smiles>N#Cc1ccccc1C#N</smiles>

\section{phthalonitrile $(2 \mathrm{cA})^{6}$}

Prepared according to the general procedure A [NHPI (122 mg, $0.75 \mathrm{mmol}, 1.5$ equiv) and $\mathrm{AlCl}_{3}(34$ $\mathrm{mg}, 0.25 \mathrm{mmol}, 0.5$ equiv), $90^{\circ} \mathrm{C}$, repeat twice. purified by flash column chromatography $(\mathrm{PE} / \mathrm{EA}=20: 1)$, white solid (42.9 mg, 67\% yield). ${ }^{1} \mathbf{H}$ NMR ( $\left.\mathrm{CDCl}_{3}, 400 \mathrm{MHz}\right): \delta 7.83$ (s, $\left.2 \mathrm{H}\right), 7.78$ (s, $\left.2 \mathrm{H}\right)$.

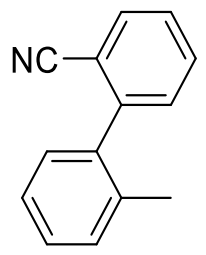


Prepared according to the general procedure A [NHPI ( $82 \mathrm{mg}, 0.5 \mathrm{mmol}, 1.0$ equiv), $\mathrm{AlCl}_{3}(34 \mathrm{mg}, 0.25$ mmol, 0.5 equiv), $90^{\circ} \mathrm{C}$ ]. purified by flash column chromatography (PE/EA $\left.=80: 1\right)$, light yellow oil (75 mg, 78\% yield). ${ }^{1} \mathbf{H}$ NMR $\left(\mathrm{CDCl}_{3}, 400 \mathrm{MHz}\right): \delta 7.67$ (d, J= 8.0 Hz, $\left.1 \mathrm{H}\right), 7.53-7.58(\mathrm{~m}, 1 \mathrm{H})$, 7.40-7.36 $(\mathrm{m}, 1 \mathrm{H}), 7.31-7.17(\mathrm{~m}, 4 \mathrm{H}), 7.13(\mathrm{~d}, J=6.8 \mathrm{~Hz}, 1 \mathrm{H}), 2.12(\mathrm{~S}, 3 \mathrm{H}) ;{ }^{13} \mathbf{C ~ N M R}\left(\mathrm{CDCl}_{3}, 100 \mathrm{MHz}\right): \delta$ 145.8, 138.0, 135.6, 132.8, 132.4, 130.4, 130.3, 129.4, 128.7, 127.5, 125.8, 118.2, 112.8, 19.8 .

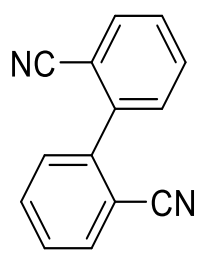

\section{$\left[1,1^{\prime}\right.$-biphenyl]-2,2'-dicarbonitrile $(2 \mathrm{dA})^{8}$}

Prepared according to the general procedure A [NHPI (122 mg, $0.75 \mathrm{mmol}, 1.5$ equiv) and $\mathrm{AlCl}_{3}(34$ $\mathrm{mg}, 0.25 \mathrm{mmol}, 0.5$ equiv), $90^{\circ} \mathrm{C}$ ], repeat twice. purified by flash column chromatography (PE/EA=10:1), white solid (75.6 mg, 74\% yield). ${ }^{1} \mathbf{H}$ NMR $\left(\mathrm{CDCl}_{3}, 400 \mathrm{MHz}\right): \delta$ 7.85-7.83 (m, $\left.2 \mathrm{H}\right)$, 7.75-7.71 (m, $\left.2 \mathrm{H}\right)$, 7.60-7.56 (m, $4 \mathrm{H})$.<smiles>Cc1ccc2ccccc2c1-c1c(C)ccc2ccccc12</smiles>

\section{(S)-2'-Methyl-[1,1'-binaphthalene]-2-carbonitrile (2e)}

Prepared according to the general procedure B, purified by flash column chromatography $(\mathrm{PE} / \mathrm{EA}=$ 80:1), white solid (54 mg, 74\% yield). ${ }^{1} \mathbf{H}$ NMR $\left(\mathrm{CDCl}_{3}, 400 \mathrm{MHz}\right): \delta 8.07-7.93(\mathrm{~m}, 4 \mathrm{H}), 7.83$ (d, $J=$ $8.8 \mathrm{~Hz}, 1 \mathrm{H}), 7.65$ (t, $J=7.4 \mathrm{~Hz}, 1 \mathrm{H}), 7.56(\mathrm{~d}, J=8.4 \mathrm{~Hz}, 1 \mathrm{H}), 7.46-7.38$ (m, $2 \mathrm{H}), 7.29-7.25$ (m, $2 \mathrm{H})$, $6.95(\mathrm{~d}, J=8.4,1 \mathrm{H}), 2.16(\mathrm{~s}, 3 \mathrm{H}) ;{ }^{13} \mathbf{C ~ N M R}\left(\mathrm{CDCl}_{3}, 100 \mathrm{MHz}\right): \delta 144.6,134.8,132.6,132.1,132.0$, $131.8,129.1,128.9,128.7,128.6,128.4,128.1,127.8,127.0,126.9,126.6,125.2,125.0,118.2,111.2$, 20.1. HRMS (ESI) calcd for $\mathrm{C}_{22} \mathrm{H}_{16} \mathrm{~N}[\mathrm{M}+\mathrm{H}]^{+} 294.1277$, found 294.1267; $[\alpha]_{\mathrm{D}}{ }^{20}+27.0^{\circ}\left(c 1.0, \mathrm{CHCl}_{3}\right)$. The enantiomeric excess was determined to be $>99.9 \%$ by HPLC analysis on Daicel OD-H column (2propanol: $n$-hexane $=5: 95(\mathrm{v}: \mathrm{v}), 0.5 \mathrm{~mL} / \mathrm{min}), \mathrm{UV} 254 \mathrm{~nm}, \mathrm{t}_{\text {minor }}=9.63 \mathrm{~min}, \mathrm{t}_{\text {major }}=8.11 \mathrm{~min}$.<smiles>N#Cc1ccc2ccccc2c1-c1c(C#N)ccc2ccccc12</smiles>

(S)-[1,1'-binaphthalene]-2,2'-dicarbonitrile $(2 \mathrm{eA})^{9}$

Prepared according to the general procedure B, repeat twice, purified by flash column chromatography $(\mathrm{PE} / \mathrm{EA}=10: 1)$, white solid $(54 \mathrm{mg}, 74 \%$ yield $) .{ }^{1} \mathbf{H} \mathbf{N M R}\left(\mathrm{CDCl}_{3}, 400 \mathrm{MHz}\right): \delta 8.13(\mathrm{~d}, J=8.4 \mathrm{~Hz}, 2$ H), $8.03(\mathrm{~d}, J=8.0 \mathrm{~Hz}, 2 \mathrm{H}), 7.85(\mathrm{~d}, J=8.4 \mathrm{~Hz}, 2 \mathrm{H}), 7.67(\mathrm{t}, J=7.6 \mathrm{~Hz}, 2 \mathrm{H}), 7.44(\mathrm{t}, J=8.2 \mathrm{~Hz}, 2 \mathrm{H})$, $7.18(\mathrm{~d}, J=8.4,2 \mathrm{H})$. The enantiomeric excess was determined to be $93 \%$ by HPLC analysis on Daicel 
OD-H column (2-propanol: $n$-hexane $=20: 80(\mathrm{v}: \mathrm{v}), 0.5 \mathrm{~mL} / \mathrm{min}), \mathrm{UV} 254 \mathrm{~nm}, \mathrm{t}_{\text {minor }}=21.65$ min, $\mathrm{t}_{\text {major }}=$ $23.91 \mathrm{~min}$.<smiles>Cc1cc2ccccc2c(-c2c(C#N)c(C)cc3ccccc23)c1C#N</smiles>

(S)-3,3'-Dimethyl-[1,1'-binaphthalene]-2,2'-dicarbonitrile (2fA)

Prepared according to the general procedure $\mathrm{B}$, purified by flash column chromatography $(\mathrm{PE} / \mathrm{EA}=$ 80:1), light yellow solid (58 mg, $70 \%$ yield). ${ }^{1} \mathbf{H} \mathbf{~ N M R}\left(\mathrm{CDCl}_{3}, 400 \mathrm{MHz}\right): \delta 8.41$ (s, 2H), 7.98 (d, $J=8.4$ $\mathrm{Hz}, 2 \mathrm{H}), 7.56(\mathrm{t}, J=7.6 \mathrm{~Hz}, 2 \mathrm{H}), 7.41(\mathrm{t}, J=7.6 \mathrm{~Hz}, 2 \mathrm{H}), 6.97(\mathrm{~d}, J=8.4 \mathrm{~Hz}, 2 \mathrm{H}), 2.20(\mathrm{~s}, 6 \mathrm{H}) ;{ }^{13} \mathrm{C}$ NMR $\left(\mathrm{CDCl}_{3}, 100 \mathrm{MHz}\right): \delta 135.4,135.1,134.2,133.9,131.1,129.9,128.8,127.1,125.4,118.4,111.9$, 18.3. HRMS (ESI) calcd for $\mathrm{C}_{24} \mathrm{H}_{16} \mathrm{~N}_{2} \mathrm{Na}[\mathrm{M}+\mathrm{Na}]^{+} 355.1211$, found 355.1205 ; $[\alpha]_{\mathrm{D}}{ }^{20}-34.0^{\circ}(c 1.0$, $\mathrm{CHCl}_{3}$ ). The enantiomeric excess was determined to be $>99.9 \%$ by HPLC analysis on Daicel OD-H column (2-propanol: $n$-hexane = 20:60 (v:v), $1.0 \mathrm{~mL} / \mathrm{min}), \mathrm{UV} 254 \mathrm{~nm}, \mathrm{t}_{\text {minor }}=19.79 \mathrm{~min}, \mathrm{t}_{\text {major }}=16.69$ $\min$.<smiles>COc1cccc(C#N)c1-c1c(C)cccc1C#N</smiles>

\section{(S)-6,6'-Dimethoxy-[1,1'-biphenyl]-2,2'-dicarbonitrile (2gA)}

Prepared according to the general procedure B, purified by flash column chromatography $(\mathrm{PE} / \mathrm{EA}=$ 50:1), white solid (52 mg, 78\% yield). ${ }^{1} \mathbf{H}$ NMR $\left(\mathrm{CDCl}_{3}, 400 \mathrm{MHz}\right): \delta$ 7.38-7.34 (m, $\left.2 \mathrm{H}\right), 7.23-7.21$ (m, $2 \mathrm{H}), 7.13-7.11(\mathrm{~m}, 2 \mathrm{H}), 3.80$ (s, $6 \mathrm{H}) ;{ }^{13} \mathbf{C ~ N M R}\left(\mathrm{CDCl}_{3}, 100 \mathrm{MHz}\right): \delta 159.6,130.2,124.4,119.2,118.7$, 116.7, 113.0, 55.4. HRMS (ESI) calcd for $\mathrm{C}_{24} \mathrm{H}_{16} \mathrm{~N}_{2} \mathrm{Na}[\mathrm{M}+\mathrm{Na}]^{+} 287.0796$, found 287.0791; $[\alpha]_{\mathrm{D}}{ }^{20}+$ $86.3^{\circ}\left(c 1.0, \mathrm{CHCl}_{3}\right)$ The enantiomeric excess was determined to be $95.4 \%$ by HPLC analysis on Daicel AD-H column (2-propanol: $n$-hexane $=10: 90(\mathrm{v}: \mathrm{v}), 1 \mathrm{~mL} / \mathrm{min}), \mathrm{UV} 210 \mathrm{~nm}, \mathrm{t}_{\text {minor }}=8.48 \mathrm{~min}$, $\mathrm{t}_{\text {major }}=6.81$ $\min$.<smiles>N#Cc1ccccc1</smiles>

\section{Benzonitrile (2h) $)^{3}$}

Prepared according to the general procedure A [NHPI ( $82 \mathrm{mg}, 0.5 \mathrm{mmol}, 1.0$ equiv) and $\mathrm{AlCl}_{3}(34 \mathrm{mg}$, $0.25 \mathrm{mmol}, 0.5$ equiv)]. 84\% yield based on GC analysis. ${ }^{1} \mathbf{H} \mathbf{~ N M R}\left(\mathrm{CDCl}_{3}, 400 \mathrm{MHz}\right): \delta 7.65-7.58(\mathrm{~m}$, $3 \mathrm{H}), 7.48-7.44$ (m, $2 \mathrm{H})$. 
OMe

4-Methoxybenzonitrile $(2 \mathrm{i})^{3}$

Prepared according to the general procedure A, purified by flash column chromatography $(\mathrm{PE} / \mathrm{EA}=$ 40:1), white solid (54 mg, 83\% yield). ${ }^{1} \mathbf{H}$ NMR $\left(\mathrm{CDCl}_{3}, 400 \mathrm{MHz}\right): \delta 7.58$ (d, J=8.0 Hz, $\left.2 \mathrm{H}\right), 6.95$ (d, $J=8.8 \mathrm{~Hz}, 2 \mathrm{H}), 3.86(\mathrm{~s}, 3 \mathrm{H})$.<smiles>N#Cc1ccc(Cl)cc1</smiles>

\section{4-Chlorobenzonitrile $(2 \mathbf{j})^{10}$}

Prepared according to the general procedure A [NHPI (41 mg, $0.25 \mathrm{mmol}, 0.5$ equiv) and $\mathrm{AlCl}_{3}(34 \mathrm{mg}$, $0.25 \mathrm{mmol}, 0.5$ equiv)], purified by flash column chromatography (PE/EA = 40:1), white solid (61 mg, 83\% yield). ${ }^{1} \mathbf{H}$ NMR $\left(\mathrm{CDCl}_{3}, 400 \mathrm{MHz}\right): \delta 7.60(\mathrm{~d}, J=8.4 \mathrm{~Hz}, 2 \mathrm{H}), 7.47(\mathrm{~d}, J=8.4 \mathrm{~Hz}, 2 \mathrm{H})$.<smiles>N#Cc1ccc(Br)cc1</smiles>

4-Bromobenzonitrile $(2 \mathbf{k})^{10}$

Prepared according to the general procedure A [NHPI ( $82 \mathrm{mg}, 0.5 \mathrm{mmol}, 1.0$ equiv) and $\mathrm{AlCl}_{3}(34 \mathrm{mg}$, $0.25 \mathrm{mmol}, 0.5$ equiv)], purified by flash column chromatography (PE/EA = 40:1), light yellow solid (76 mg, 83\% yield). ${ }^{1} \mathbf{H}$ NMR $\left(\mathrm{CDCl}_{3}, 400 \mathrm{MHz}\right): \delta$ 7.65-7.62 (m, $\left.2 \mathrm{H}\right)$, 7.54-7.52 (m, $\left.2 \mathrm{H}\right)$.<smiles>N#Cc1ccc(I)cc1</smiles>

4-Iodobenzonitrile $(2 \mathrm{l})^{3}$

Prepared according to the general procedure A [NHPI (41 mg, $0.25 \mathrm{mmol}, 0.5$ equiv) and $\mathrm{AlCl}_{3}(34 \mathrm{mg}$, $0.25 \mathrm{mmol}, 0.5$ equiv)], purified by flash column chromatography (PE/EA = 50:1), solid (92 mg, 80\% yield). ${ }^{1} \mathbf{H}$ NMR $\left(\mathrm{CDCl}_{3}, 400 \mathrm{MHz}\right): \delta$ 7.85-7.82 (m, $\left.2 \mathrm{H}\right)$, 7.37-7.35 (m, $\left.2 \mathrm{H}\right)$.<smiles>CC(F)(F)c1ccc(C#N)cc1</smiles>

\section{4-Acetylbenzonitrile $(2 \mathrm{~m})^{3}$}

Prepared according to the general procedure A [NHPI (122 mg, $0.75 \mathrm{mmol}, 1.5$ equiv) and $\mathrm{AlCl}_{3}(34$ $\mathrm{mg}, 0.25 \mathrm{mmol}, 0.5$ equiv), at $90{ }^{\circ} \mathrm{C}$ ], purified by flash column chromatography $(\mathrm{PE} / \mathrm{EA}=20: 1)$, light yellow solid (56 mg, 77\% yield). ${ }^{1} \mathbf{H} \mathbf{~ N M R}\left(\mathrm{CDCl}_{3}, 400 \mathrm{MHz}\right): \delta 8.02(\mathrm{~d}, J=8.4 \mathrm{~Hz}, 2 \mathrm{H}), 7.75(\mathrm{~d}, J=$ 
$8.0 \mathrm{~Hz}, 2 \mathrm{H}), 2.62(\mathrm{~s}, 3 \mathrm{H})$.



4-Nitrobenzonitrile $(2 n)^{3}$

Prepared according to the general procedure A [NHPI (122 mg, $0.75 \mathrm{mmol}, 1.5$ equiv) and $\mathrm{AlCl}_{3}(34$ $\mathrm{mg}, 0.25 \mathrm{mmol}, 0.5$ equiv), $90^{\circ} \mathrm{C}$ ], purified by flash column chromatography $(\mathrm{PE} / \mathrm{EA}=20: 1)$, white solid (45.1 mg, 31\% yield). ${ }^{1} \mathbf{H}$ NMR $\left(\mathrm{CDCl}_{3}, 400 \mathrm{MHz}\right): \delta 8.36(\mathrm{~d}, J=8.8 \mathrm{~Hz}, 2 \mathrm{H}), 7.89(\mathrm{~d}, J=9.2 \mathrm{~Hz}, 2 \mathrm{H})$.<smiles>N#Cc1ccc(C(F)(F)F)cc1</smiles>

\section{4-(Trifluoromethyl)benzonitrile (2o $)^{10}$}

Prepared according to the general procedure A [NHPI (122 mg, $0.75 \mathrm{mmol}, 1.5$ equiv) and $\mathrm{AlCl}_{3}(34$ $\mathrm{mg}, 0.25 \mathrm{mmol}, 0.5$ equiv), $90^{\circ} \mathrm{C}$ ], purified by flash column chromatography (PE/EA = 20:1), white solid (37.9 mg, 44\% yield). ${ }^{1} \mathbf{H} \mathbf{~ N M R}\left(\mathrm{CDCl}_{3}, 400 \mathrm{MHz}\right): \delta 7.81(\mathrm{~d}, J=8.0 \mathrm{~Hz}, 2 \mathrm{H}), 7.76(\mathrm{~d}, J=8.0 \mathrm{~Hz}, 2 \mathrm{H})$.<smiles>N#Cc1ccc2ccccc2c1</smiles>

\section{2-Naphthonitrile $(2 \mathbf{p A})^{3}$}

Prepared according to the general procedure A, purified by flash column chromatography $(\mathrm{PE} / \mathrm{EA}=$ 80:1), light yellow oil (69 mg, 90\% yield). ${ }^{1} \mathbf{H}$ NMR $\left(\mathrm{CDCl}_{3}, 400 \mathrm{MHz}\right): \delta 8.24$ (s, $\left.1 \mathrm{H}\right), 7.93-7.89$ (m, 3 H), 7.67-7.59 (m, $3 \mathrm{H})$.<smiles>N#Cc1cccc2ccccc12</smiles>

\section{1-Naphthonitrile $(2 \mathrm{pB})^{3}$}

Prepared according to the general procedure A, purified by flash column chromatography $(\mathrm{PE} / \mathrm{EA}=$ 80:1), as solid (70 mg, 92\% yield). ${ }^{1} \mathbf{H} \mathbf{~ N M R}\left(\mathrm{CDCl}_{3}, 400 \mathrm{MHz}\right): \delta 8.22$ (d, $\left.J=8.4 \mathrm{~Hz}, 1 \mathrm{H}\right), 8.06(\mathrm{~d}, J=$ 8.8 Hz, $1 \mathrm{H})$, 7.92-7.88 (m, $2 \mathrm{H})$, 7.70-7.66 (m, $1 \mathrm{H})$, 7.63-7.58 (m, $1 \mathrm{H})$, 7.53-7.49 (m, $1 \mathrm{H})$.<smiles>N#Cc1c2ccccc2cc2ccccc12</smiles>

\section{9-Anthronitrile $(2 q)^{3}$}

Prepared according to the general procedure A, purified by flash column chromatography $(\mathrm{PE} / \mathrm{EA}=$ 100:1), as yellow solid (84 mg, 82\% yield). ${ }^{1} \mathbf{H} \mathbf{~ N M R}\left(\mathrm{CDCl}_{3}, 400 \mathrm{MHz}\right): \delta 8.70(\mathrm{~s}, 1 \mathrm{H}), 8.44(\mathrm{~d}, J=8.0$ Hz, 2 H), 8.09 (d, J=8.4 Hz, 2 H), 7.73 (t, $J=7.2 \mathrm{~Hz}, 2 \mathrm{H}), 7.60$ (t, $J=7.6 \mathrm{~Hz}, 2 \mathrm{H}$ ). 


\section{2-Phenylbenzonitrile (2r) $)^{12}$}

Prepared according to the general procedure A [NHPI ( $82 \mathrm{mg}, 0.5 \mathrm{mmol}, 1.0$ equiv), $\mathrm{AlCl}_{3}$ ( $67 \mathrm{mg}, 0.5$ mmol, 1.0 equiv), $90^{\circ} \mathrm{C}$ ], purified by flash column chromatography (PE/EA $\left.=80: 1\right)$, light yellow oil $(76$ mg, 82\% yield) ${ }^{1} \mathbf{H}$ NMR $\left(\mathrm{CDCl}_{3}, 400 \mathrm{MHz}\right): \delta 7.77(\mathrm{~d}, J=8.0 \mathrm{~Hz}, 1 \mathrm{H}), 7.67-7.63(\mathrm{~m}, 1 \mathrm{H}), 7.59-7.54$ (m, $2 \mathrm{H}), 7.52-7.43$ (m, $5 \mathrm{H}) ;{ }^{13} \mathrm{C}$ NMR (CDCl $\left.3,100 \mathrm{MHz}\right): \delta$ 145.4, 138.0, 133.7, 132.7, 130.0, 128.7, 128.6, 127.5, 118.6, 111.2.<smiles>Cn1cc(C#N)c2ccccc21</smiles>

\section{1-Acetyl-1H-indole-3-carbonitrile $(2 \mathrm{~s})^{3}$}

Prepared according to the general procedure A [NHPI ( $82 \mathrm{mg}, 0.5 \mathrm{mmol}, 1.0$ equiv), $\mathrm{AlCl}_{3}$ ( $34 \mathrm{mg}, 0.25$ mmol, 0.5 equiv), with $\mathrm{K}_{2} \mathrm{CO}_{3}\left(52 \mathrm{mg}, 0.375 \mathrm{mmol}, 0.75\right.$ equiv), $90{ }^{\circ} \mathrm{C}$ ], purified by flash column chromatography (PE/EA = 10:1), white solid (50.5 mg, 61\% yield). ${ }^{1} \mathbf{H}$ NMR $\left(\mathrm{CDCl}_{3}, 400 \mathrm{MHz}\right): \delta 8.44$ (d, $J=8.4 \mathrm{~Hz}, 1 \mathrm{H}), 7.98$ (s, $1 \mathrm{H}), 7.74(\mathrm{~d}, J=7.6 \mathrm{~Hz}, 1 \mathrm{H}), 7.51-7.42(\mathrm{~m}, 2 \mathrm{H}), 2.71$ (s, $3 \mathrm{H})$.<smiles>N#Cc1cccnc1</smiles>

\section{Nicotinonitrile $(2 \mathrm{tA})^{11}$}

Prepared according to the general procedure A [NHPI ( $82 \mathrm{mg}, 0.5 \mathrm{mmol}, 1.0$ equiv), $\mathrm{AlCl}_{3}$ ( $34 \mathrm{mg}, 0.25$ mmol, 0.5 equiv), with $\mathrm{K}_{2} \mathrm{CO}_{3}$ (52 $\mathrm{mg}, 0.375 \mathrm{mmol}, 0.75$ equiv), $90{ }^{\circ} \mathrm{C}$ ], purified by flash column chromatography (PE/EA = 5:1), white solid (56 mg, 43\% yield). ${ }^{1} \mathbf{H}$ NMR $\left(\mathrm{CDCl}_{3}, 400 \mathrm{MHz}\right): \delta 8.90$ 8.80 (m, 2 H), 7.98-7.95 (m, 1 H), 7.45-7.42 (m, 1 H).<smiles>Cc1cccc(C#N)n1</smiles>

\section{6-Methylpicolinonitrile $(2 \mathrm{tB})^{3}$}

Prepared according to the general procedure A [NHPI ( $82 \mathrm{mg}, 0.5 \mathrm{mmol}, 1.0$ equiv), $\mathrm{AlCl}_{3}$ ( $34 \mathrm{mg}, 0.25$ mmol, 0.5 equiv), with $\mathrm{K}_{2} \mathrm{CO}_{3}$ (52 $\mathrm{mg}, 0.375 \mathrm{mmol}, 0.75$ equiv), $90{ }^{\circ} \mathrm{C}$ ], purified by flash column chromatography (PE/EA = 5:1), white solid (22 mg, 37\% yield). ${ }^{1} \mathbf{H}$ NMR $\left(\mathrm{CDCl}_{3}, 400 \mathrm{MHz}\right): \delta 7.71$ (t, $J=7.6 \mathrm{~Hz}, 1 \mathrm{H}), 7.52(\mathrm{~d}, J=7.6 \mathrm{~Hz}, 1 \mathrm{H}), 7.37$ (d, $J=7.6 \mathrm{~Hz}, 1 \mathrm{H}), 2.61(\mathrm{~s}, 3 \mathrm{H})$.

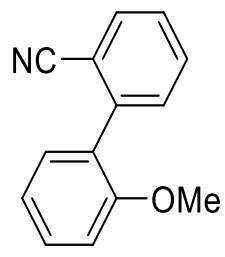

\section{2'-Methoxy-[1,1'-biphenyl]-2-carbonitrile (2u) $)^{7}$}

Prepared according to the general procedure A [NHPI ( $82 \mathrm{mg}, 0.5 \mathrm{mmol}, 1.0$ equiv), $\mathrm{AlCl}_{3}$ ( $34 \mathrm{mg}, 0.25$ 
mmol, 0.5 equiv), $90^{\circ} \mathrm{C}$ ], purified by flash column chromatography (PE/EA = 80:1), light yellow oil (84 mg, 80\% yield). ${ }^{1} \mathbf{H}$ NMR $\left(\mathrm{CDCl}_{3}, 400 \mathrm{MHz}\right): \delta 7.72$ (d, $\left.J=7.6 \mathrm{~Hz}, 1 \mathrm{H}\right), 7.63-7.60$ (m, $\left.1 \mathrm{H}\right)$, 7.46-7.39 $(\mathrm{m}, 3 \mathrm{H}), 7.27-7.24(\mathrm{~m}, 1 \mathrm{H}), 7.07-7.01(\mathrm{~m}, 2 \mathrm{H}), 3.84(\mathrm{~s}, 3 \mathrm{H}) ;{ }^{13} \mathbf{C ~ N M R}\left(\mathrm{CDCl}_{3}, 100 \mathrm{MHz}\right): \delta 156.4$, $142.5,132.7,132.4,130.9,130.8,127.3,127.2,120.7,118.6,113.4,111.2,55.4$.

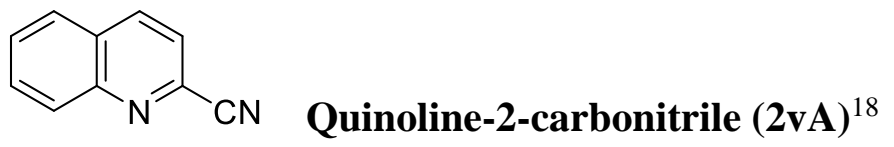

Prepared according to the general procedure A [NHPI ( $82 \mathrm{mg}, 0.5 \mathrm{mmol}, 1.0$ equiv), $\mathrm{AlCl}_{3}(34 \mathrm{mg}, 0.25$ mmol, 0.5 equiv), with $\mathrm{K}_{2} \mathrm{CO}_{3}\left(52 \mathrm{mg}, 0.375 \mathrm{mmol}, 0.75\right.$ equiv), $90{ }^{\circ} \mathrm{C}$ ], purified by flash column chromatography $(\mathrm{PE} / \mathrm{EA}=10: 1)$, white solid $(41 \mathrm{mg}, 53 \%$ yield $) .{ }^{1} \mathbf{H} \mathbf{~ N M R}\left(\mathrm{CDCl}_{3}, 400 \mathrm{MHz}\right): \delta 8.06(\mathrm{~d}$, $J=8.0 \mathrm{~Hz}, 1 \mathrm{H}), 7.99(\mathrm{~d}, J=8.4 \mathrm{~Hz}, 1 \mathrm{H}), 7.80(\mathrm{~d}, J=8.4 \mathrm{~Hz}, 1 \mathrm{H}) 7.73(\mathrm{t}, J=7.8 \mathrm{~Hz}, 1 \mathrm{H}), 7.57(\mathrm{t}, J=$ $7.0 \mathrm{~Hz}, 1 \mathrm{H}), 7.51(\mathrm{~d}, J=8.4 \mathrm{~Hz}, 1 \mathrm{H})$.<smiles>N#Cc1ccnc2ccccc12</smiles>

\section{Quinoline-4-carbonitrile (2vB) ${ }^{18}$}

Prepared according to the general procedure A [NHPI ( $82 \mathrm{mg}, 0.5 \mathrm{mmol}, 1.0$ equiv), $\mathrm{AlCl}_{3}$ (34 mg, 0.25 mmol, 0.5 equiv), with $\mathrm{K}_{2} \mathrm{CO}_{3}\left(52 \mathrm{mg}, 0.375 \mathrm{mmol}, 0.75\right.$ equiv), $90{ }^{\circ} \mathrm{C}$ ], purified by flash column chromatography $(\mathrm{PE} / \mathrm{EA}=10: 1)$, white solid (39.3 mg, 51\% yield). ${ }^{1} \mathbf{H} \mathbf{~ N M R}\left(\mathrm{CDCl}_{3}, 400 \mathrm{MHz}\right): \delta 8.78$ $(\mathrm{d}, J=4.8 \mathrm{~Hz}, 1 \mathrm{H}), 8.23(\mathrm{~d}, J=8.4 \mathrm{~Hz}, 1 \mathrm{H}), 8.14(\mathrm{~d}, J=8.4 \mathrm{~Hz}, 1 \mathrm{H}) 7.78(\mathrm{t}, J=7.4 \mathrm{~Hz}, 1 \mathrm{H}), 7.65(\mathrm{t}$, $J=7.8 \mathrm{~Hz}, 1 \mathrm{H}), 7.50(\mathrm{~d}, J=4.8 \mathrm{~Hz}, 1 \mathrm{H})$.<smiles>N#Cc1cncc2ccccc12</smiles>

\section{Isoquinoline-4-carbonitrile $(2 \mathrm{vC})^{19}$}

Prepared according to the general procedure A [NHPI ( $82 \mathrm{mg}, 0.5 \mathrm{mmol}, 1.0$ equiv), $\mathrm{AlCl}_{3}(34 \mathrm{mg}, 0.25$ mmol, 0.5 equiv), with $\mathrm{K}_{2} \mathrm{CO}_{3}\left(52 \mathrm{mg}, 0.375 \mathrm{mmol}, 0.75\right.$ equiv), $90{ }^{\circ} \mathrm{C}$ ], purified by flash column chromatography $(\mathrm{PE} / \mathrm{EA}=10: 1)$, white solid $\left(35.4 \mathrm{mg}, 46 \%\right.$ yield). ${ }^{1} \mathbf{H} \mathbf{~ N M R}\left(\mathrm{CDCl}_{3}, 400 \mathrm{MHz}\right): \delta 9.16$ $(\mathrm{s}, 1 \mathrm{H}), 8.72(\mathrm{~s}, 1 \mathrm{H}), 8.15(\mathrm{~d}, J=8.4 \mathrm{~Hz}, 1 \mathrm{H}), 7.97(\mathrm{~d}, J=8.4 \mathrm{~Hz}, 1 \mathrm{H}) 7.81(\mathrm{t}, J=8.8 \mathrm{~Hz}, 1 \mathrm{H}), 7.68(\mathrm{t}$, $J=8.0 \mathrm{~Hz}, 1 \mathrm{H})$.<smiles>N#Cc1cc2ccccc2o1</smiles>

\section{Benzofuran-2-carbonitrile $(\mathbf{2 w})^{19}$}

Prepared according to the general procedure A [NHPI ( $82 \mathrm{mg}, 0.5 \mathrm{mmol}, 1.0$ equiv), $\mathrm{AlCl}_{3}(34 \mathrm{mg}, 0.25$ mmol, 0.5 equiv), $90{ }^{\circ} \mathrm{C}$ ], purified by flash column chromatography (PE/EA = 80:1), yellow oil (15.1 mg, 21\% yield). ${ }^{1} \mathbf{H}$ NMR (400 MHz, $\left.\mathrm{CDCl}_{3}\right) \delta$ 7.87-7.63 (m, $\left.2 \mathrm{H}\right), 7.59-7.57$ (m, $\left.2 \mathrm{H}\right), 7.30(\mathrm{~s}, 1 \mathrm{H})$. 


\section{Thiophene-2-carbonitrile $(2 x)^{3}$}

Prepared according to the general procedure A [NHPI ( $82 \mathrm{mg}, 0.5 \mathrm{mmol}, 1.0$ equiv), $\mathrm{AlCl}_{3}(34 \mathrm{mg}, 0.25$ mmol, 0.5 equiv), $90^{\circ} \mathrm{C}$ ], purified by flash column chromatography (PE/EA = 10:1), yellow solid (42 mg, $39 \%$ yield). ${ }^{1} \mathbf{H}$ NMR $\left(400 \mathrm{MHz}, \mathrm{CDCl}_{3}\right) \delta$ 7.63-7.60 (m, $\left.2 \mathrm{H}\right), 7.13(\mathrm{dd}, J=5.0,3.8 \mathrm{~Hz}, 1 \mathrm{H})$.<smiles>N#Cc1nc2ccccc2s1</smiles>

\section{2-Benzothiazolecarbonitrile $(2 \mathbf{y})^{20}$}

Prepared according to the general procedure A [NHPI ( $82 \mathrm{mg}, 0.5 \mathrm{mmol}, 1.0$ equiv), $\mathrm{AlCl}_{3}(34 \mathrm{mg}, 0.25$ mmol, 0.5 equiv), $90^{\circ} \mathrm{C}$ ], purified by flash column chromatography (PE/EA = 10:1), yellow solid (25 mg, $31 \%$ yield). ${ }^{1} \mathbf{H}$ NMR (400 MHz, $\left.\mathrm{CDCl}_{3}\right) \delta 7.47$ (d, $\left.J=7.6 \mathrm{~Hz}, 1 \mathrm{H}\right) .7 .39-7.33$ (m, $\left.2 \mathrm{H}\right)$, 7.30-7.27 (m, 1 $\mathrm{H})$.<smiles>N#Cc1ccc2ccccc2c1-c1cccc2ccccc12</smiles>

\section{(S)-[1,1'-Binaphthalene]-2-carbonitrile (2A)}

Prepared according to the general procedure $\mathrm{B}$, purified by flash column chromatography $(\mathrm{PE} / \mathrm{EA}=$ 80:1), white solid (53 mg, 76\% yield). ${ }^{1} \mathbf{H}$ NMR $\left(\mathrm{CDCl}_{3}, 400 \mathrm{MHz}\right): \delta$ 8.05-7.97 (m, $\left.4 \mathrm{H}\right), 7.79$ (d, $J=$ 8.8 Hz, $1 \mathrm{H}), 7.68-7.60$ (m, $2 \mathrm{H}), 7.54-7.49$ (m, $2 \mathrm{H}), 7.41-7.26$ (m, $3 \mathrm{H}), 7.15(\mathrm{~d}, J=8.4 \mathrm{~Hz}, 1 \mathrm{H}) ;{ }^{13} \mathrm{C}$ NMR $\left(\mathrm{CDCl}_{3}, 100 \mathrm{MHz}\right): \delta 145.0,134.7,134.1,133.6,132.4,132.2,129.5,128.9,128.8,128.5,128.2$, 128.1, 127.7, 127.6, 126.8, 126.7, 126.2, 125.5, 125.4, 118.5, 111.1. HRMS (ESI) calcd for $\mathrm{C}_{21} \mathrm{H}_{13} \mathrm{NNa}$ $[\mathrm{M}+\mathrm{Na}]^{+} 302.0946$ found $302.0946 ;[\alpha]_{\mathrm{D}}^{20}+89.0^{\circ}\left(c\right.$ 1.0, $\left.\mathrm{CHCl}_{3}\right)$. The enantiomeric excess was determined to be $99.1 \%$ by HPLC analysis on Daicel OD-H column (2-propanol: $n$-hexane = 10:90 (v:v), $0.5 \mathrm{~mL} / \mathrm{min}$ ), UV $254 \mathrm{~nm}, \mathrm{t}_{\text {minor }}=41.41 \mathrm{~min}, \mathrm{t}_{\mathrm{major}}=43.55 \mathrm{~min}$.<smiles>COc1ccc2ccccc2c1-c1c(C#N)ccc2ccccc12</smiles>

(S)-2'-Methoxy-[1,1'-binaphthalene]-2-carbonitrile (2B)

Prepared according to the general procedure $\mathrm{B}$, purified by flash column chromatography $(\mathrm{PE} / \mathrm{EA}=$ 80:1), white solid (55 mg, 72\% yield). ${ }^{1} \mathbf{H}$ NMR $\left(\mathrm{CDCl}_{3}, 400 \mathrm{MHz}\right): \delta 8.04$ (d, $\left.J=9.2 \mathrm{~Hz}, 1 \mathrm{H}\right), 7.80$ $7.94(\mathrm{~m}, 2 \mathrm{H}), 7.87(\mathrm{~d}, J=8.0 \mathrm{~Hz} 1 \mathrm{H}), 7.77(\mathrm{~d}, J=8.8 \mathrm{~Hz} 1 \mathrm{H}), 7.60-7.56(\mathrm{~m}, 1 \mathrm{H}), 7.46(\mathrm{~d}, J=9.2 \mathrm{~Hz}$ $1 \mathrm{H}), 7.34-7.31(\mathrm{~m}, 3 \mathrm{H}), 7.25-7.21(\mathrm{~m}, 1 \mathrm{H}), 6.90(\mathrm{~d}, J=8.4 \mathrm{~Hz}, 1 \mathrm{H}), 3.82(\mathrm{~s}, 3 \mathrm{H}) ;{ }^{13} \mathbf{C ~ N M R}\left(\mathrm{CDCl}_{3}\right.$, $100 \mathrm{MHz}): \delta 154.8,141.8,134.8,133.4,132.4,131.1,128.9,128.6,128.3,128.2,127.5,127.2,127.1$, 
126.9, 124.3, 123.8, 118.7, 118.6, 113.3, 111.8, 56.5. HRMS (ESI) calcd for $\mathrm{C}_{22} \mathrm{H}_{15} \mathrm{NONa}[\mathrm{M}+\mathrm{Na}]^{+}$ 332.1046, found 332.1037; $[\alpha]_{\mathrm{D}}^{20}+101.0^{\circ}\left(c\right.$ 1.0, $\left.\mathrm{CHCl}_{3}\right)$. The enantiomeric excess was determined to be $>99.9 \%$ by HPLC analysis on Daicel OJ-H column (2-propanol: $n$-hexane $=10: 90(\mathrm{v}: \mathrm{v}), 1.0 \mathrm{~mL} / \mathrm{min})$, UV $254 \mathrm{~nm}, \mathrm{t}_{\text {minor }}=24.24 \mathrm{~min}, \mathrm{t}_{\text {major }}=32.53 \mathrm{~min}$.<smiles>CC(=O)Oc1ccc2ccccc2c1-c1c(C#N)ccc2ccccc12</smiles>

\section{(S)-2'-Cyano-[1,1'-binaphthalen]-2-yl acetate (2C)}

Prepared according to the general procedure B, purified by flash column chromatography $(\mathrm{PE} / \mathrm{EA}=$ 80:1), light yellow solid (61 mg, 73\% yield). ${ }^{1} \mathbf{H} \mathbf{~ N M R}\left(\mathrm{CDCl}_{3}, 400 \mathrm{MHz}\right): \delta 8.10-8.03$ (m, $\left.2 \mathrm{H}\right), 7.98$ (d, $J=8.0 \mathrm{~Hz}, 2 \mathrm{H}), 7.80$ (d, $J=8.4 \mathrm{~Hz} 1 \mathrm{H}), 7.64-7.60$ (m, $1 \mathrm{H})$, 7.51-7.47 (m, $2 \mathrm{H})$, 7.42-7.26 (m, $3 \mathrm{H})$, $7.06(\mathrm{~d}, J=8.8 \mathrm{~Hz}, 1 \mathrm{H}), 1.80(\mathrm{~s}, 3 \mathrm{H}) ;{ }^{13} \mathbf{C ~ N M R}\left(\mathrm{CDCl}_{3}, 100 \mathrm{MHz}\right): \delta 168.8,146.7,139.7,134.6,132.9$, $131.9,131.6,130.8,129.2$, 129.0, 128.4, 128.2, 127.8, 127.3, 126.6, 126.0, 125.2, 124.7, 123.9, 121.8, 118.0, 111.6, 20.4. HRMS (ESI) calcd for $\mathrm{C}_{23} \mathrm{H}_{15} \mathrm{NO}_{2} \mathrm{Na}[\mathrm{M}+\mathrm{Na}]^{+} 360.0995$, found 360.0985; $[\alpha]_{\mathrm{D}}{ }^{20}+$ $52.0^{\circ}\left(c 1.0, \mathrm{CHCl}_{3}\right)$. The enantiomeric excess was determined to be $99.7 \%$ by HPLC analysis on Daicel AD-H column (2-propanol: $n$-hexane $=10: 90(\mathrm{v}: \mathrm{v}), 1.0 \mathrm{~mL} / \mathrm{min}), \mathrm{UV} 254 \mathrm{~nm}, \mathrm{t}_{\text {minor }}=15.49$ min, $\mathrm{t}_{\text {major }}=$ $12.17 \mathrm{~min}$.

\subsubsection{Procedure for the total synthesis of $( \pm)$-Citalopram}<smiles>Cc1cccc(C#N)c1</smiles>

\section{3-Methylbenzonitrile $(2 \mathrm{~b})^{3}$}

Prepared according to the general procedure A, purified by flash column chromatography $(\mathrm{PE} / \mathrm{EA}=$ $50: 1)$ to give $\mathbf{2 b}(3.26 \mathrm{~g}, 83 \%)$ as a light yellow oil.<smiles>N#Cc1cccc(CO)c1</smiles>

\section{3-cyanobenzyl alcohol (4) ${ }^{13}$}

To a $250 \mathrm{~mL}$ flask was added $m$-tolunitrile (3.26 g, $27.8 \mathrm{mmol})$ dissolved in distilled $\mathrm{CCl}_{4}(60 \mathrm{~mL})$. To this solution was added $N$-bromosuccinimide $(24.7 \mathrm{~g}, 139.0 \mathrm{mmol})$ and benzoyl peroxide (449 $\mathrm{mg}, 1.39$ $\mathrm{mmol}$ ). The reaction was refluxed for $10 \mathrm{~h}$. After cooling down to r.t., filtration and removal of the solvent under vacuum afforded a residue which was then taken up in $\mathrm{CH}_{2} \mathrm{Cl}_{2}(50 \mathrm{~mL})$ and $\mathrm{H}_{2} \mathrm{O}(50 \mathrm{ml})$. The aqueous phase was separated and further extracted with $\mathrm{CH}_{2} \mathrm{Cl}_{2}(3 \times 30 \mathrm{~mL})$. The combined organic layers 
were washed with a saturated aqueous $\mathrm{Na}_{2} \mathrm{CO}_{3}$ solution $(2 \times 20 \mathrm{~mL})$ and then with water $(2 \times 20 \mathrm{~mL})$. The organic phase was dried over $\mathrm{Na}_{2} \mathrm{SO}_{4}$, filtered and the solvent was removed under vacuum to provide crude 3-(dibromomethyl)benzonitrile.

To a solution of this crude product 3-(dibromomethyl)benzonitrile in THF (40 mL) was added $\mathrm{AgNO}_{3}$ $(18.9 \mathrm{~g}, 111.2 \mathrm{mmol})$ and $20 \mathrm{~mL}$ of water, and the mixture was heated at reflux under argon for $5.5 \mathrm{~h}$. The solution was allowed to cool to room temperature, to which was added saturated $\mathrm{NaCl}$ solution. $\mathrm{Ag}$ salt precipitate was filtered off and the filtrate was extracted with $\mathrm{Et}_{2} \mathrm{O}(3 \times 40 \mathrm{~mL})$, and the combined organic phase was washed with brine and dried over $\mathrm{Na}_{2} \mathrm{SO}_{4}$. The solvent was evaporated and got crude product 3-formylbenzonitrile.

The crude product 3-formylbenzonitrile was dissolved in $\mathrm{MeOH}(50 \mathrm{~mL})$ and $\mathrm{NaBH}_{4}(2.27 \mathrm{~g}, 60.0 \mathrm{mmol})$ was added into this solution with portionwise at $0{ }^{\circ} \mathrm{C}$. The mixture was stirred for $4 \mathrm{~h}$ at r.t. After the solution was completed, to which was added saturated $\mathrm{NH}_{4} \mathrm{Cl}$ solution, extracted with $\mathrm{Et}_{2} \mathrm{O}(3 \times 40 \mathrm{~mL})$, and the combined organic phase was washed with brine and dried over $\mathrm{Na}_{2} \mathrm{SO}_{4}$. The solvent was evaporated and the residue was purified by silica gel column chromatography $(\mathrm{PE}: \mathrm{EA}=10: 1)$ to give 3cyanobenzyl alcohol (56\%, 2.08 g, 3 steps) as a slightly yellow liquid. ${ }^{1} \mathbf{H}$ NMR ( $\left.\mathrm{CDCl}_{3}, 400 \mathrm{MHz}\right): \delta$ $7.65(\mathrm{~s}, 1 \mathrm{H}), 7.57(\mathrm{t}, J=7.6 \mathrm{~Hz}, 2 \mathrm{H}), 7.45(\mathrm{t}, J=8.0 \mathrm{~Hz}, 1 \mathrm{H}), 4.71(\mathrm{~s}, 2 \mathrm{H}), 2.44(\mathrm{~s}, 1 \mathrm{H})$.

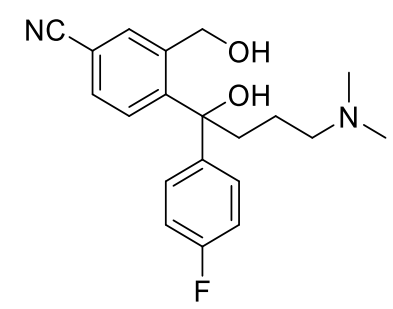

\section{4-(4-(dimethylamino)-1-(4-fluorophenyl)-1-hydroxybutyl)-3-(hydroxymethyl)benzonitril (6) ${ }^{14}$}

Into a Schlenk tube charged with 4 (2.08 g, $15.6 \mathrm{mmol}, 1.0$ equiv) was added dry THF $10 \mathrm{~mL}$ and dry TMEDA (7 mL, $46.8 \mathrm{mmol}, 3.0$ equiv). Then $t$-BuLi (21 mL, 1.6 M, $34.3 \mathrm{mmol})$ was added dropwise at $-78{ }^{\circ} \mathrm{C}$. After addition, the mixture was stirred $20 \mathrm{~min}$, then 5 (1.63 g, $\left.7.8 \mathrm{mmol}, 0.5 \mathrm{equiv}\right)$ was added. When the reaction was slowly returned to room temperature, quenched by sat. $\mathrm{NH}_{4} \mathrm{Cl}$ aqueous, the aqueous layer was extracted by EtOAc $(30 \mathrm{~mL} \times 4)$, then the organic layers was combined, washed with sat. $\mathrm{NaCl}$ aqueous, dried over anhydrous sodium sulfate, concentrated by rotary evaporation under reduced pressure and purified by flash column chromatography (DCM/MeOH =10:1) to give a solid (1.12 g, 42\%). ${ }^{1} \mathbf{H}$ NMR $\left(\mathrm{CDCl}_{3}, 400 \mathrm{MHz}\right): \delta 7.42(\mathrm{~d}, J=7.6 \mathrm{~Hz}, 2 \mathrm{H}), 7.40-7.26(\mathrm{~m}, 3 \mathrm{H}), 6.93(\mathrm{t}, J=8.6 \mathrm{~Hz}$, $2 \mathrm{H}), 4.31(\mathrm{~d}, J=12.0 \mathrm{~Hz}, 1 \mathrm{H}), 4.05(\mathrm{~d}, J=12.4 \mathrm{~Hz}, 1 \mathrm{H}), 2.46-2.42$ (m, $1 \mathrm{H}), 2.41-2.38$ (m, $2 \mathrm{H}), 2.31-$ $2.27(\mathrm{~m}, 1 \mathrm{H}), 2.42(\mathrm{~s}, 6 \mathrm{H}), 1.65-1.57(\mathrm{~m}, 1 \mathrm{H}), 1.56-1.53(\mathrm{~m}, 1 \mathrm{H}) .{ }^{13} \mathbf{C ~ N M R}\left(\mathrm{CDCl}_{3}, 100 \mathrm{MHz}\right): \delta 162.6$, 160.2 , 144.8, 143.5, 143.4, 142.9, 135.3, 129.9, 128.1, 127.6, 127.5, 121.2, 114.7, 114.4, 64.2, 59.8, 45.8, $44.6,43.7,22.0$. 


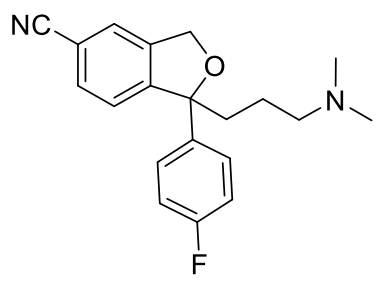

(士)-Citalopram $(7)^{15}$

Into a flask charged with $6(1.12 \mathrm{~g}, 3.3 \mathrm{mmol})$ was added $20 \mathrm{~mL}$ THF, then $60 \% \mathrm{H}_{2} \mathrm{SO}_{4}$ aqueous $10 \mathrm{~mL}$ was added slowly with cooling by ice-bath. The mixture was stirred for $5 \mathrm{~h}$ at room temperature.and quenched by sat. $\mathrm{NaHCO}_{3}$, extracted by EtOAc $(30 \mathrm{~mL} \times 4)$. The organic layers were combined, washed with sat. $\mathrm{NaCl}$ aqueous, dried over anhydrous sodium sulfate, concentrated by rotary evaporation under reduced pressure and purified by flash column chromatography (DCM/MeOH $=10: 1)$ to give a brown oil (1.02 g, 95\%). ${ }^{1} \mathbf{H}$ NMR $\left(\mathrm{CDCl}_{3}, 400 \mathrm{MHz}\right): \delta$ 7.43-7.37 (m, $\left.3 \mathrm{H}\right), 7.32$ (s, $\left.1 \mathrm{H}\right), 7.13(\mathrm{~d}, J=8.0 \mathrm{~Hz}, 1 \mathrm{H})$, $6.97(\mathrm{t}, J=8.6 \mathrm{~Hz}, 2 \mathrm{H}), 5.10(\mathrm{q}, J=12.8 \mathrm{~Hz}, 2 \mathrm{H}), 2.41(\mathrm{~s}, 1 \mathrm{H}), 2.27(\mathrm{~s}, 1 \mathrm{H}), 2.21(\mathrm{t}, J=7.2 \mathrm{~Hz}, 2 \mathrm{H})$, $2.12(\mathrm{~s}, 6 \mathrm{H}), 1.49-1.42(\mathrm{~m}, 1 \mathrm{H}), 1.35-1.25(\mathrm{~m}, 1 \mathrm{H})$.

\subsubsection{Procedure for control experiment}

NHPI (25 mg, $0.15 \mathrm{mmol}, 0.3$ equiv) was weighed into a Schlenk tube. After dried in vacuo for $15 \mathrm{~min}$ under a argon atmosphere, $\mathrm{MeCN}\left(1 \mathrm{~mL}\right.$, containing $\left.0.1 \% \mathrm{H}_{2} \mathrm{O}\right)$ was added. Then first portion of $t$-BuONO (80 $\mu \mathrm{L}$; totally $154 \mathrm{mg}, 1.5 \mathrm{mmol}, 3.0$ equiv) was added. $p$-Xylene (53 mg, $0.5 \mathrm{mmol}, 1.0$ equiv) was last added and stirred at $80{ }^{\circ} \mathrm{C}$ for $36 \mathrm{~h}$. The rest 2 portions of $t$-BuONO was added in every 10 hours. After cooling to $\mathrm{rt}$, the reaction was diluted with $\mathrm{CH}_{2} \mathrm{Cl}_{2}(2 \mathrm{~mL})$ and the resulting mixture was filtered, concentrated, then determined by ${ }^{1} \mathrm{H}$ NMR.

4-Methylbenzaldehyde oxime (68 mg, $0.5 \mathrm{mmol}, 1.0$ equiv) and $\mathrm{AlCl}_{3}(20 \mathrm{mg}, 0.15 \mathrm{mmol}, 0.3$ equiv) were weighed into a Schlenk tube. After dried in vасио for 15 min under a argon atmosphere, MeCN (1 $\mathrm{mL}$, containing $0.1 \% \mathrm{H}_{2} \mathrm{O}$ ) was added and stirred at $80{ }^{\circ} \mathrm{C}$ for $5 \mathrm{~h}$. After cooling to rt, the reaction was diluted with $\mathrm{CH}_{2} \mathrm{Cl}_{2}(2 \mathrm{~mL})$ and the resulting mixture was filtered, concentrated, then determined by ${ }^{1} \mathrm{H}$ NMR. 


\section{References}

(1) Meng, G.; Yang, T.; Liu, Y. Org. Prep. Proced. Int. 2011, 43, 354.

(2) Mosquera, Á.; Pena, M. A.; Sestelo, P. J.; Sarandeses, L. A. Eur. J. Org Chem. 2013, 2555.

(3) Shu, Z.; Ye, Y.; Deng, Y.; Zhang, Y.; Wang, J. Angew. Chem., Int. Ed. 2013, 52, 10573.

(4) Ganapathy, D.; Kotha, S. S.; Sekar, G. Tetrahedron Lett. 2015, 56, 175.

(5) Cohen, D. T.; Buchwald, S. L. Org. Lett. 2015, 17, 202.

(6) Laulhe, S.; Gori, S. S.; Nantz, M. H. J. Org. Chem. 2012, 77, 9334.

(7) Anbarasan, P.; Neumann, H.; Beller, M. Chem. Eur. J. 2010, 16, 4725.

(8) Toummini, D.; Ouazzani, F.; Taillefer, M. Org. Lett. 2015, 13, 4690.

(9) Vondenhof, M.; Mattay, J. Chem. Ber. 1990, 123, 2457.

(10) Lambert, K. M.; Bobbitt, J. M.; Eldirany, S. A.; Wiberg, K. B.; Bailey, W. F. Org. Lett. 2014, 16, 6484.

(11) Kim, J.; Choi, J.; Shin, K.; Chang, S. J. Am. Chem. Soc. 2012, 134, 2528.

(12) Zhou, C.; Liu, Q.-J.; Li, Y.-M.; Zhang, R.; Fu, X,-M.; Duan, C.-Y. J. Org. Chem. 2012, 77, 10468.

(13) Lee, J.-Y.; Ryu, T.-Y.; Park, S.-J.; Lee, P. H. J. Org. Chem. 2012, 77, 4821.

(14) Zong, H.; Huang, H.-Y.; Liu, J.-F.; Bian, G.-L.; Song, L. J. Org. Chem. 2012, 77, 4645.

(15) Mid, A.; Kumar, R.; Ramachandran, U. Org. Prep. Proced. Int. 2006, 38, 423.

(16) Holzwarth, R.; Bartsch, R.; Cherkaoui, Z.; Solladié, G. Eur. J. Org. Chem. 2005, 3536.

(17) Wen, J.-F.; Hong, W.; Yuan, K.; Mak, T. C. W.; Wong, H. N. C. J. Org. Chem. 2003, 68, 8918.

(18) Guo, S.; Wan, G.; Sun, S.; Jiang, Y.; Yu, J.-T.; Cheng, J. Chem.Comm. 2015, 51, 5085.

(19) Zhu, Y.-M.; Li, L.-Y.; Shen, Z.-M. Chem. Eur. J. 2015, 21, 13246.

(20) DO, H.-Q.; Daugulis, O. Org. Lett. 2010, 12, 2517. 
4. NMR spectra.

$970 \%$




$9+6$.

$\varepsilon \nabla \mathcal{G}^{\prime} 乙$

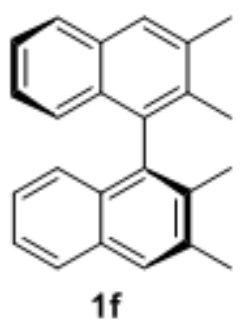

|| $\begin{aligned} & \sum \\ & -\frac{0}{\alpha}\end{aligned}$

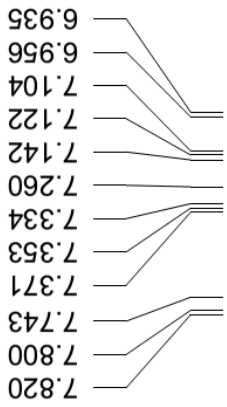

$00 \%$

80.9

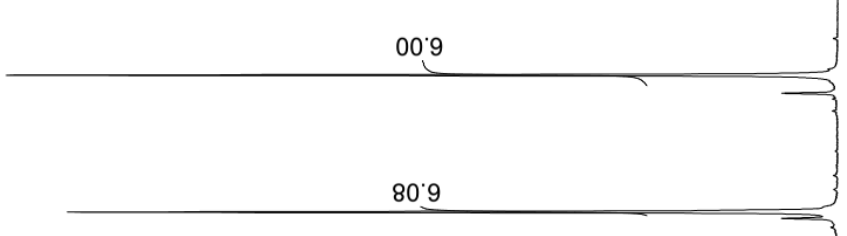

$-N$

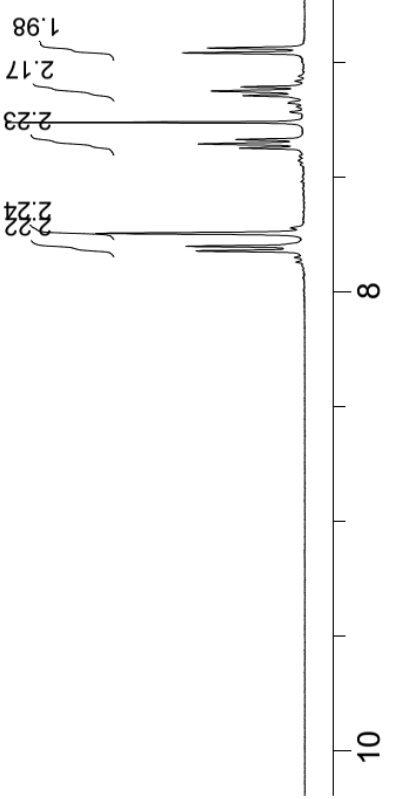


$0<\varepsilon^{\prime} Z$

8เ8' $\mathcal{1}$

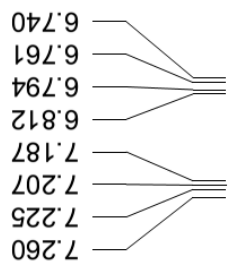

00.9

80.9

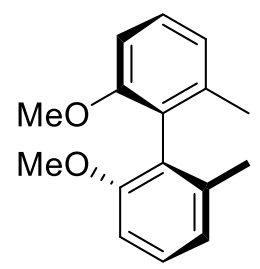

$1 \mathrm{~g}$

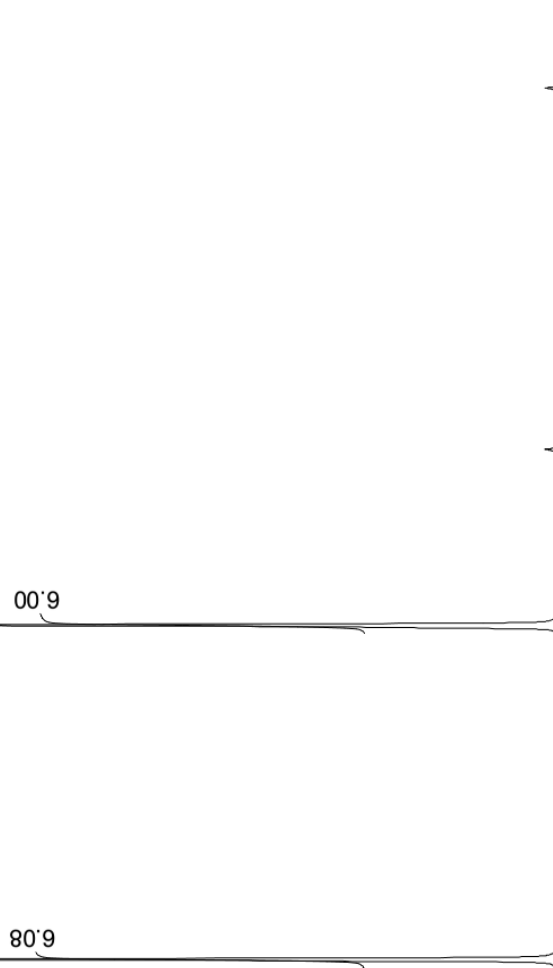

$\sum_{0}$
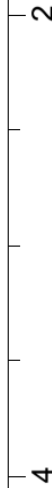

$-6$

sष्ठद.

56.1 


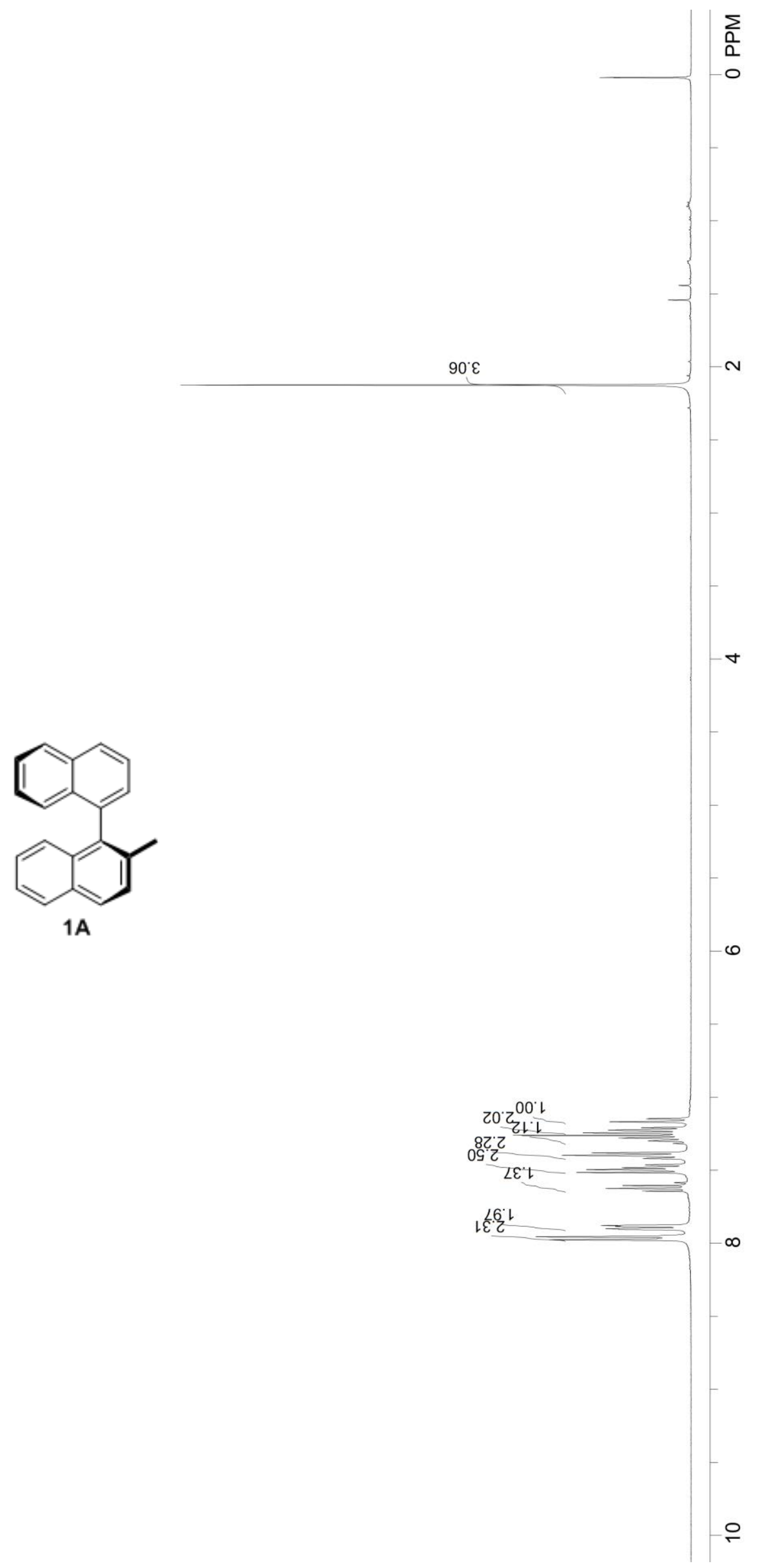

$89 L^{\circ} \mathrm{L}$

9Z2 2

เ9Z'

$\varepsilon 9 \mathrm{Z}^{\circ}$

6LZ'L

เ8E' $L$

$86 \varepsilon^{\circ} L$

$\varepsilon 8 \nabla^{\circ} L$

$96 t^{\circ} \mathrm{L}$

9IG'L

$709^{\circ} \mathrm{L}$

G29. L

$8 \angle 8^{\circ} \mathrm{L}$

$988^{\circ} \mathrm{L}$

$668^{\circ} \mathrm{L}$

$706^{\circ} \mathrm{L}$

$996 . \mathrm{L}$

$9 \angle 6^{\circ} \mathrm{L}$

$1 \mathrm{~A}$ 

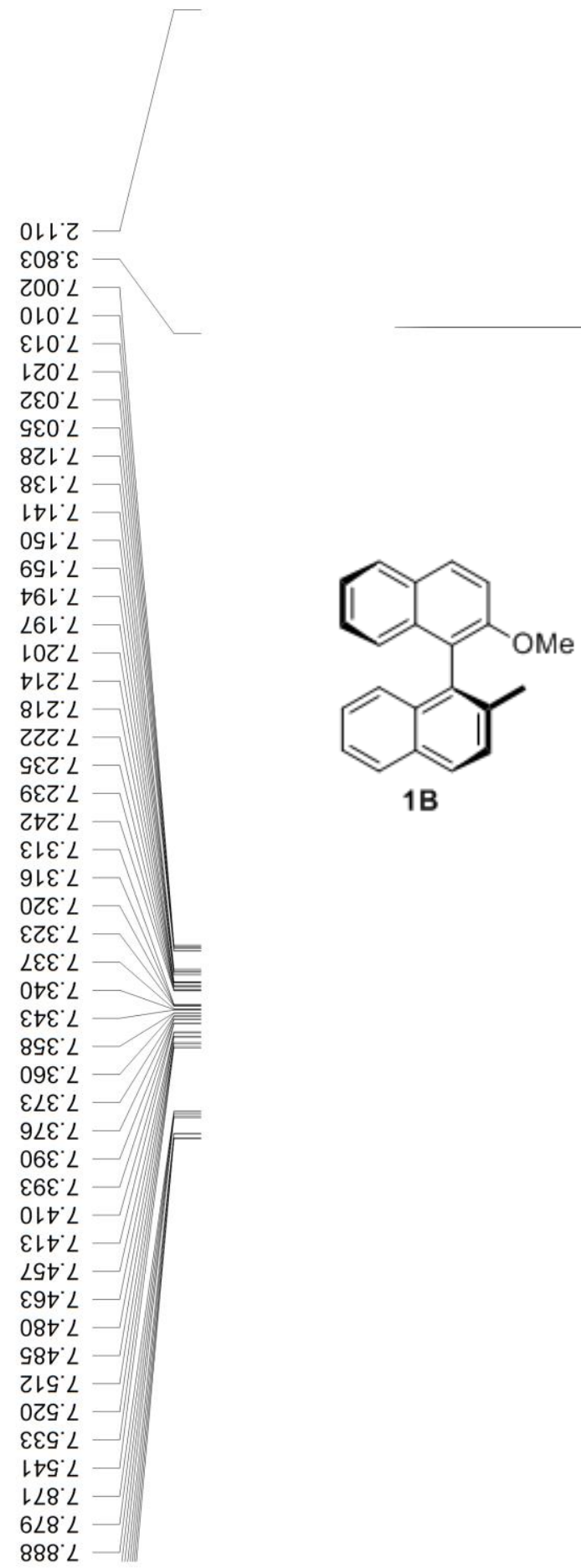

$\angle 6^{\circ} \mathrm{Z}$ 
8LLL

$980^{\circ} 2$

$8 E L L$

$89 L \mathrm{~L}$

$60 Z$ L

ILZ L

GZZ L

$8 Z 2$ L

$0 t Z \cdot L$

GSZ' $L$

ZLZ L

GLZ L

$\varepsilon 6 Z^{\circ} L$

G9. $L$

G8E' $L$

$\angle 6 \varepsilon^{\circ} \angle$

6Lt L

SEt $L$

SSt $L$

Z8t $L$

Z98 $\mathrm{L}$

$\varepsilon \angle 8^{\circ} L$

ZE6 $\angle$

$296^{\circ} \mathrm{L}$

$086^{\circ} \mathrm{L}$

$200{ }^{\circ} 8$

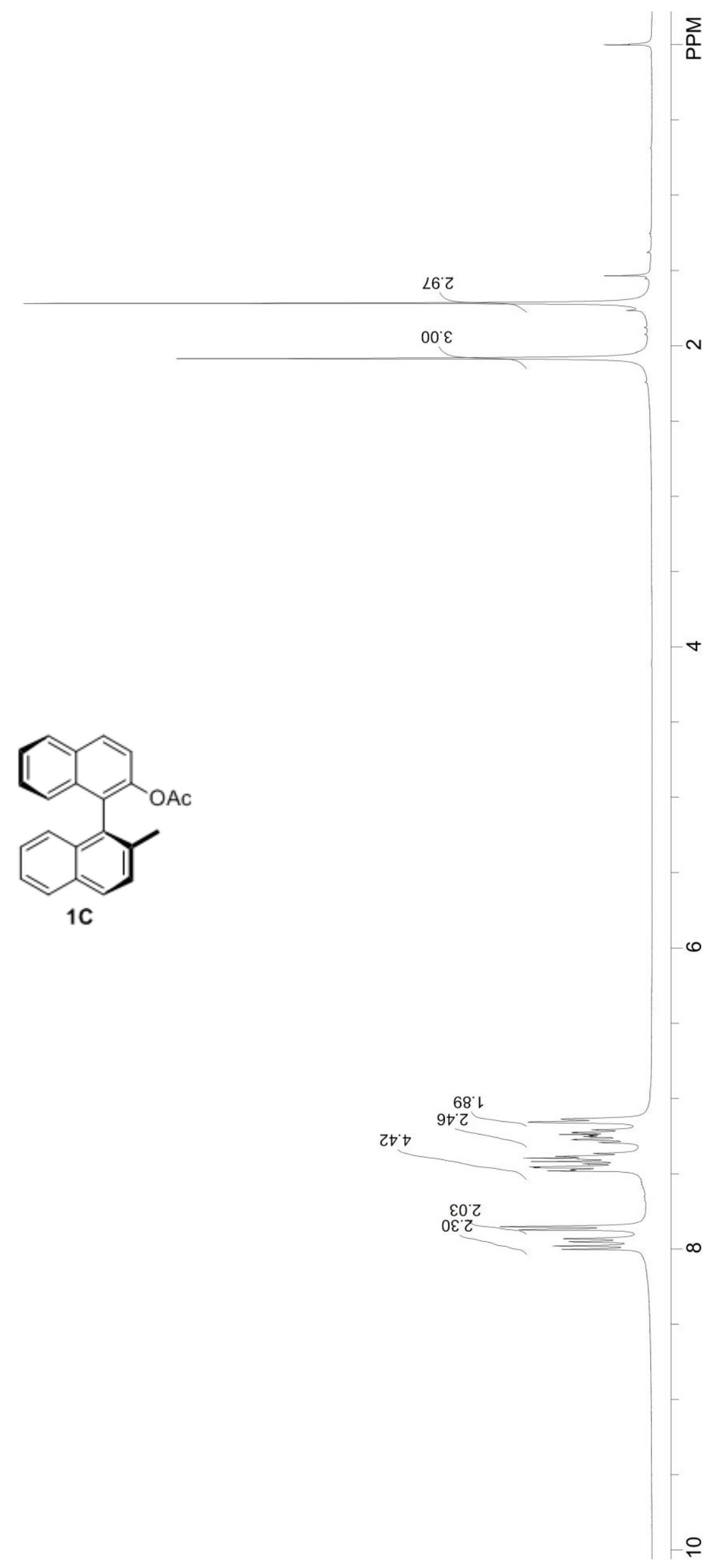




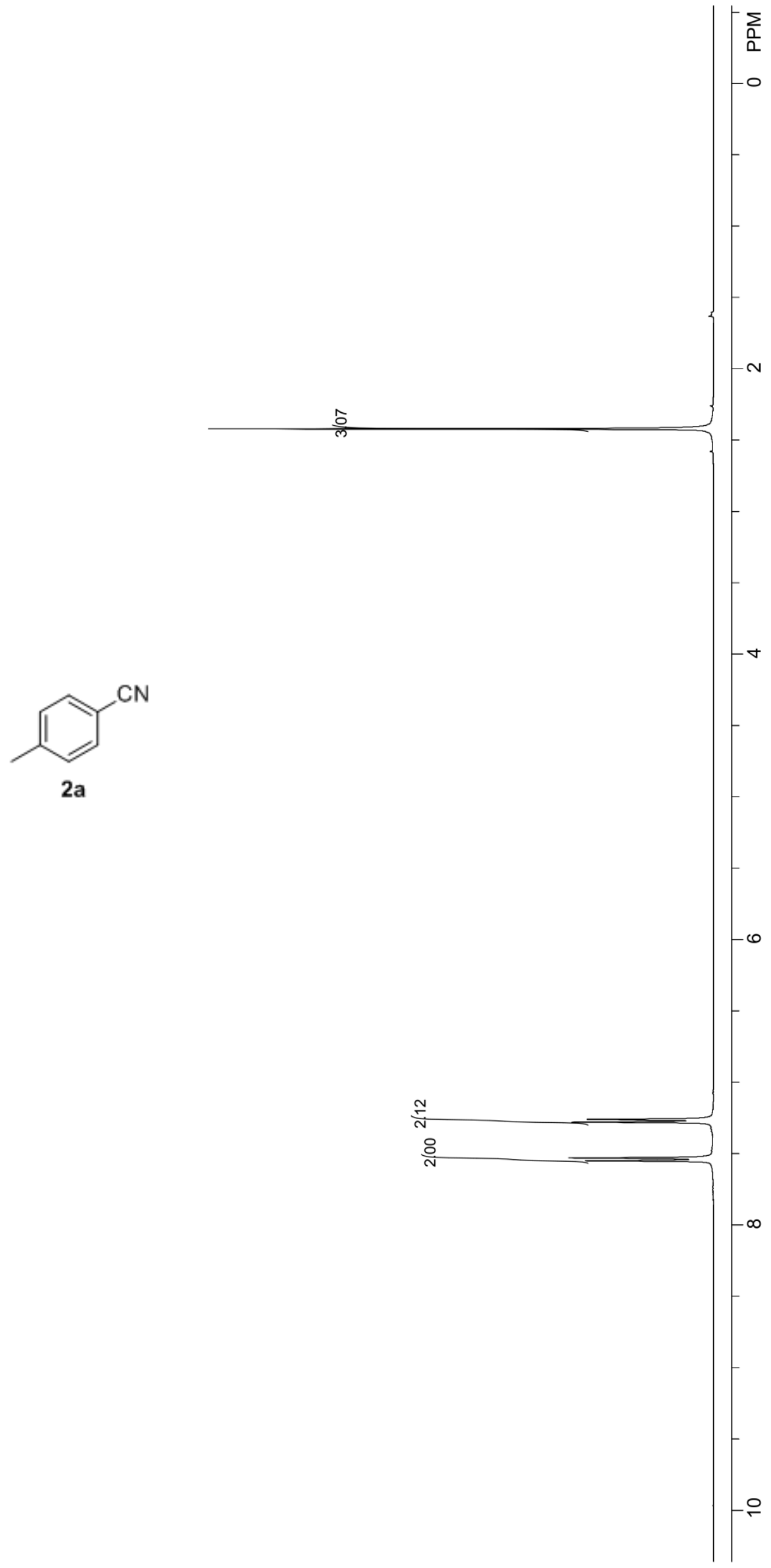

$09 Z^{\circ} \angle$

$08 Z^{\circ} \mathrm{L}$

IES'L

ISG' $L$ 


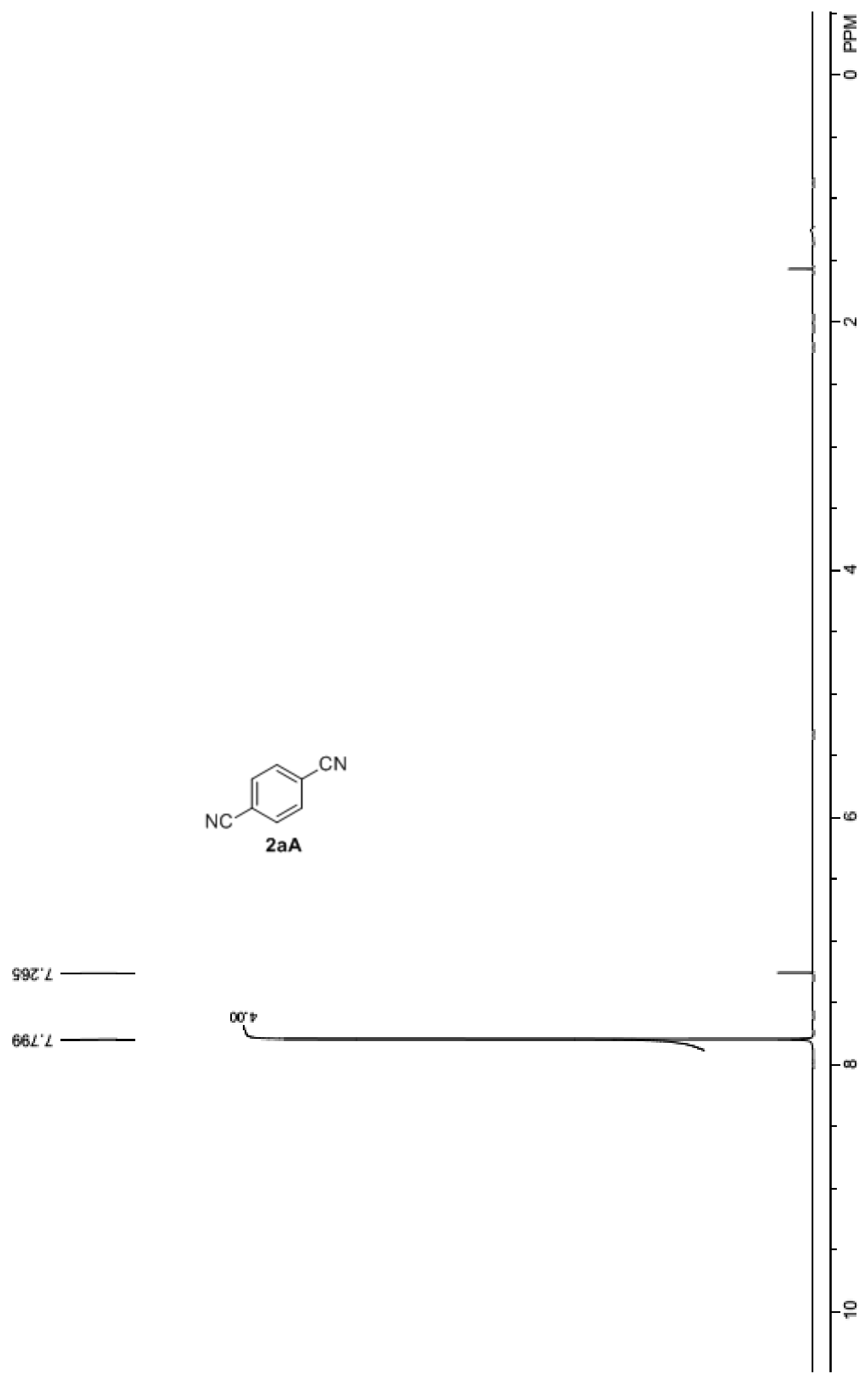




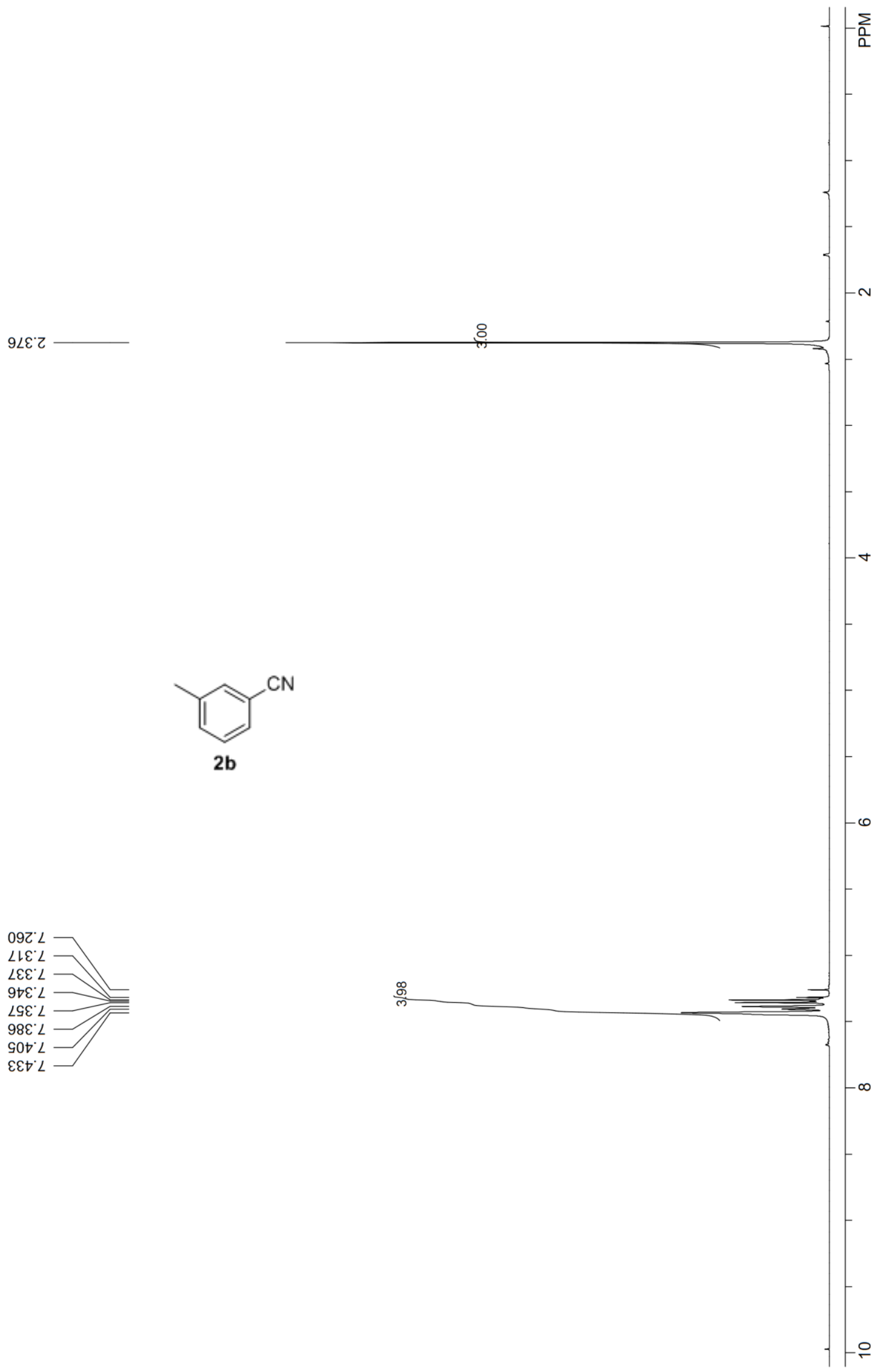




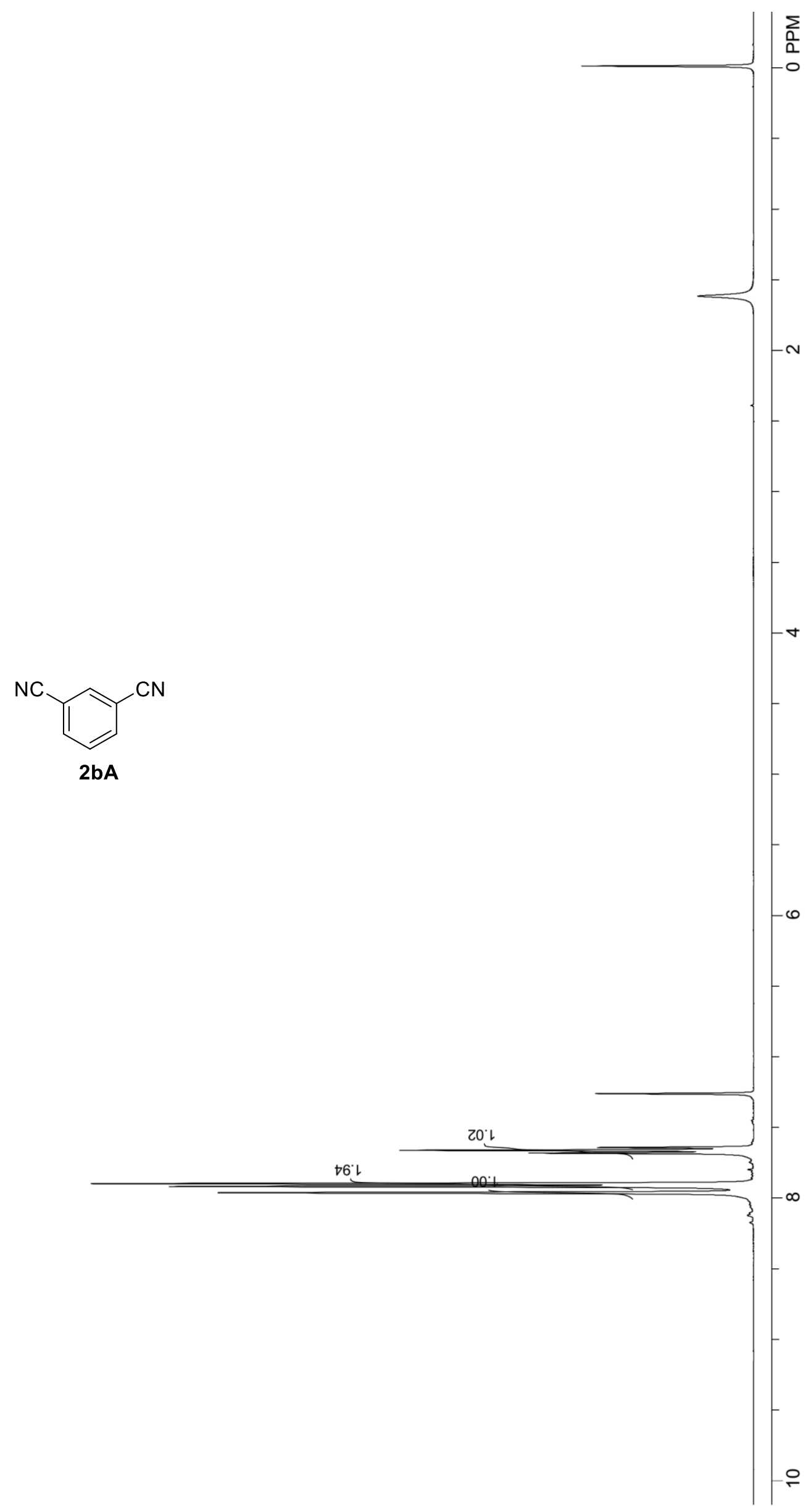

097.2

$\mathrm{\nabla} 9^{\circ} \mathrm{L}$

$199^{\circ} \mathrm{L}$

$189^{\circ} \mathrm{L}$

$868^{\circ} \mathrm{L}$

816.2

$296^{\circ} \mathrm{L}$ 


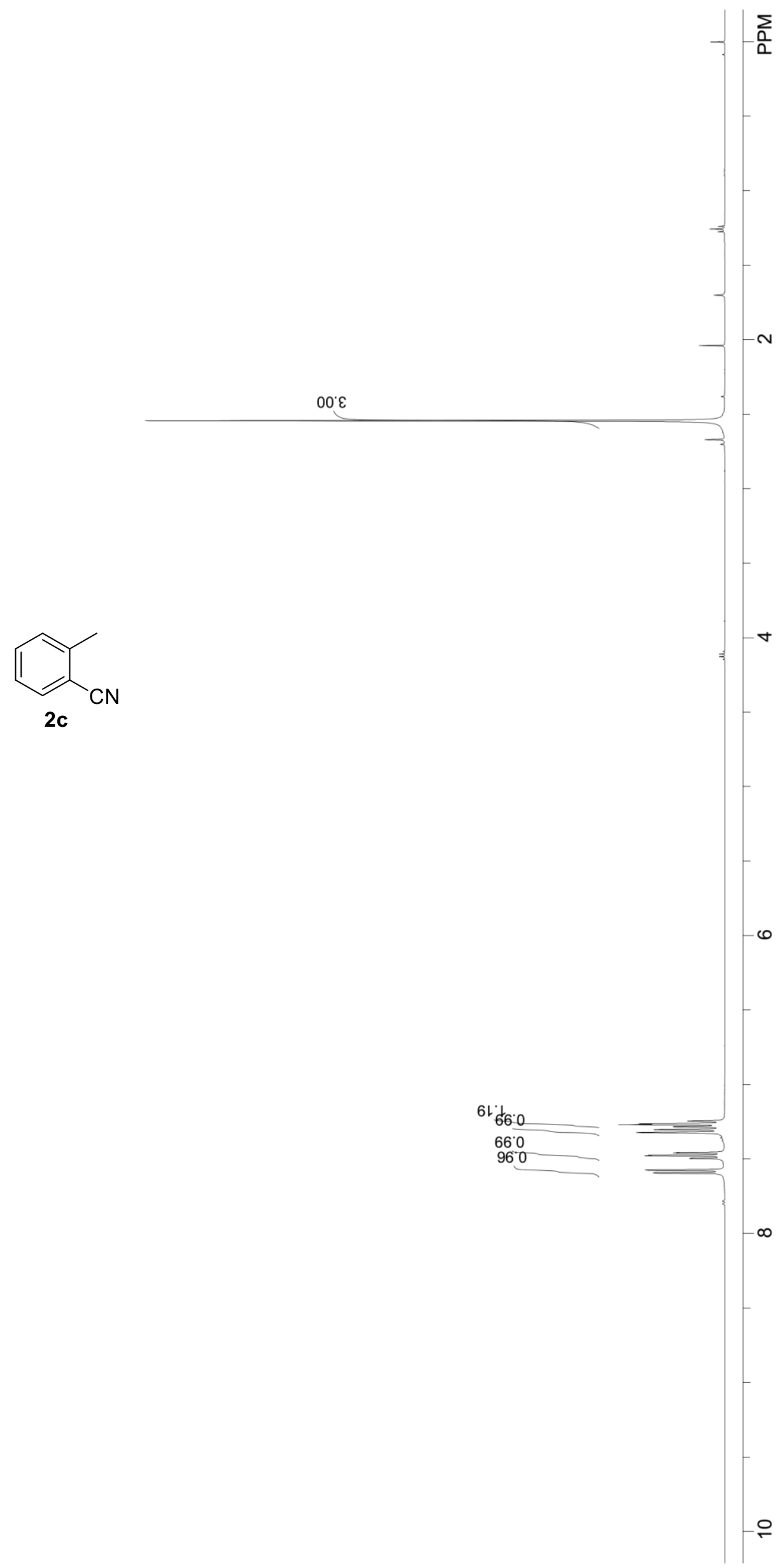

9tZ

G9Z L

$69 Z^{\prime} L$

$\neg 8 Z^{\circ} L$

$\varepsilon 0 \varepsilon^{\circ} L$

¿ZE' $L$

69t.

$8 \angle t^{\circ} L$

$867^{\circ}$

$\nabla \angle S^{\circ} L$

E6G'L 


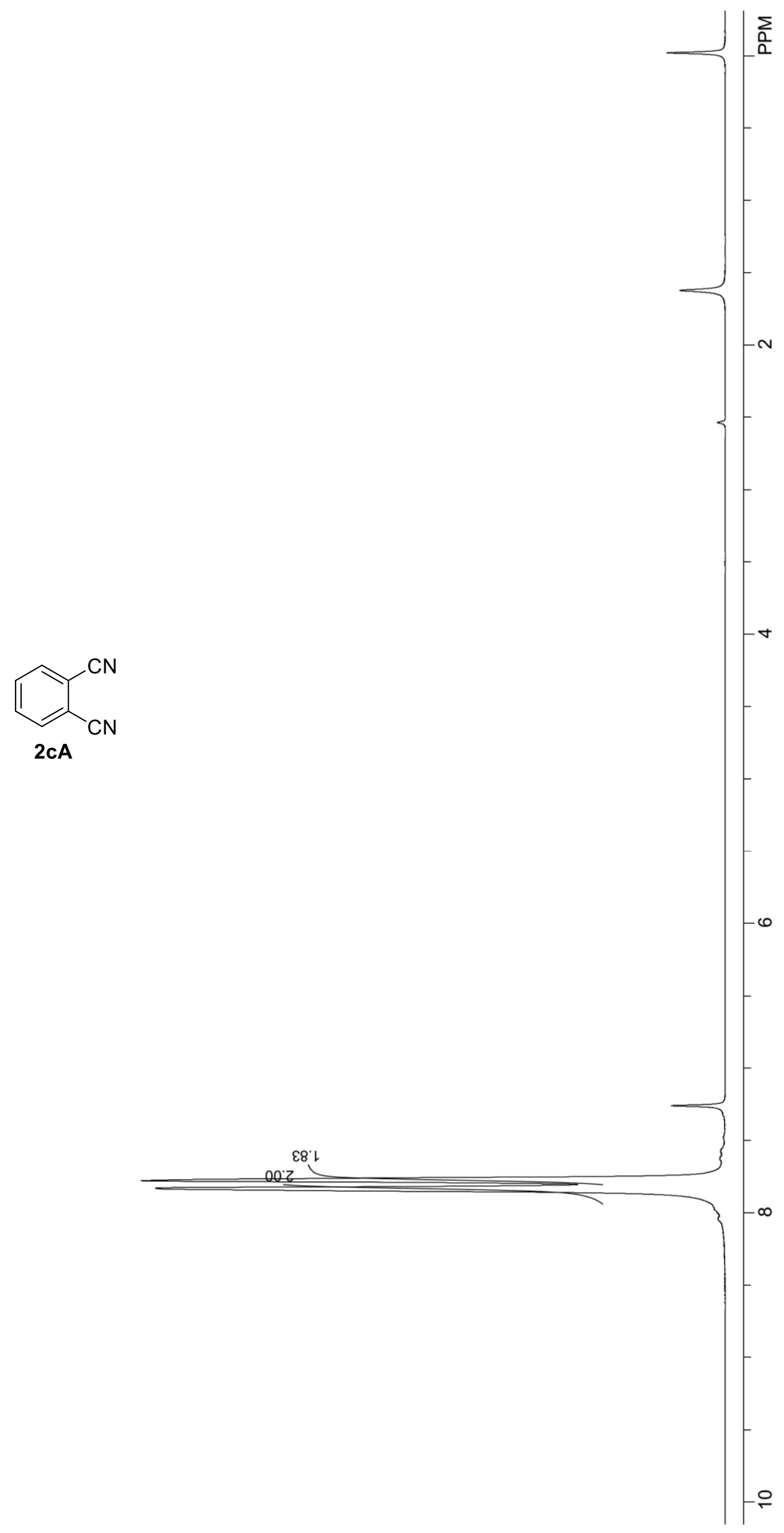




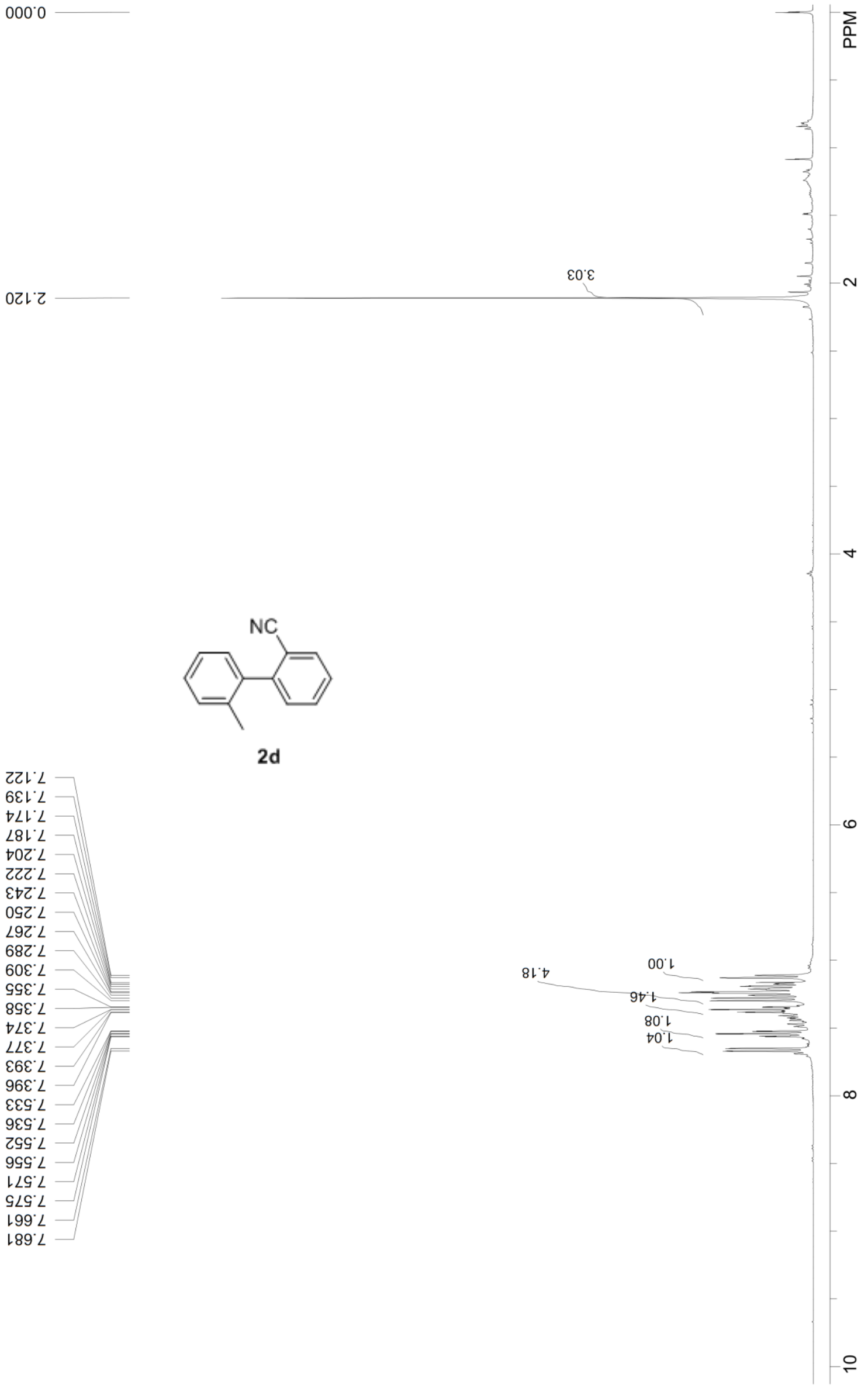




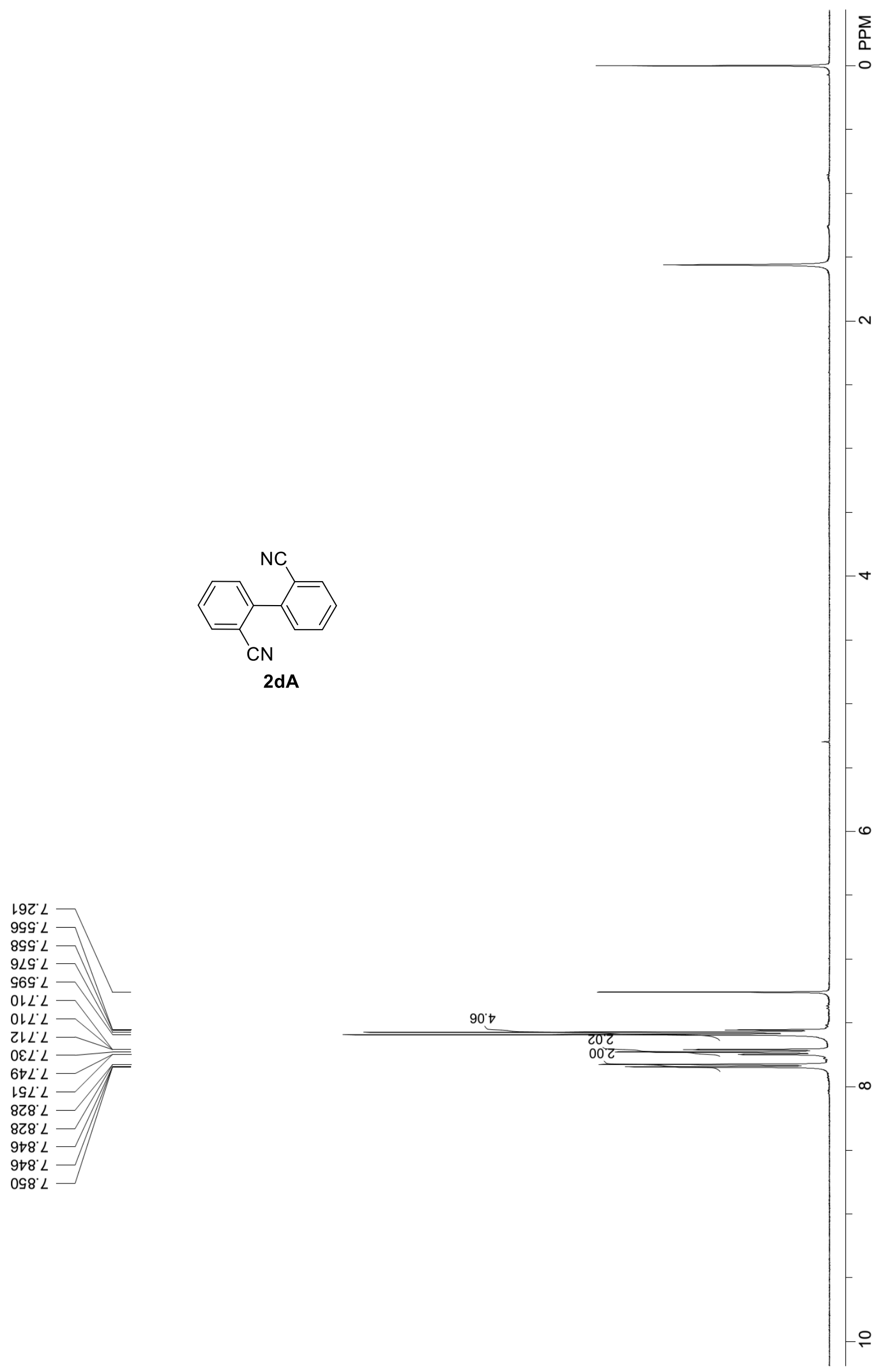



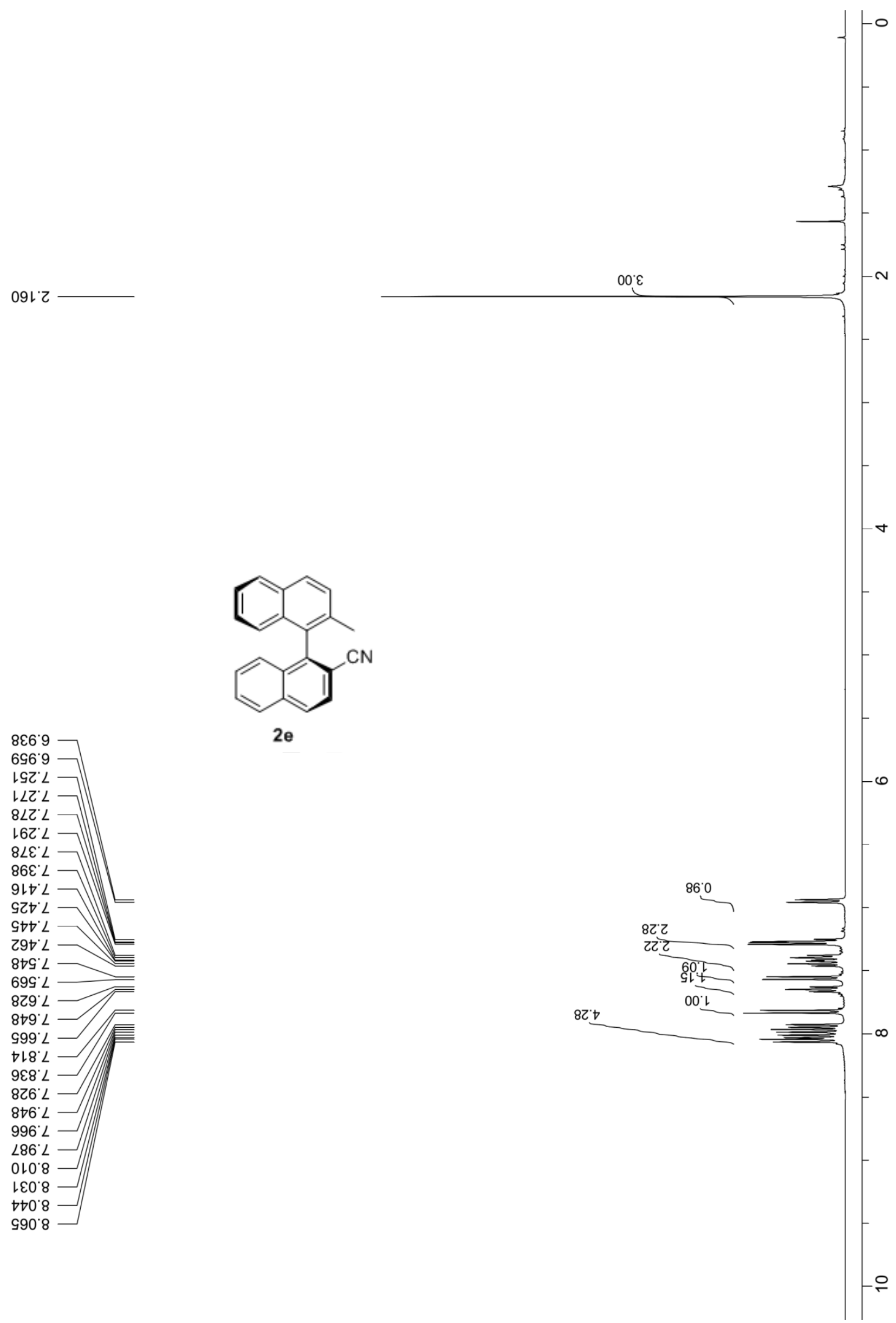

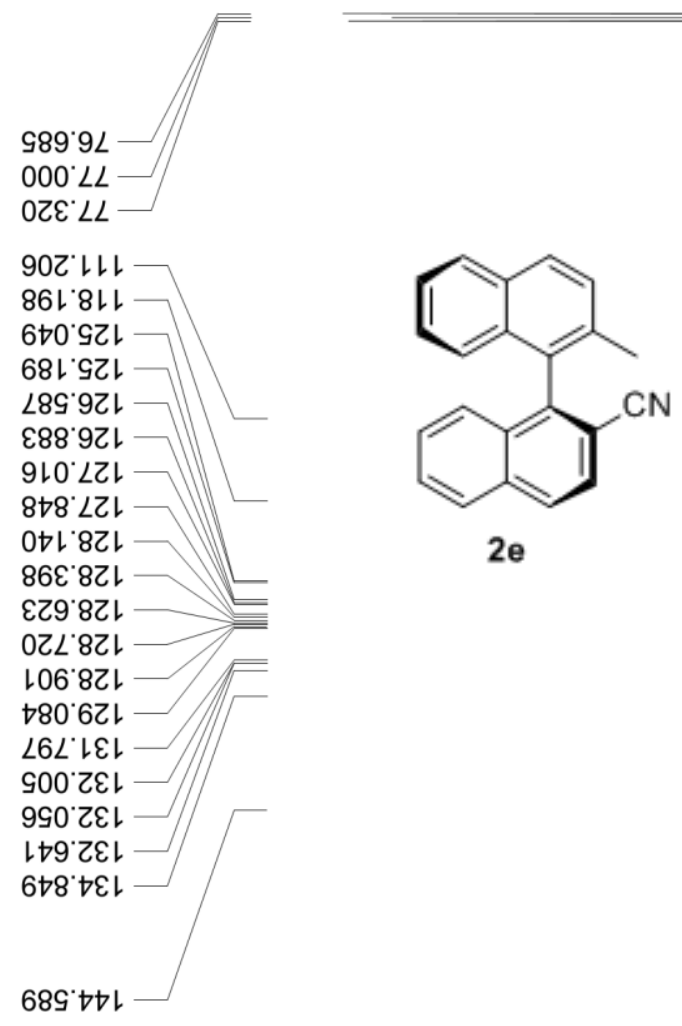


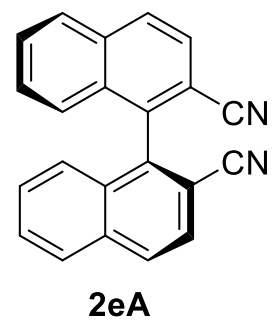

89.'

192.

ZZt L

Ztた L

E9t.

$979^{\circ} \angle$

$999^{\circ}$

$789^{\circ} \angle$

$6 \varepsilon 8^{\circ} \mathrm{L}$

$098^{\circ} \mathrm{L}$

$\mathrm{ZZO} 8$

$\triangleright 70.8$

EZL. 8

เทเ:8 -

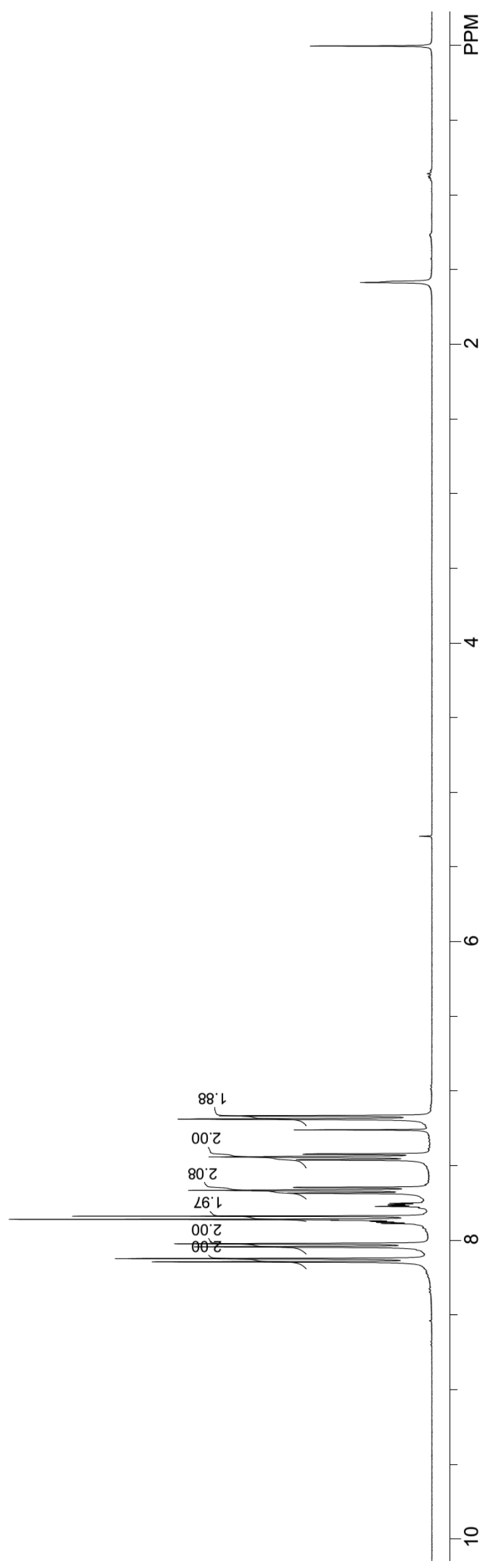


$00 Z 乙$

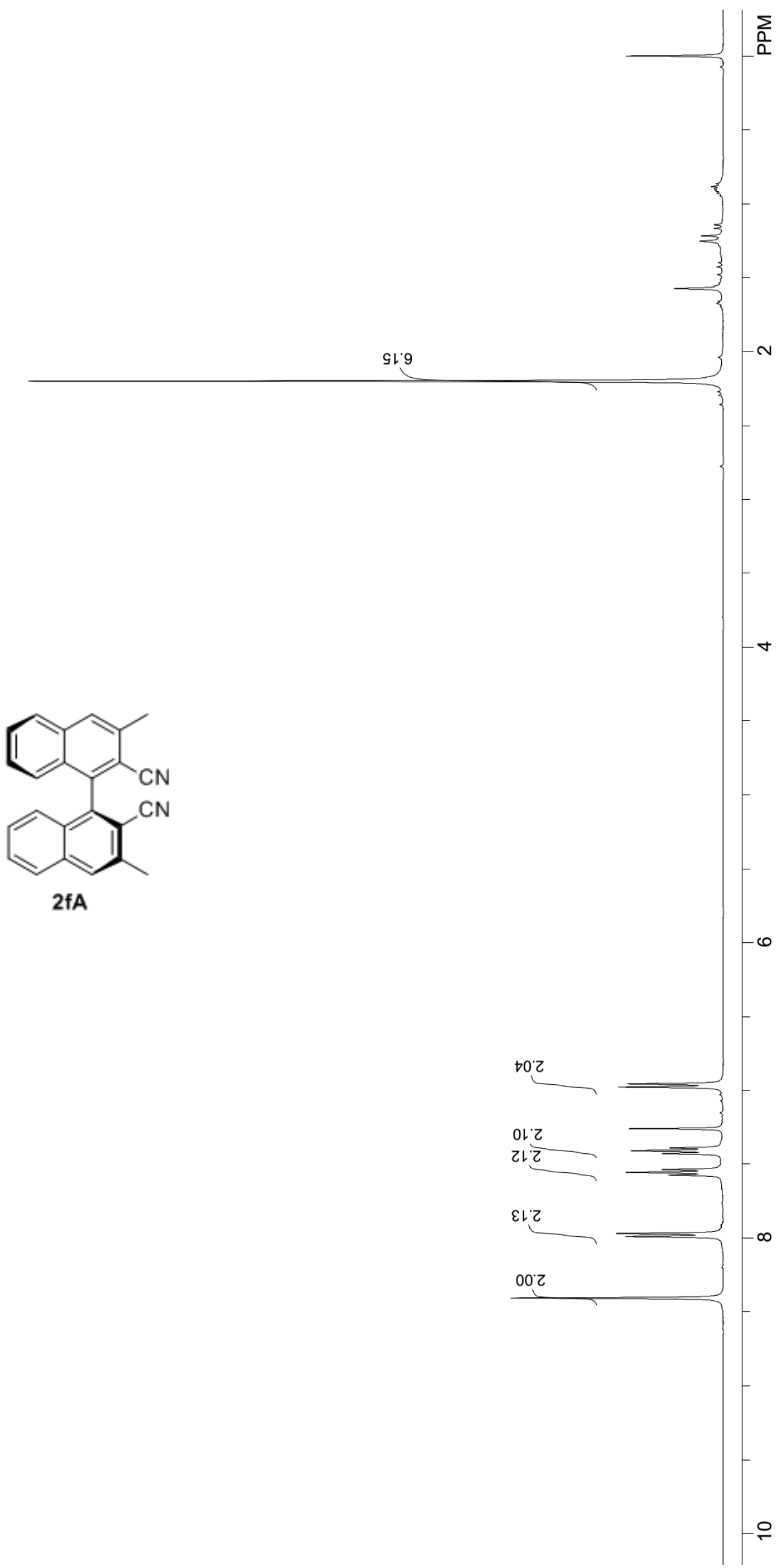



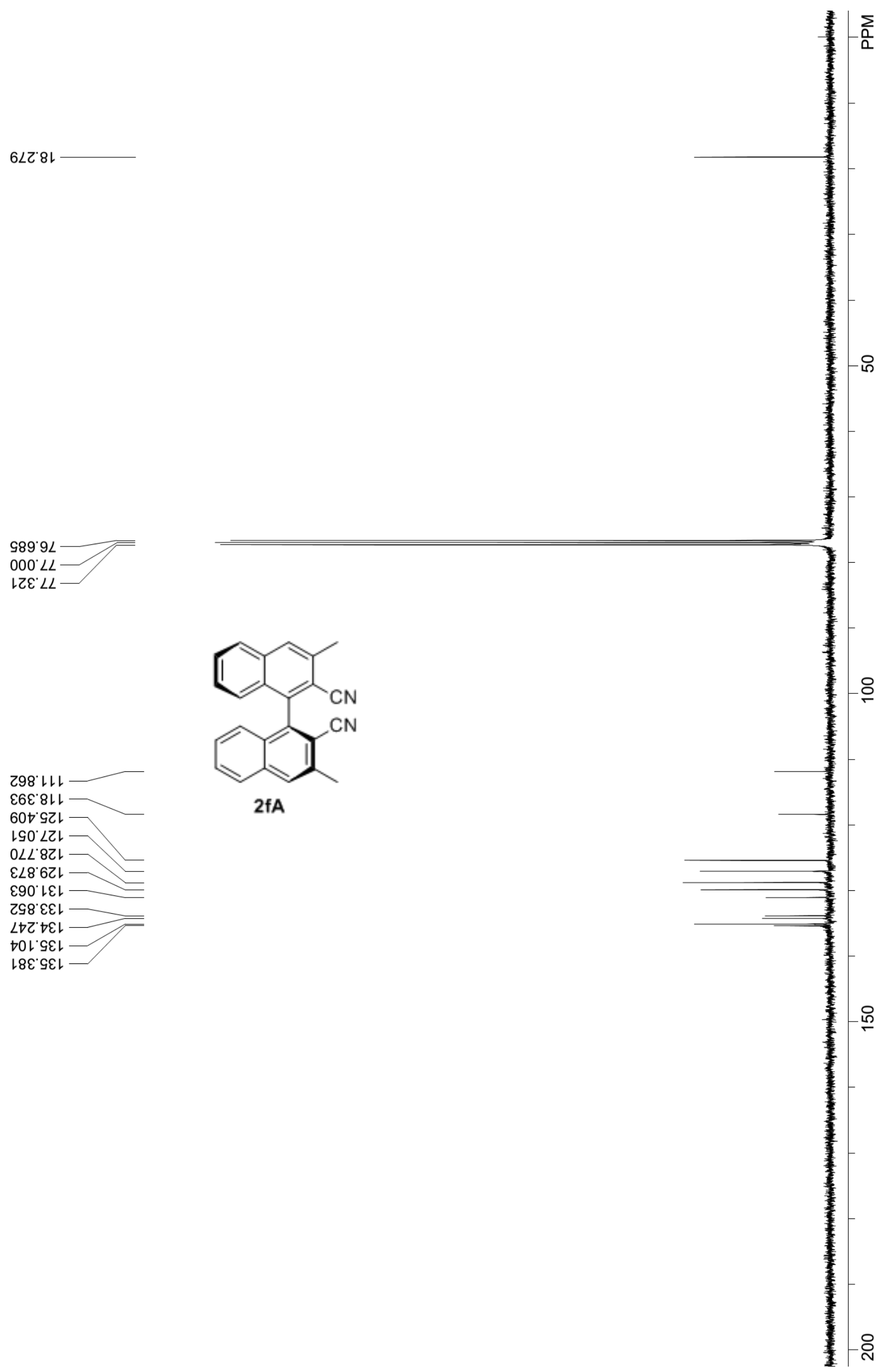
$108^{\circ} \varepsilon$

$601 \%$

GILL

6ILL

GZI.

$8 \mathrm{~L} \cdot \mathrm{L}$

$602 \angle$

ZIZ

8ट乙

เEZ

$\downarrow \varepsilon Z$ L

09Z L

$\angle \varepsilon \varepsilon$ ' $\angle$

$8 \nabla \varepsilon$.

$\angle G \varepsilon^{\circ} \angle$

$09 \varepsilon^{\circ} L$

$6 \angle \varepsilon^{\circ}$

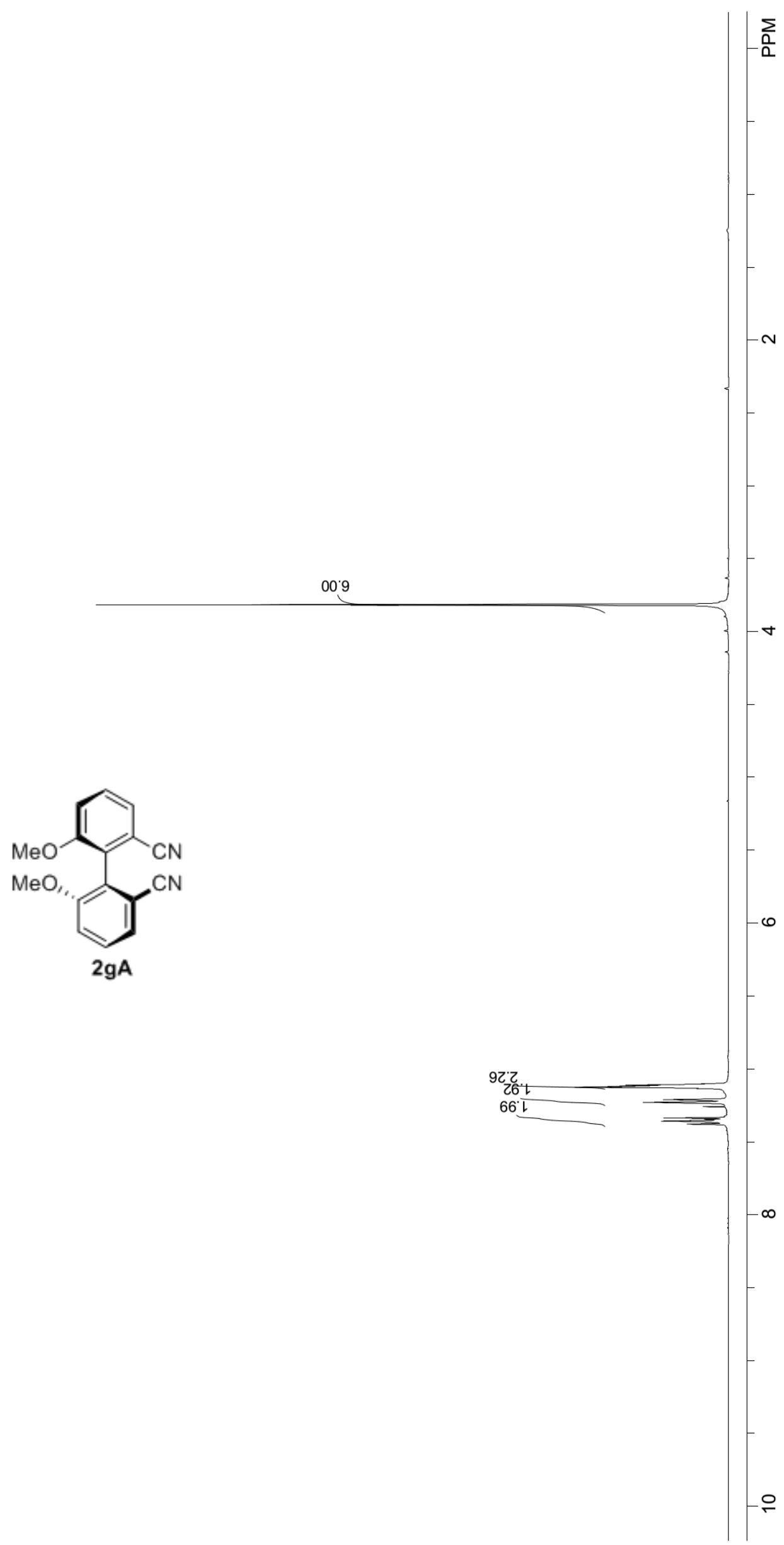




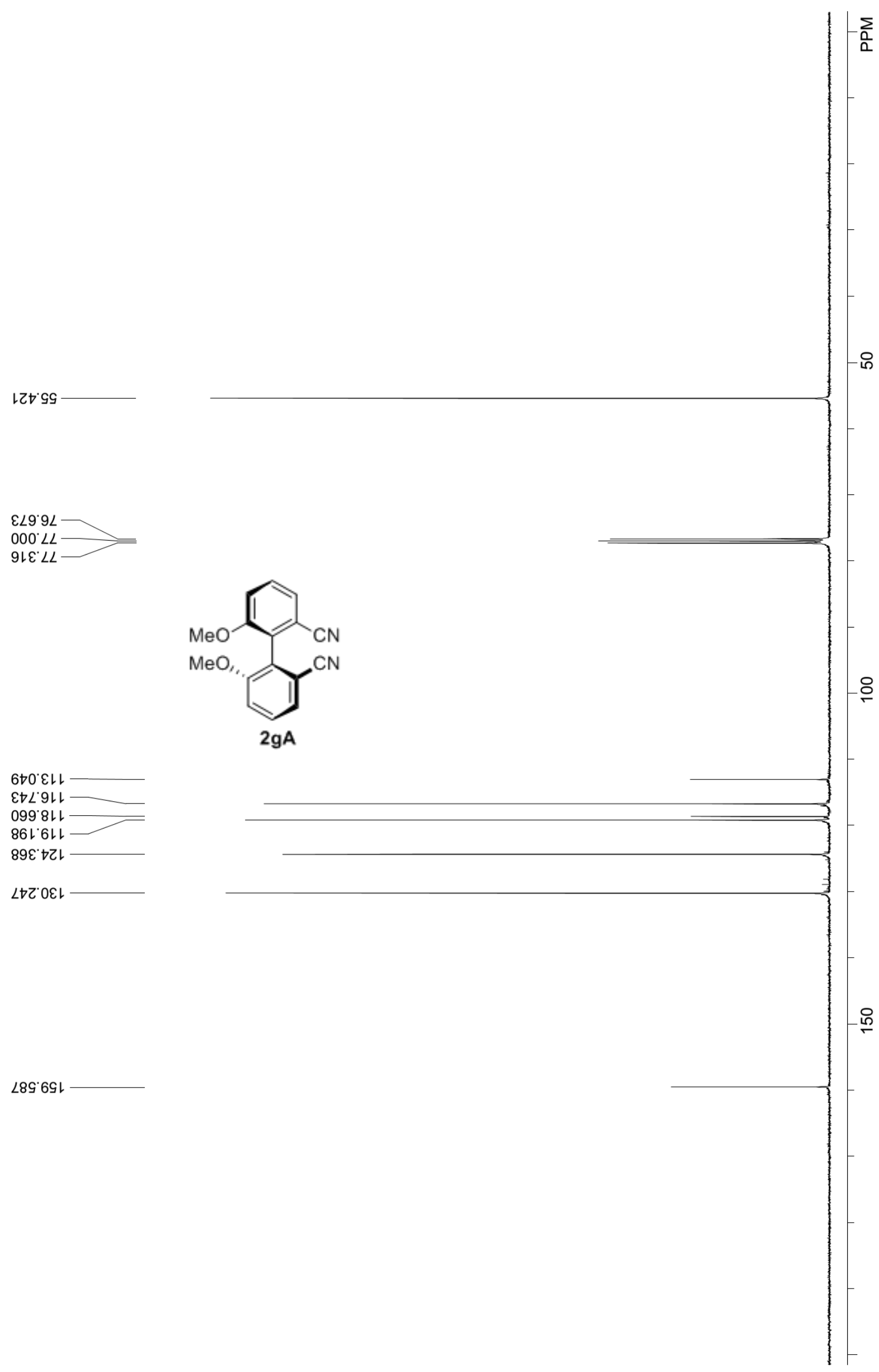




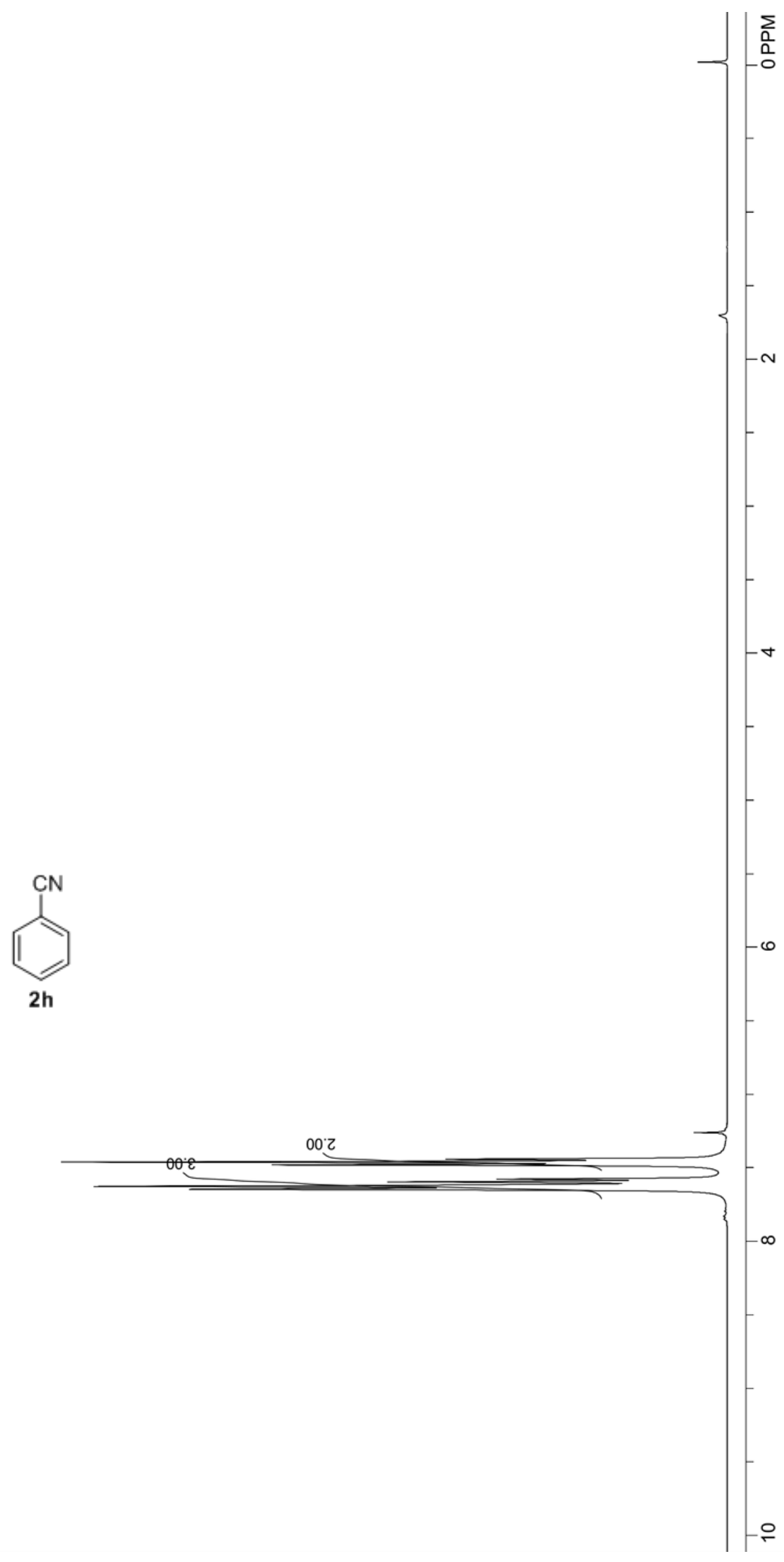

$192 \mathrm{~L}$

1972

$08 \mathrm{t}^{\circ} \mathrm{L}$

$\angle L G^{\circ} L$

969' 2

GZ9.

$829^{\circ} \mathrm{L}$

$9+9^{\circ} \mathrm{L}$ 
५५८ $\varepsilon$

$\dagger \varepsilon 6^{\circ} 9 \square$
$996.9 \square$

$09 Z^{\prime} L$

$G \angle S^{\circ} L$

Z69' $L$

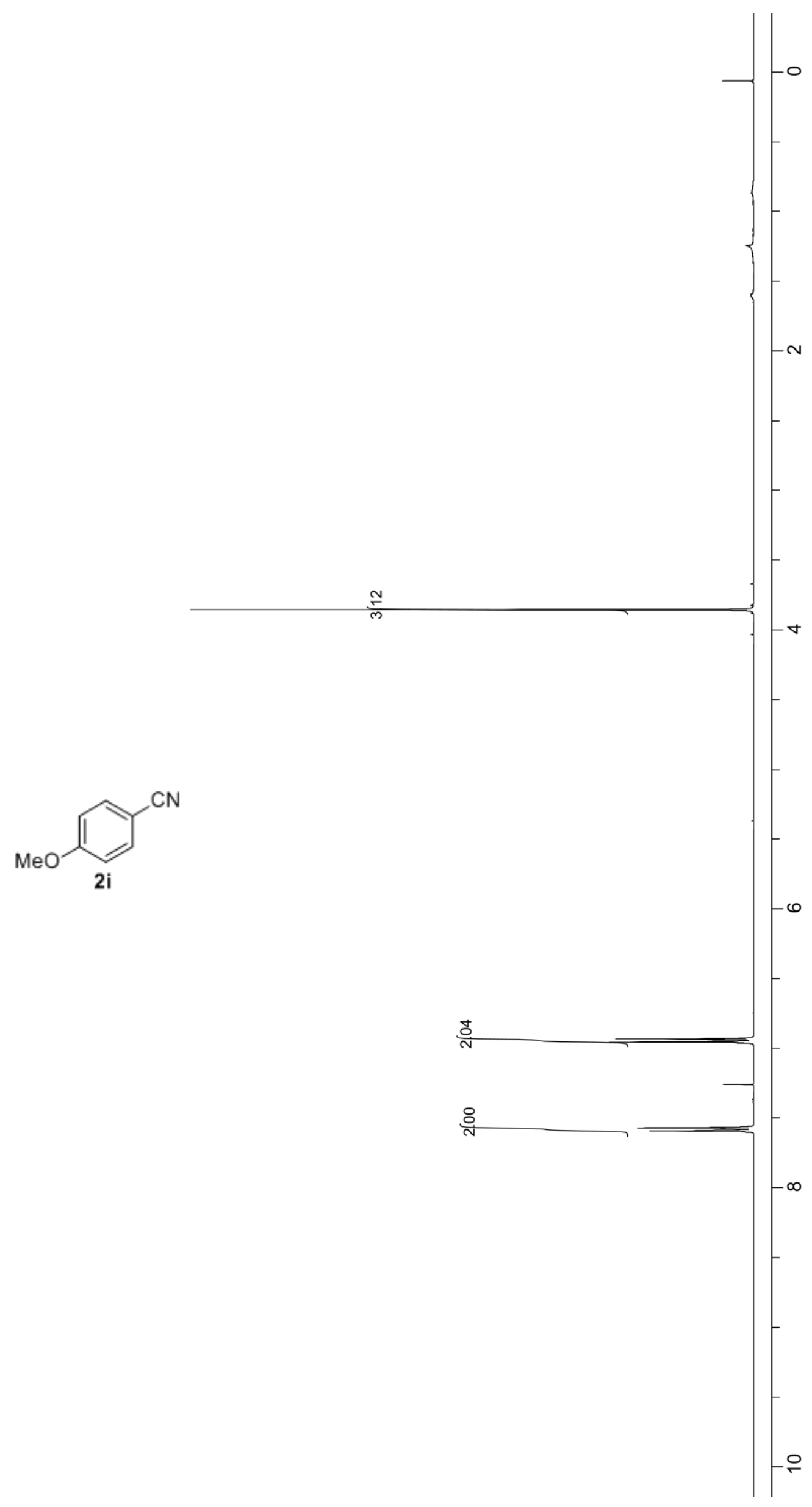




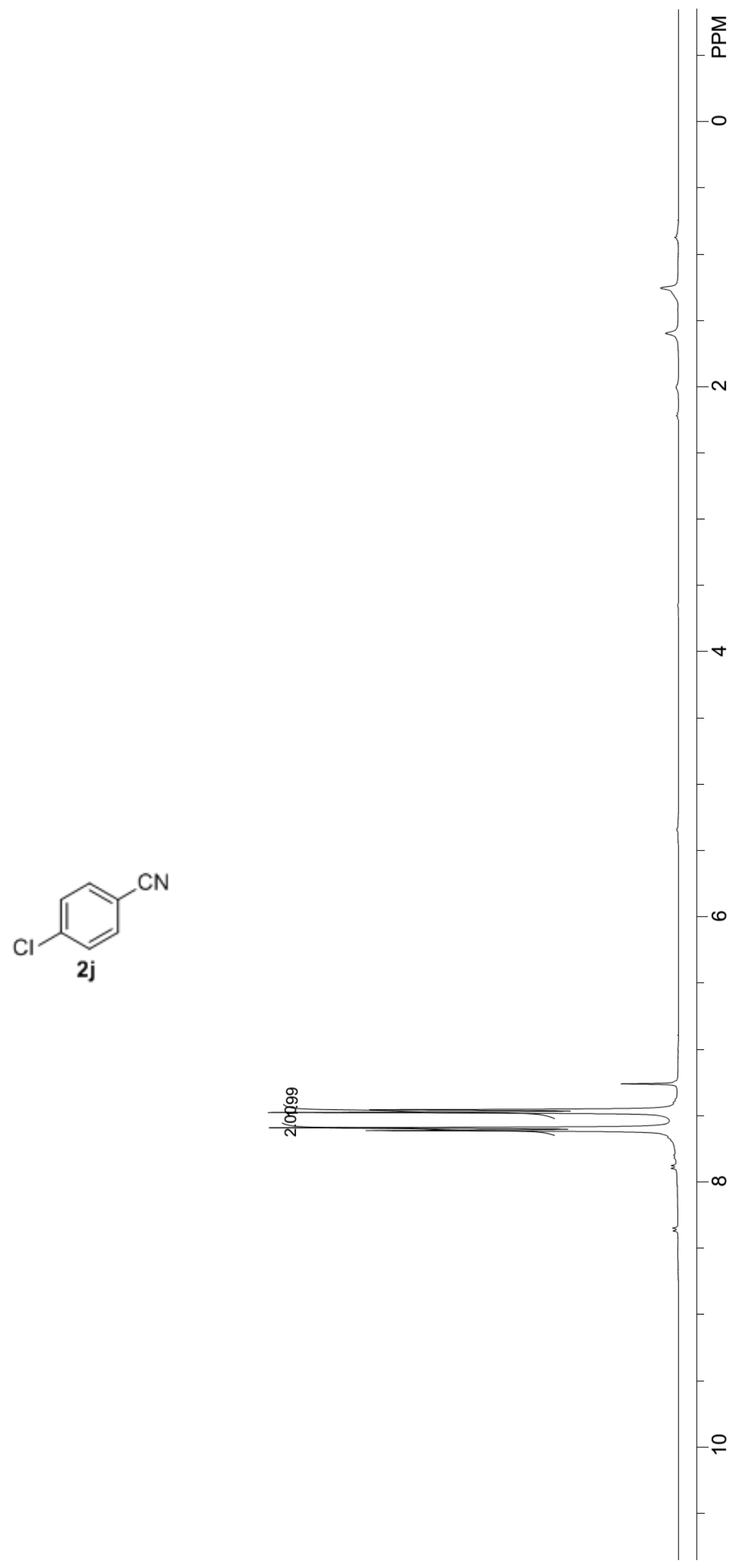

092 L

9St $L$

Z6G' $L$

EI9. $L$ 


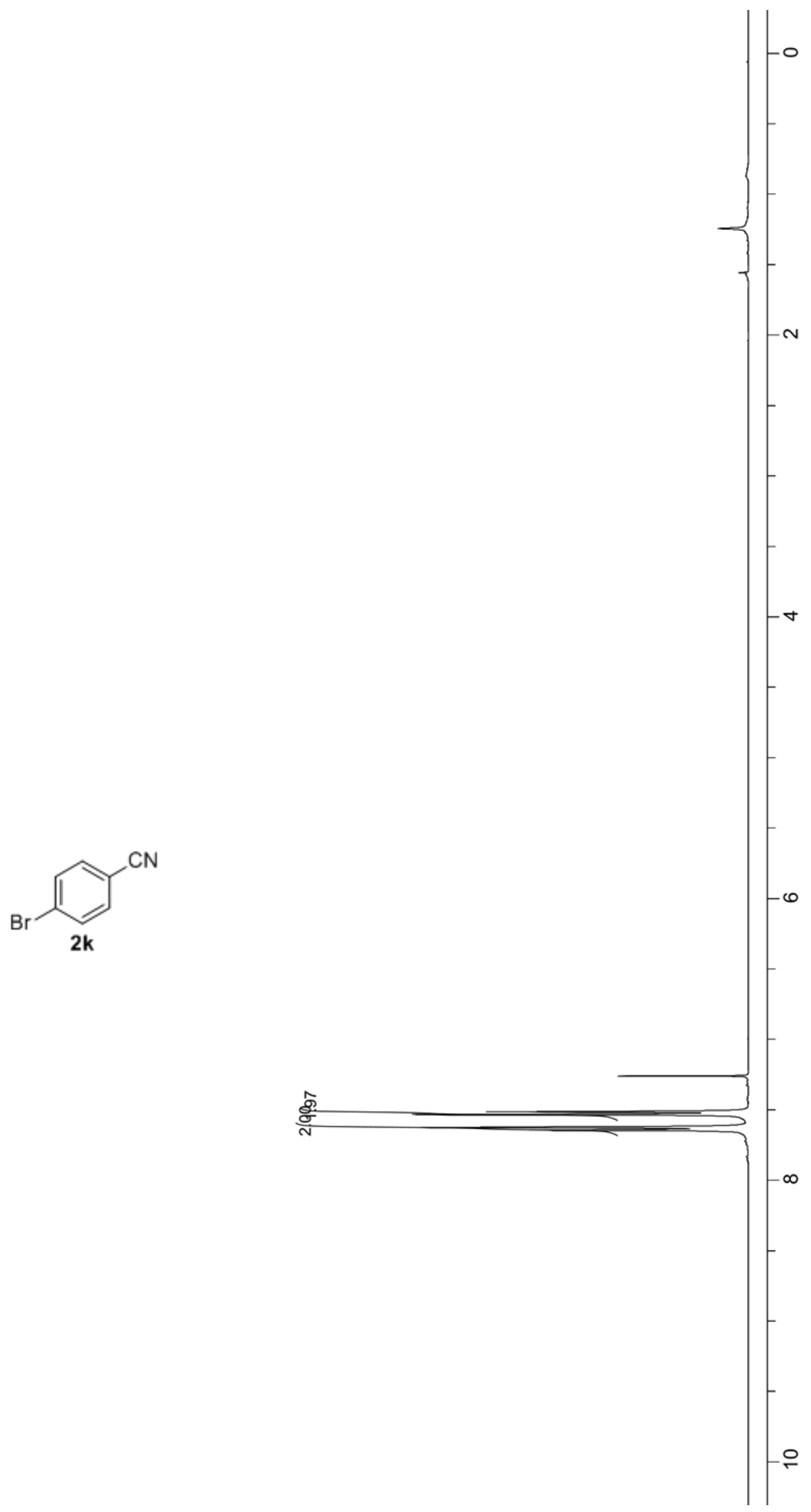

09Z' L

GIG'L

EEG $L$

9ES $L$

LZ9' $L$

$\angle Z 9^{\circ} \mathrm{L}$

¿E9. $\mathrm{L}$

$\varepsilon \downarrow 9^{\circ} L$

$\angle t 9^{\circ} \mathrm{L}$ 


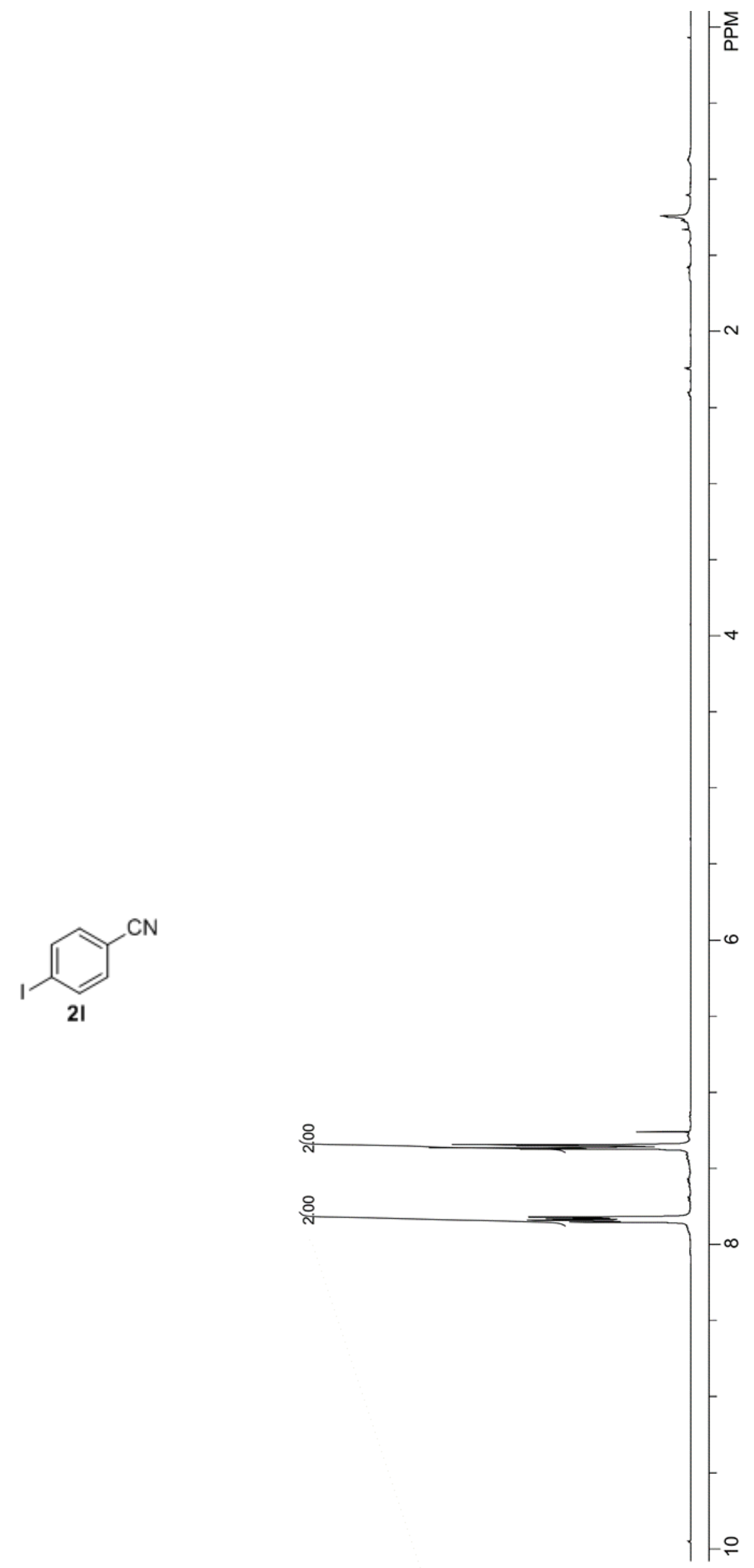


$129^{\circ} Z$

$-$

$09 Z L$

$0 t L L=$

0ع0.8

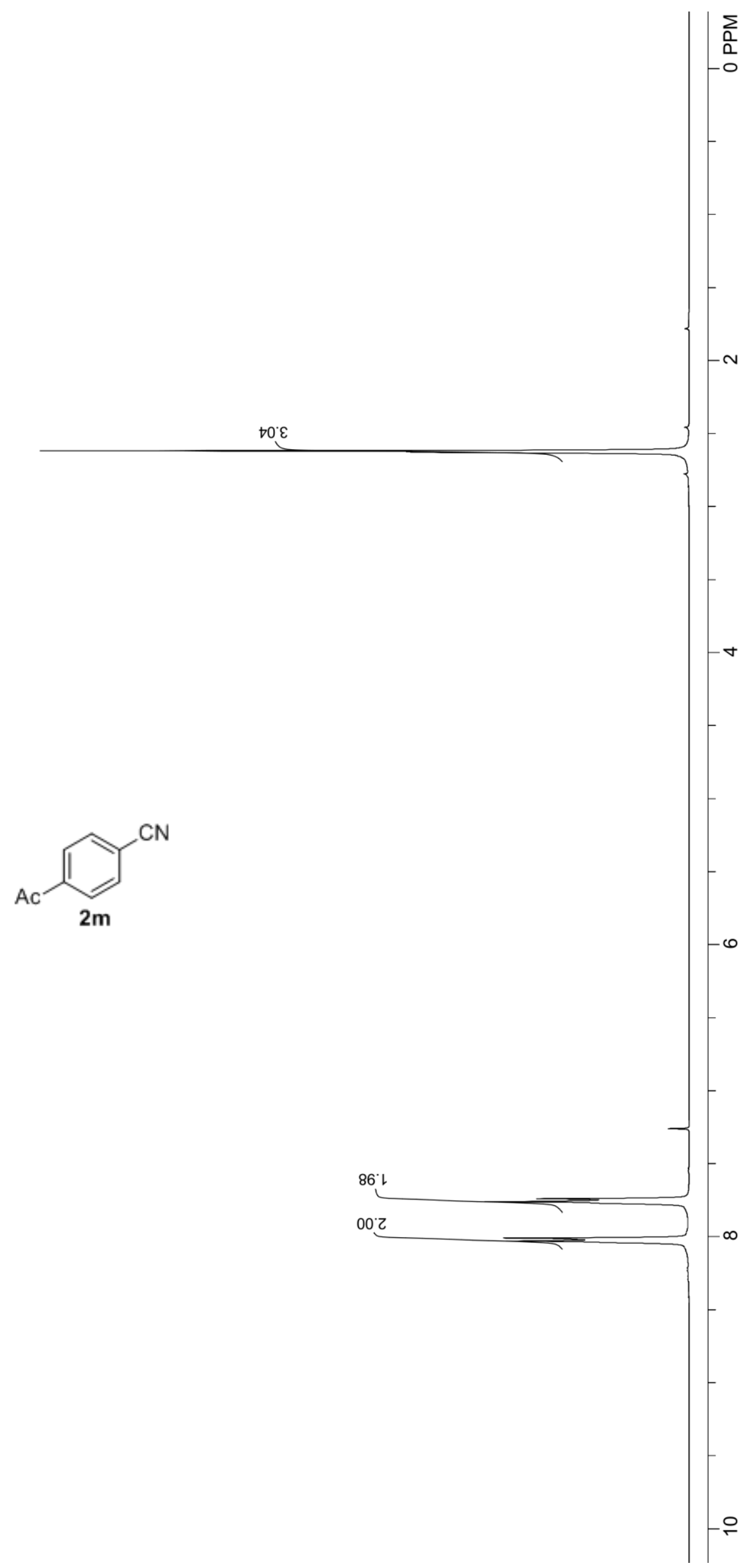




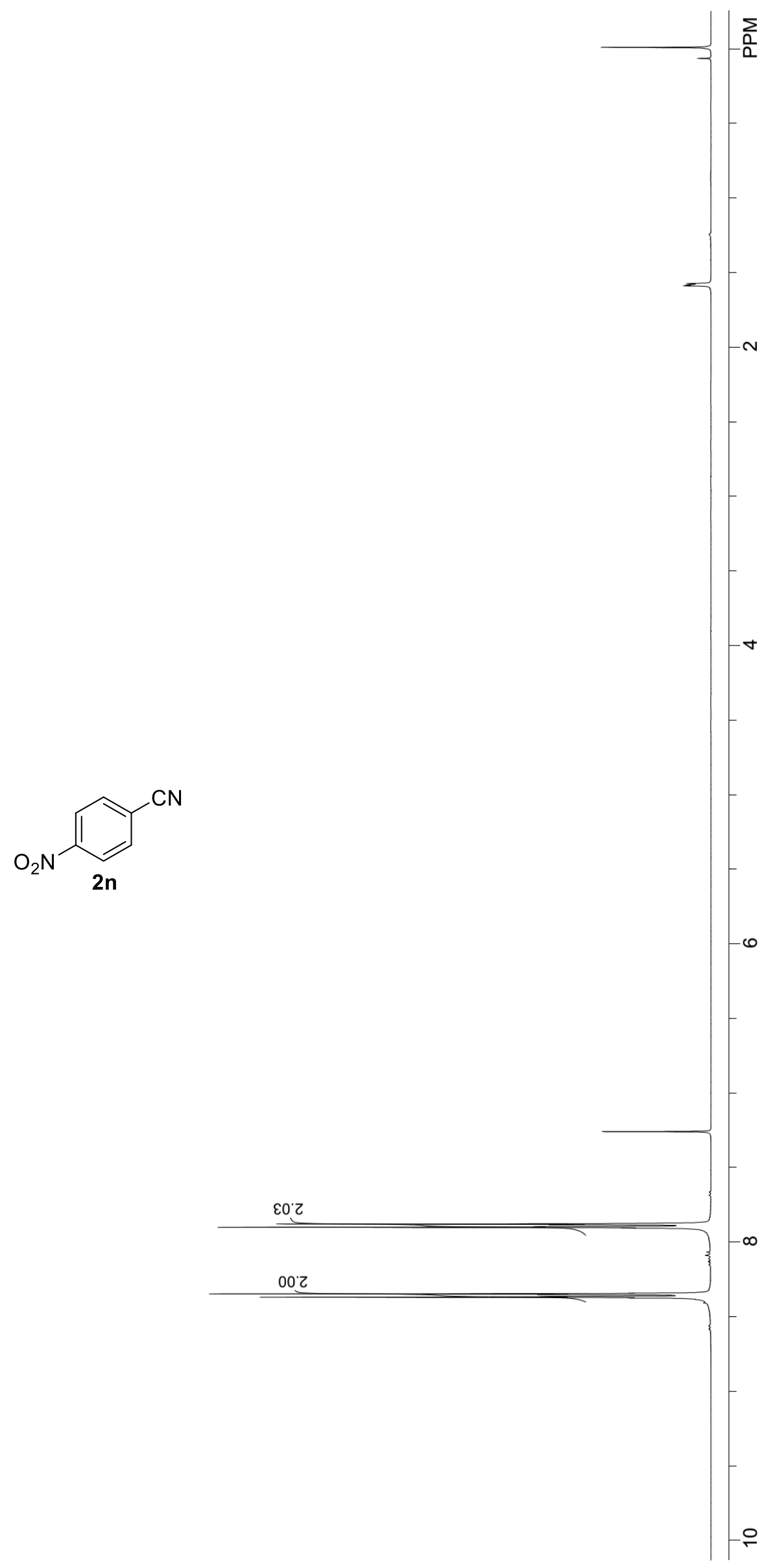




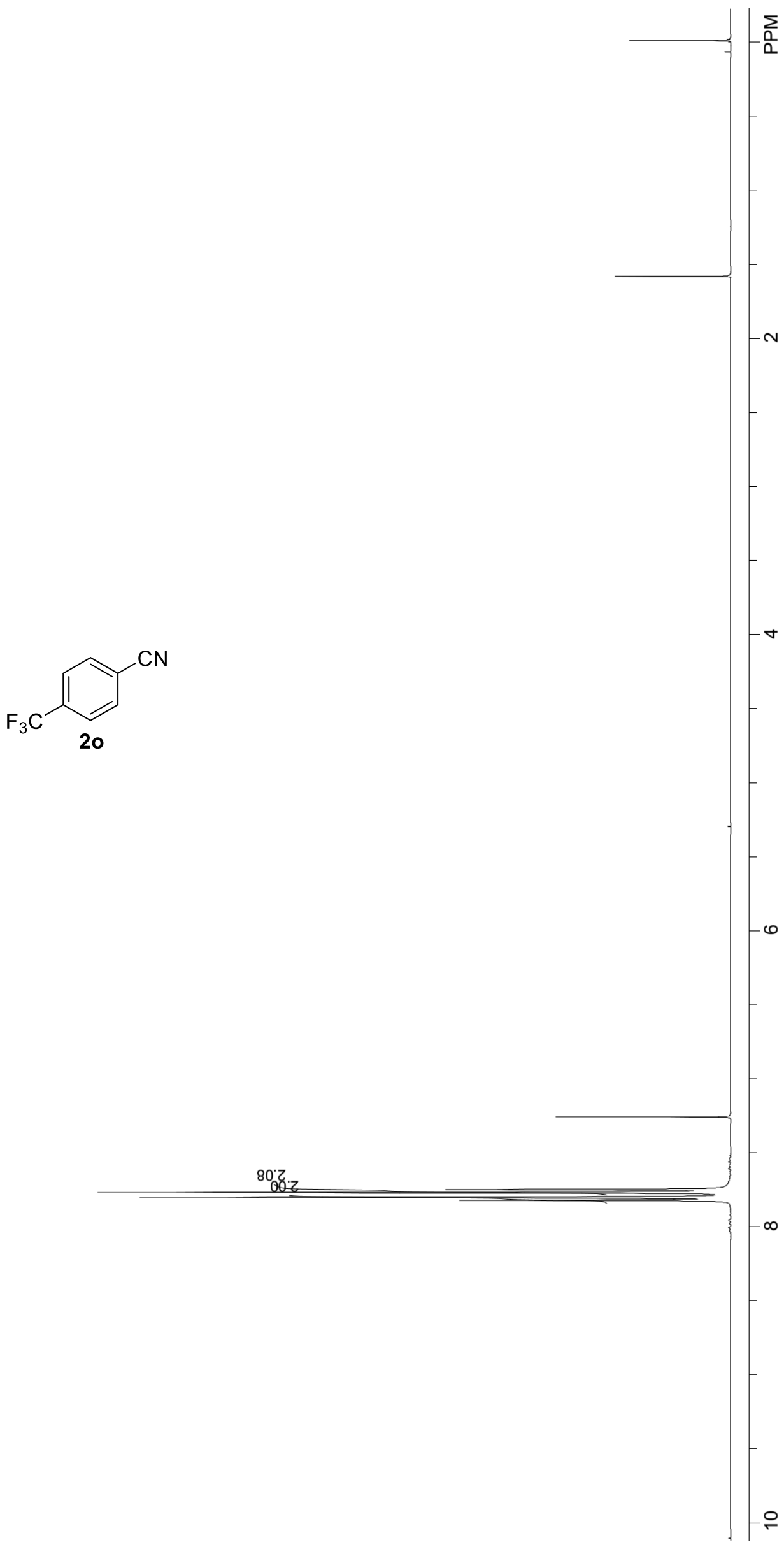



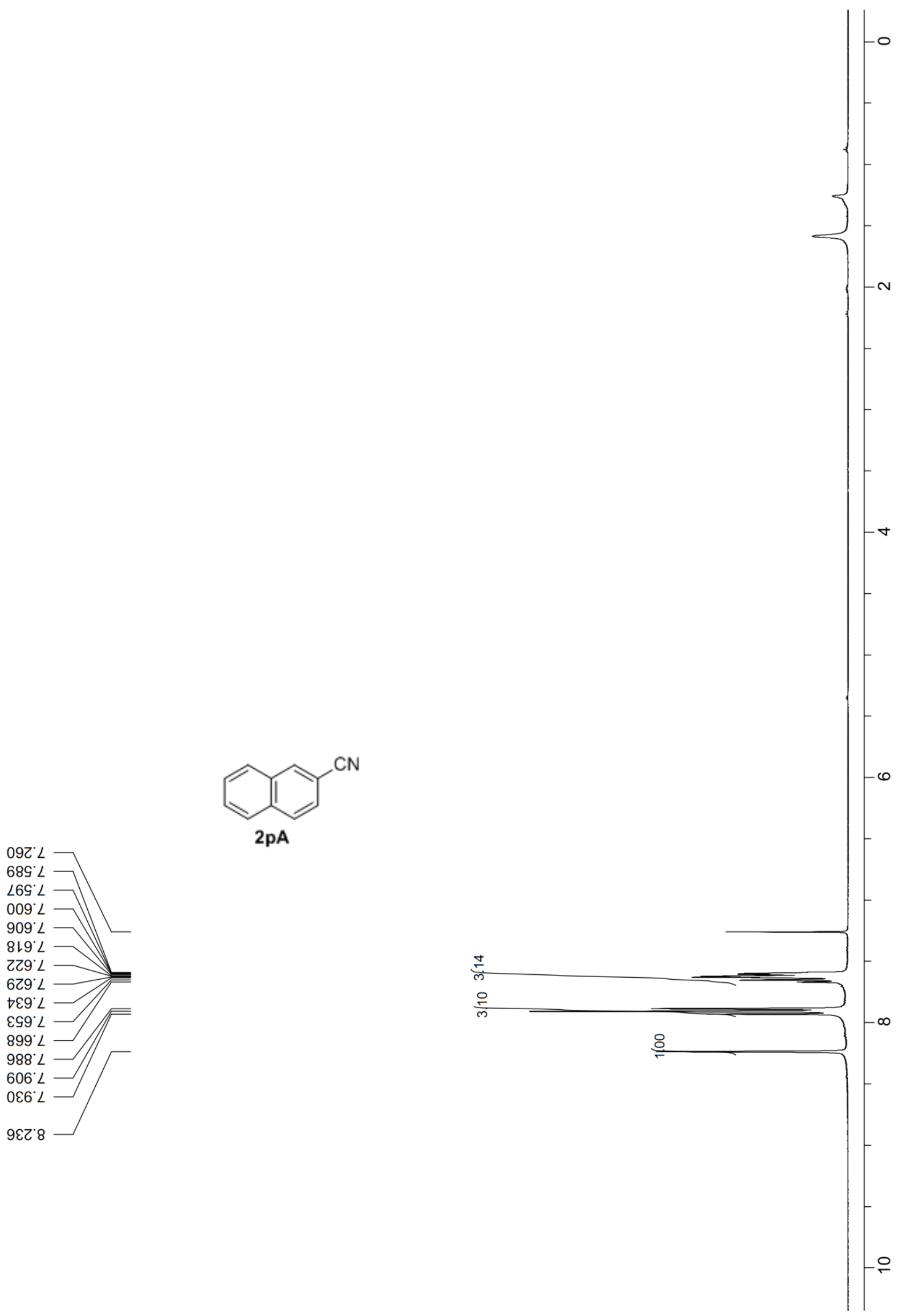


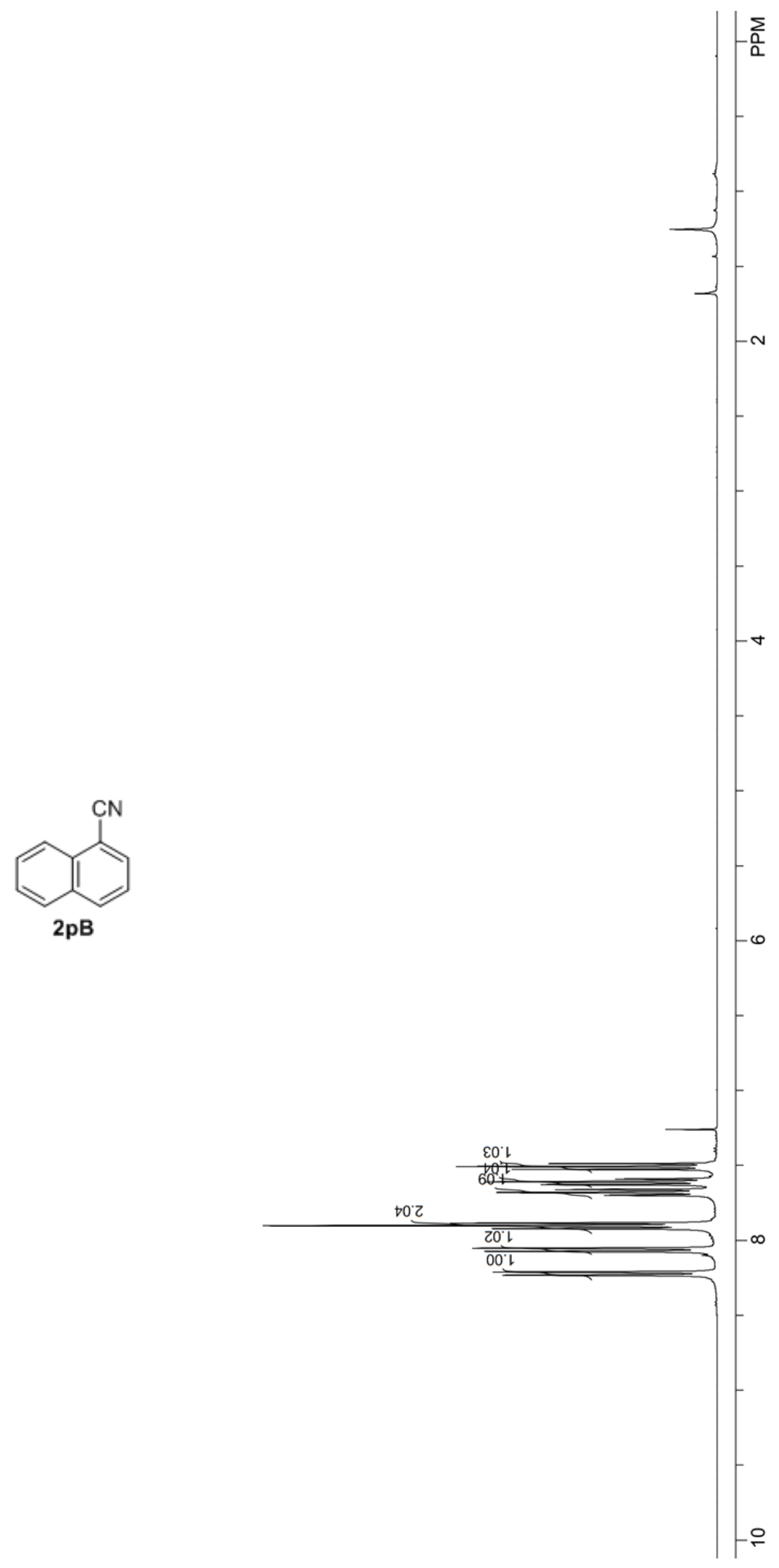



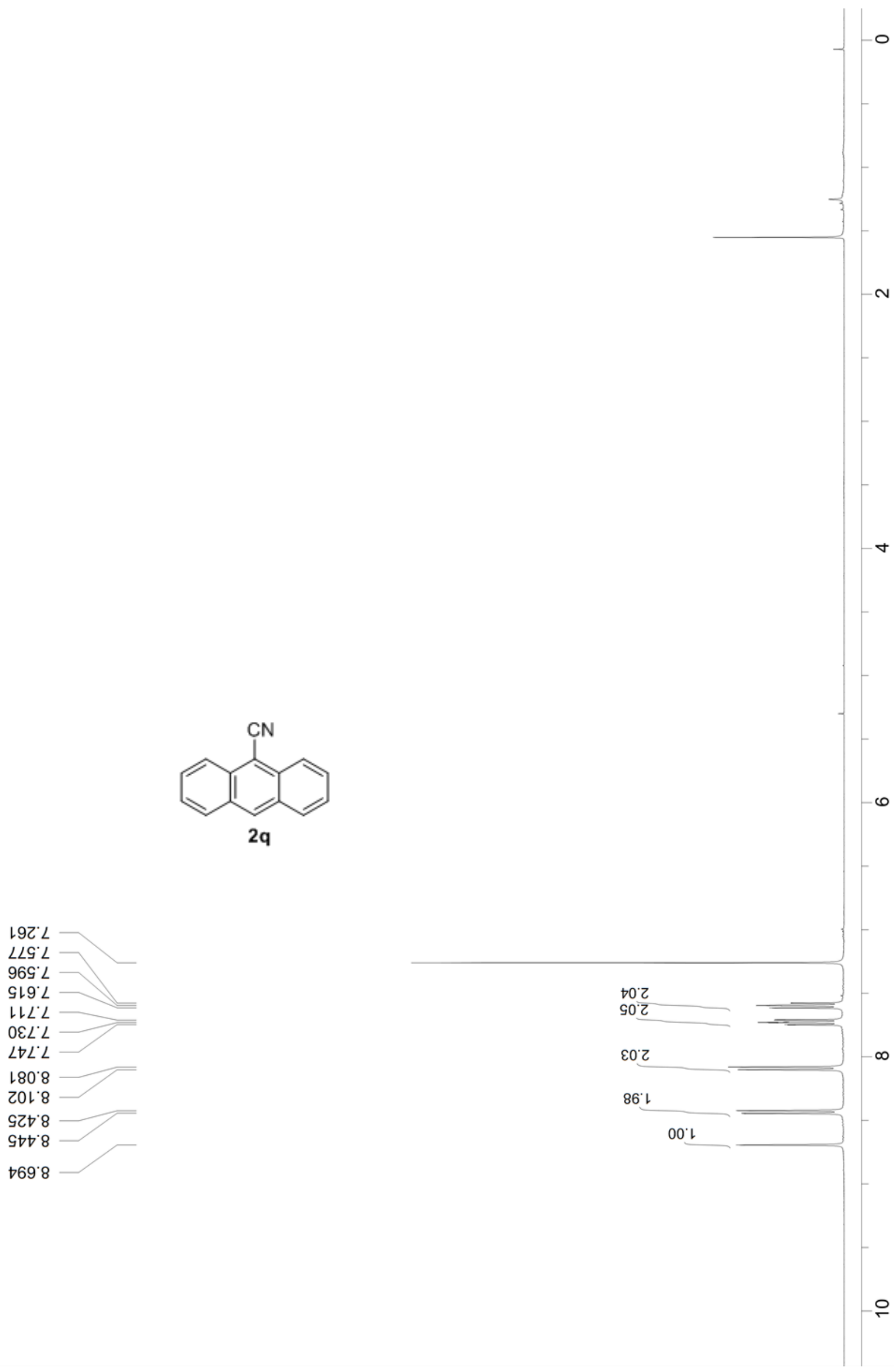

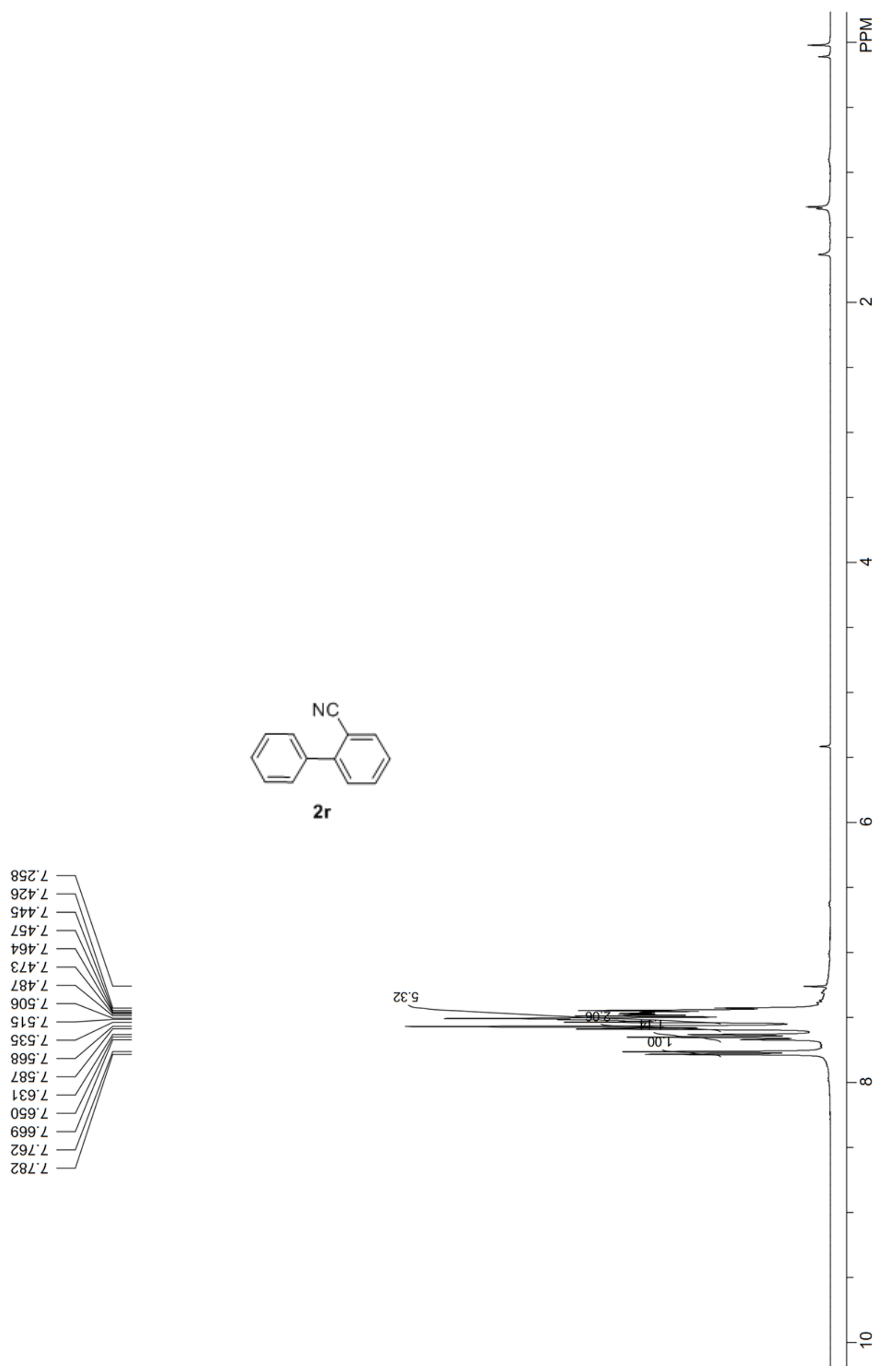
tLL $Z$

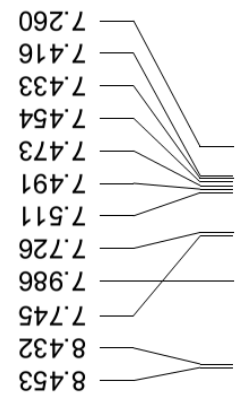

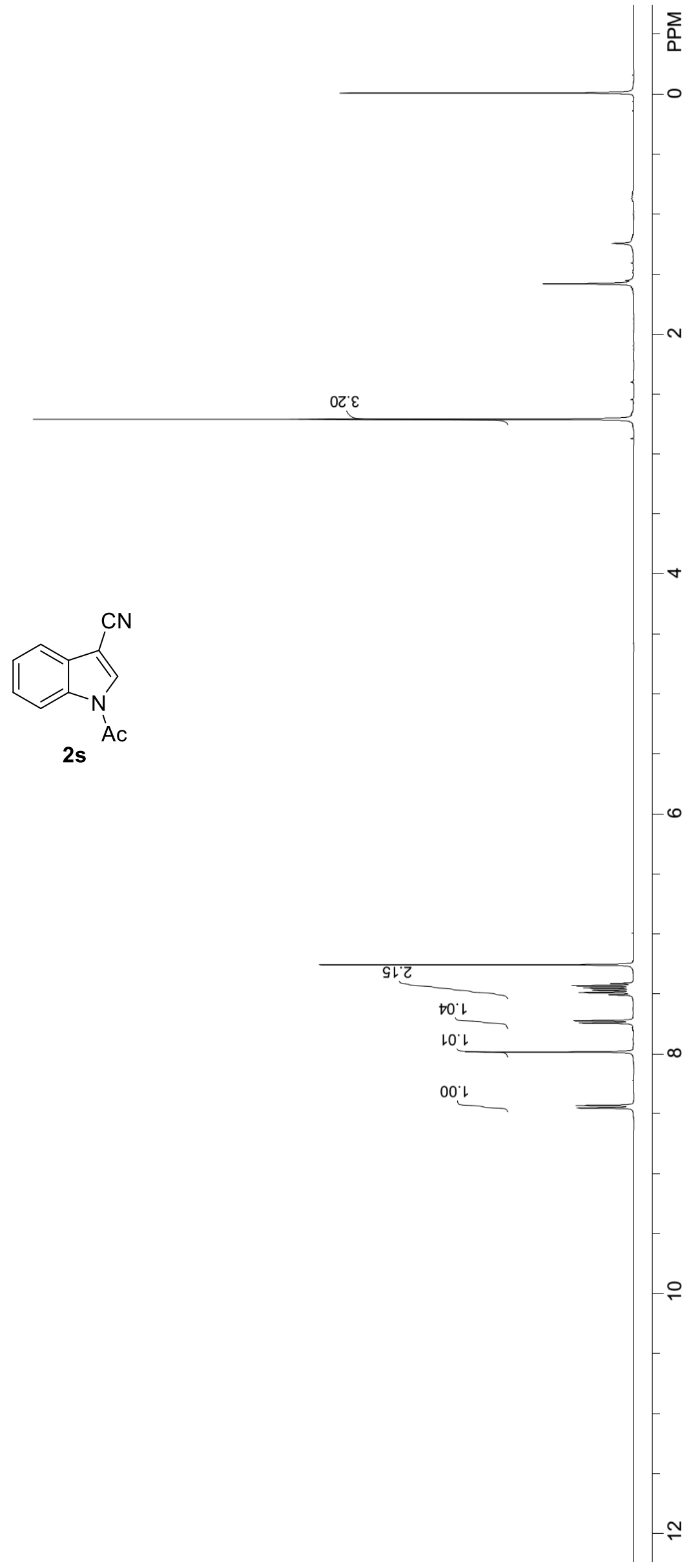




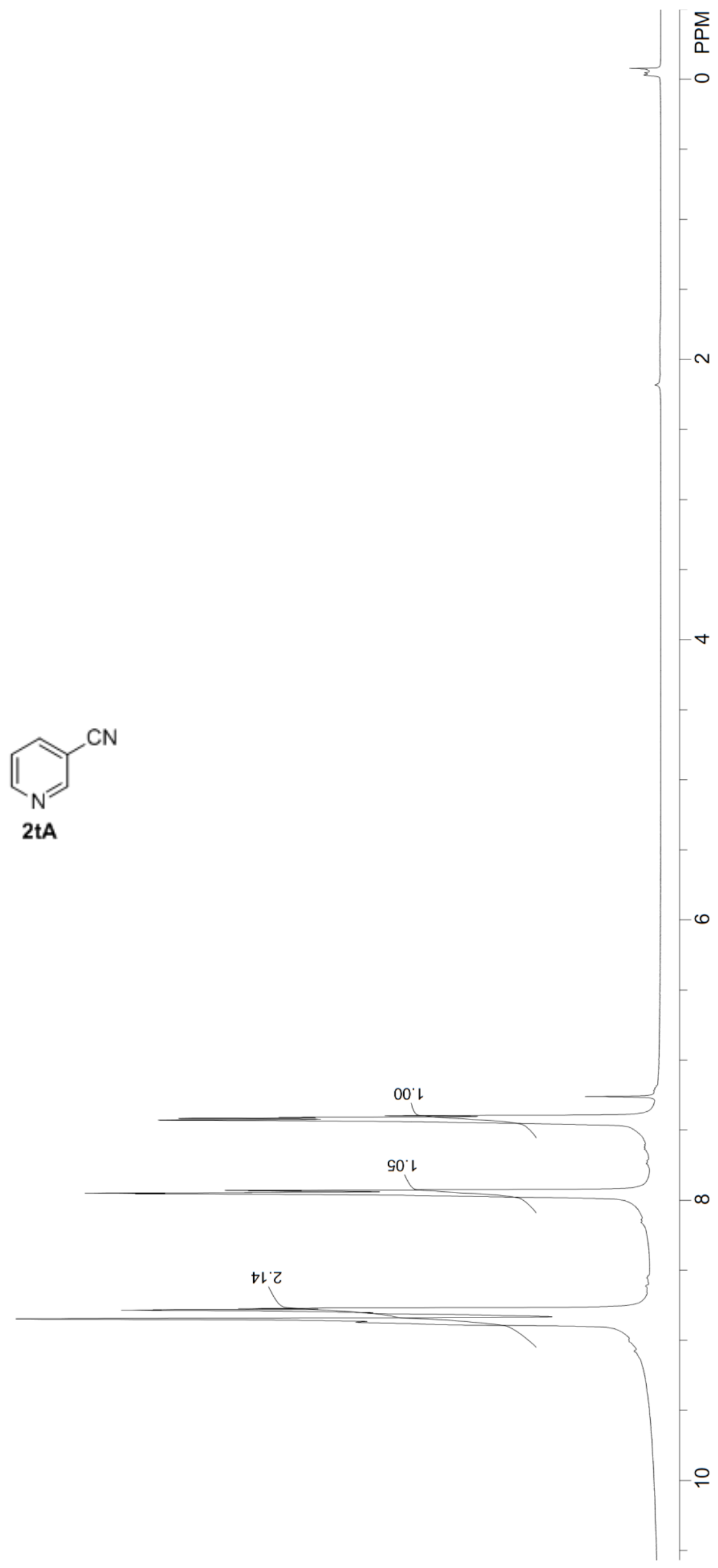



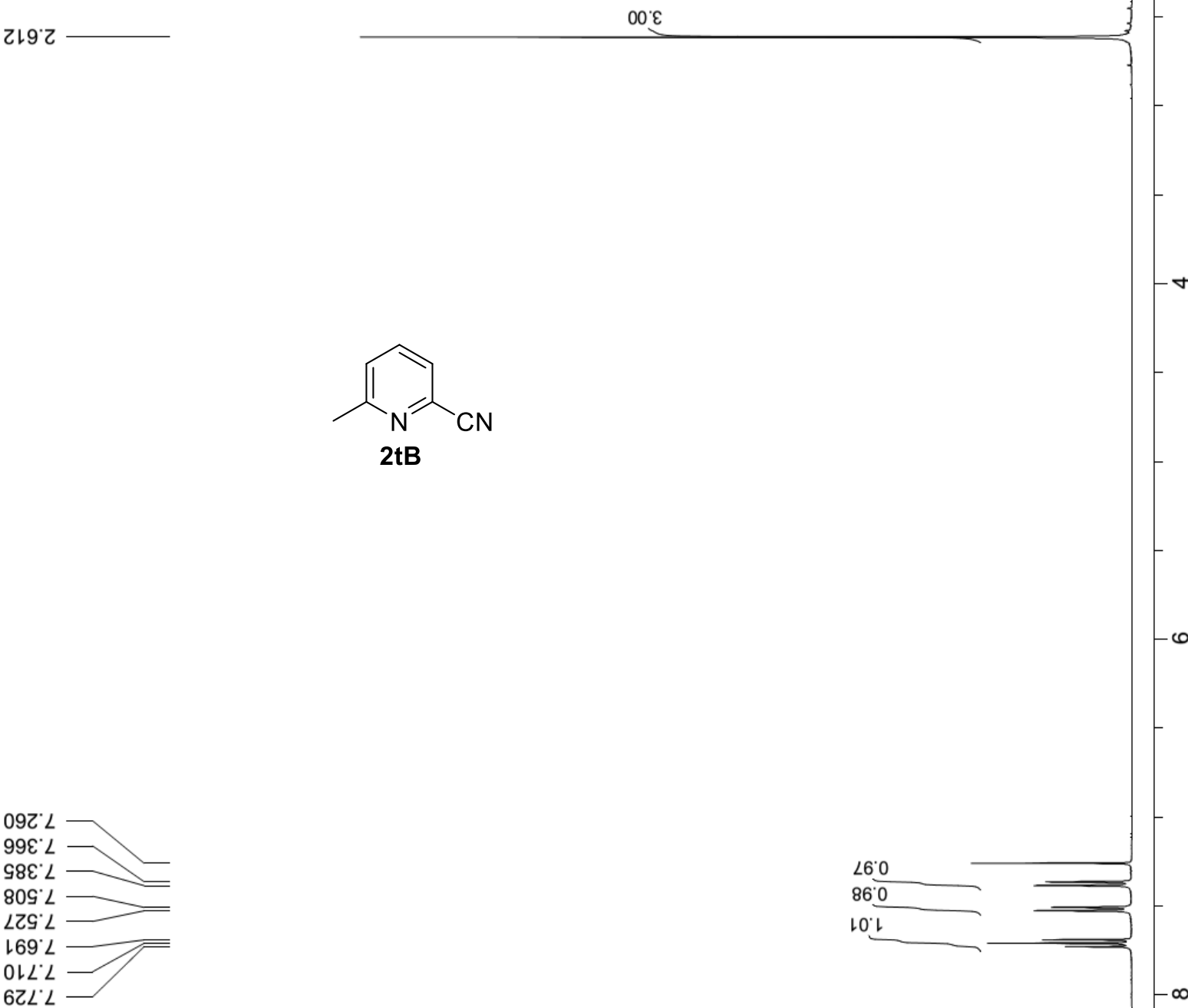
$9 \varepsilon 8^{\circ} \varepsilon$

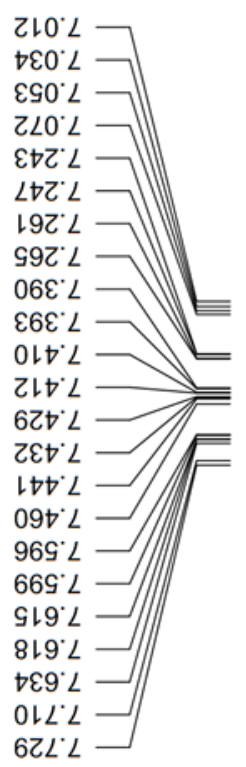

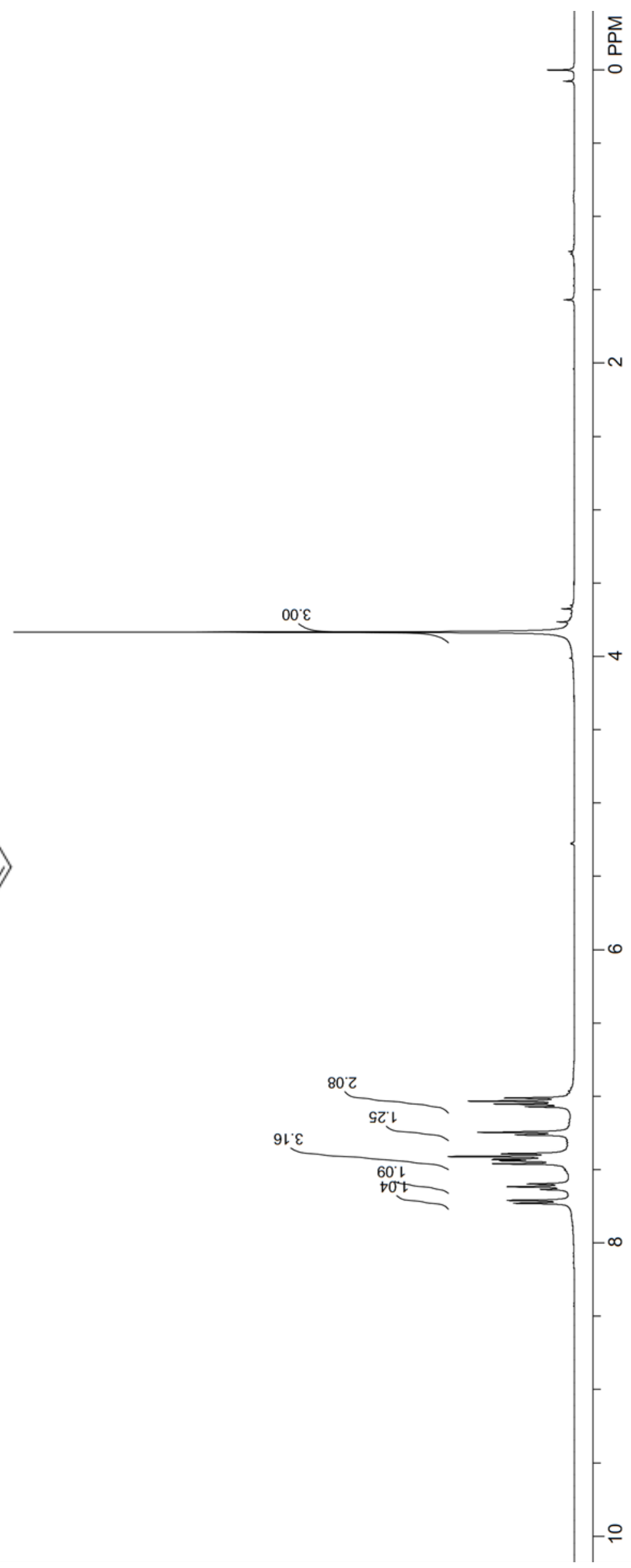



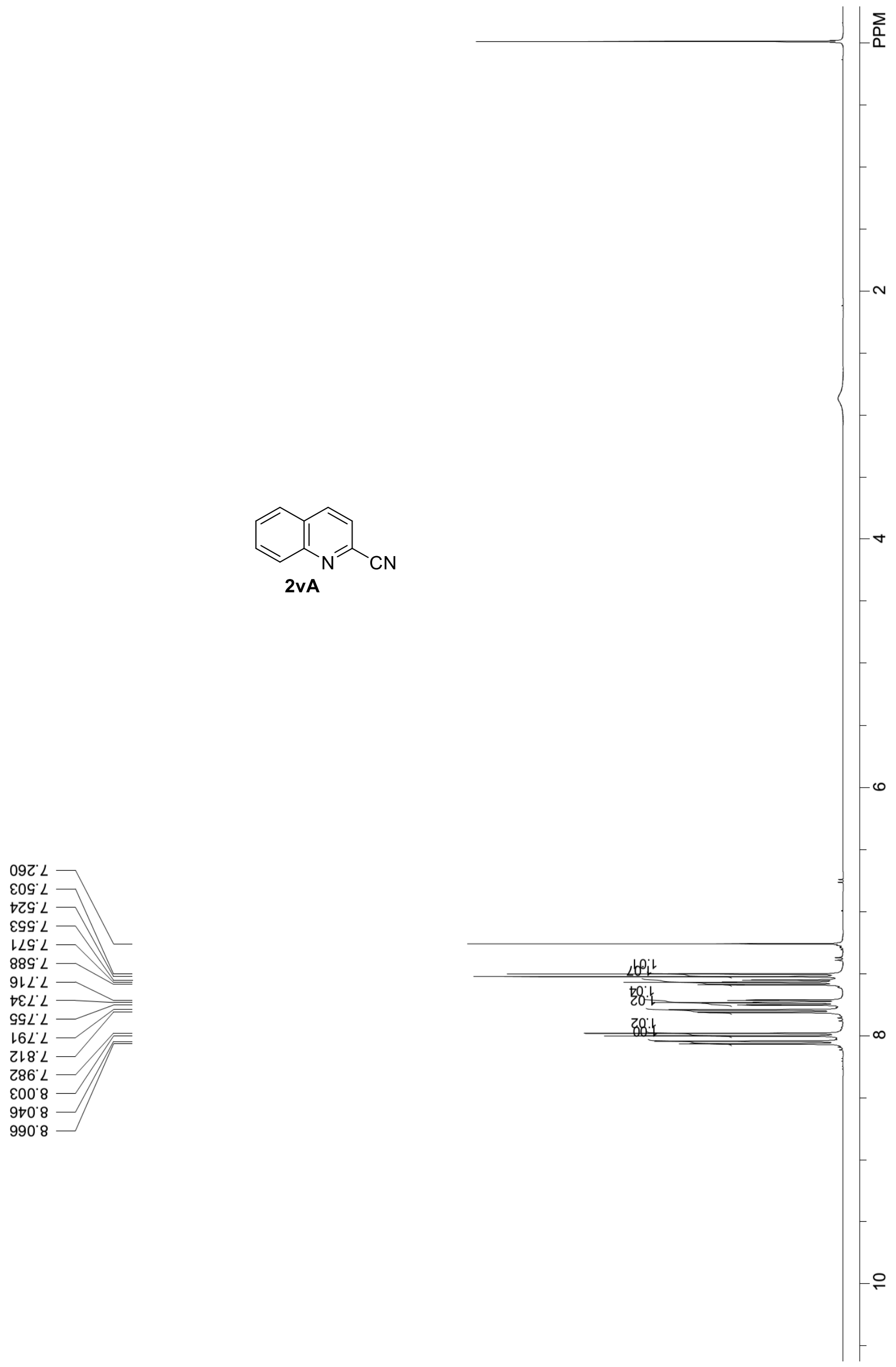


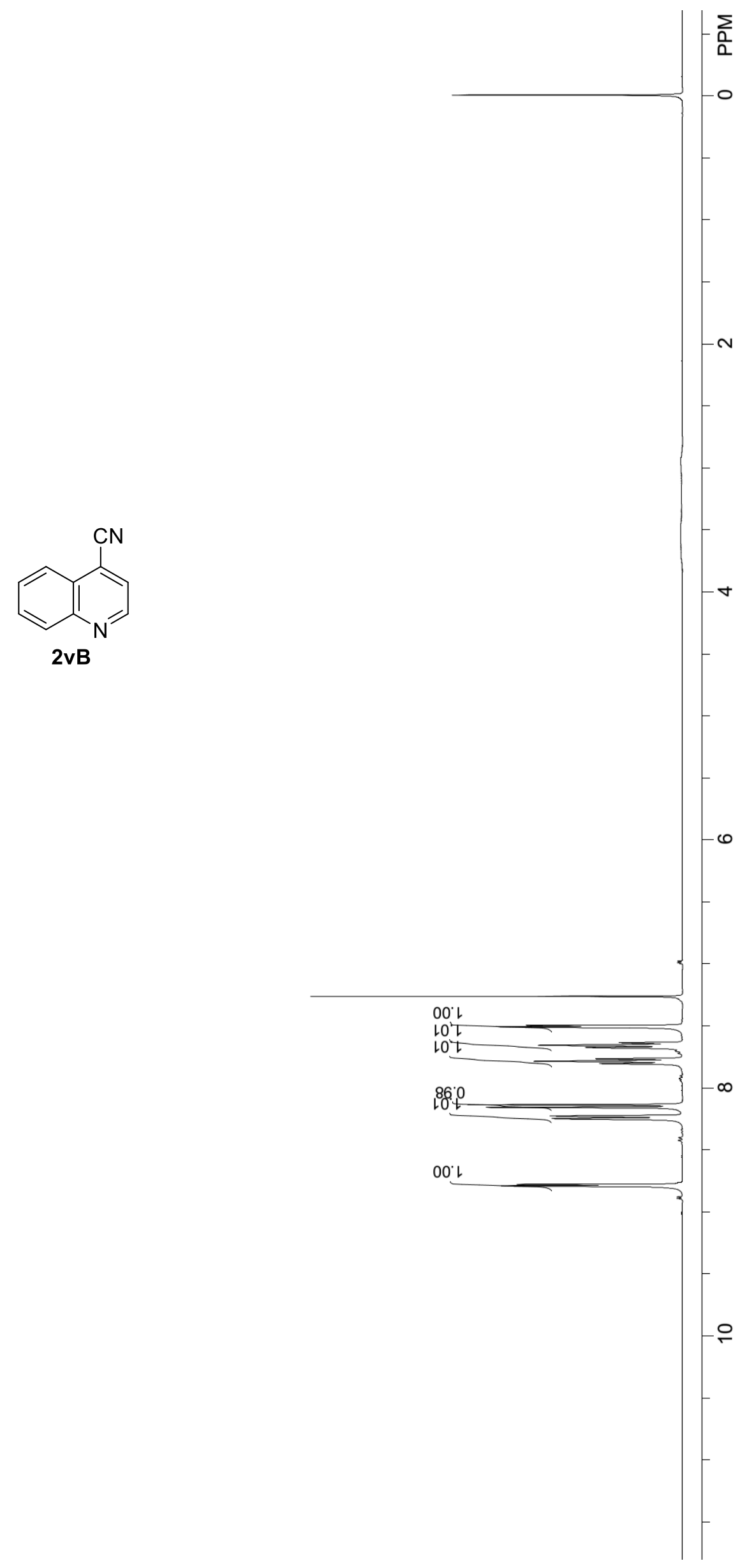




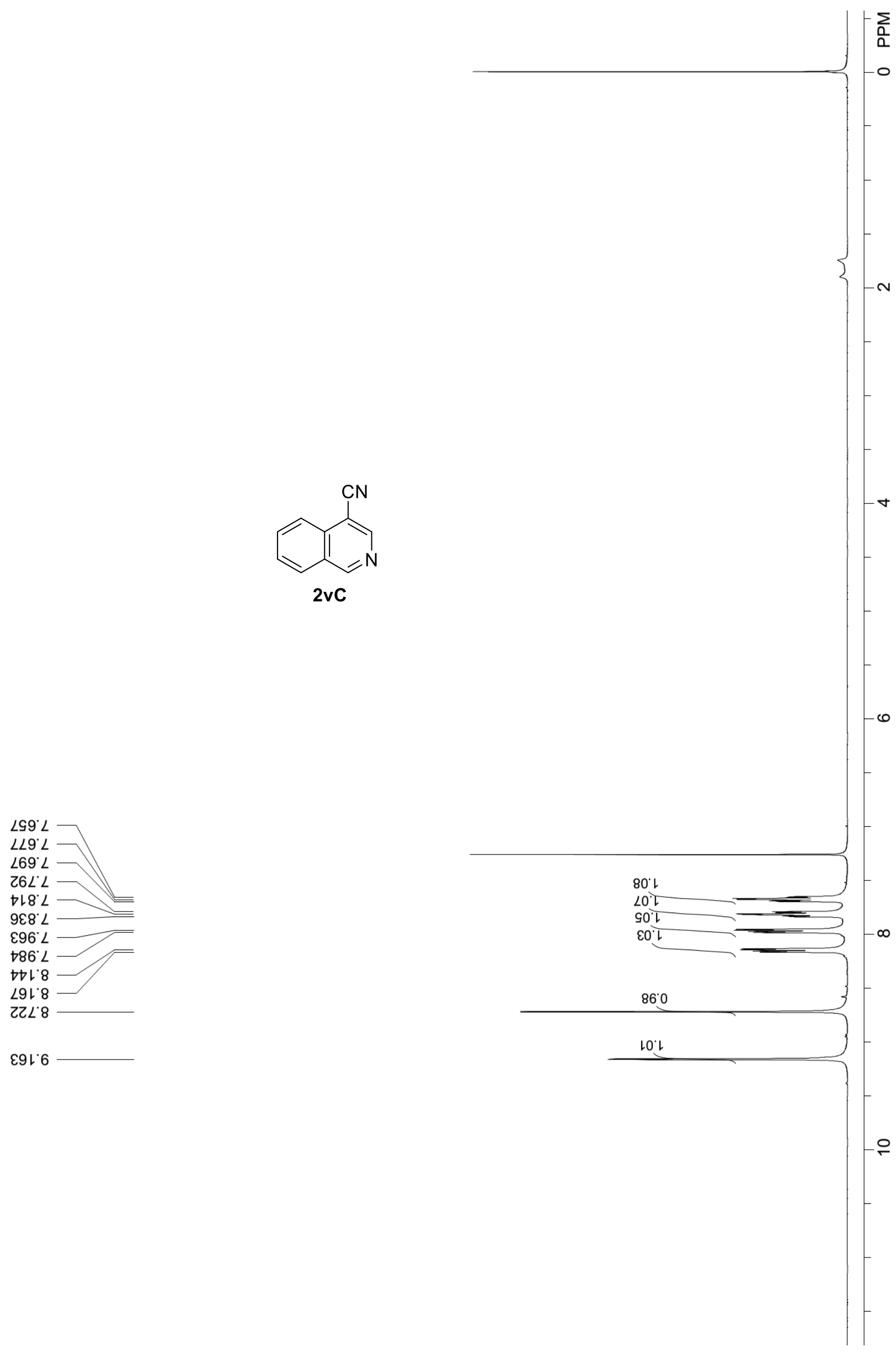




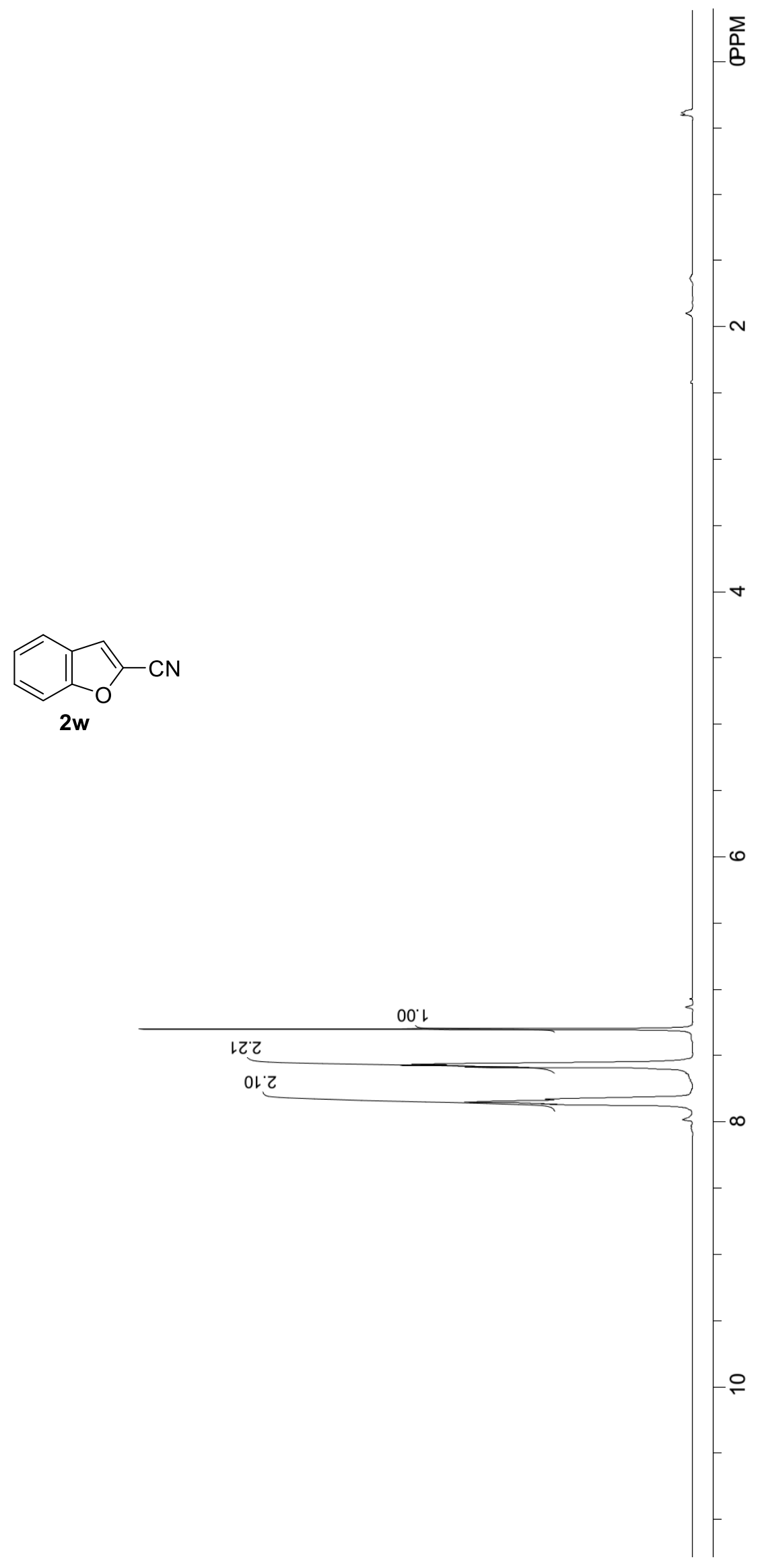

00E' $L$

999' $L$

6L9' $L$

E89. $L$

$689^{\circ} \mathrm{L}$

0ह8 $\mathrm{L}$

$878 . \mathrm{L}$

Z98 $\mathrm{L}$

$198^{\circ} \mathrm{L}$

$698^{\circ} \mathrm{L}$ 


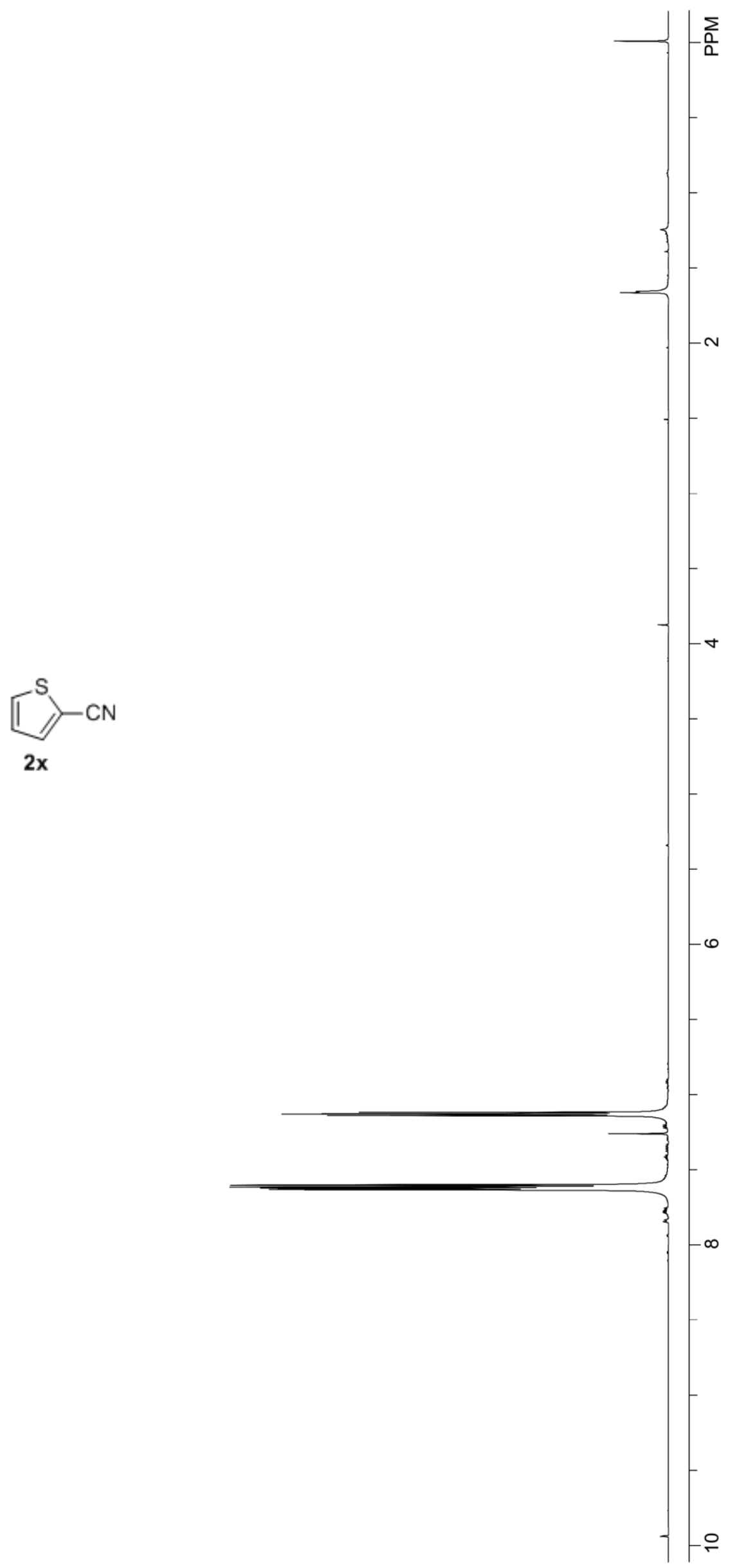




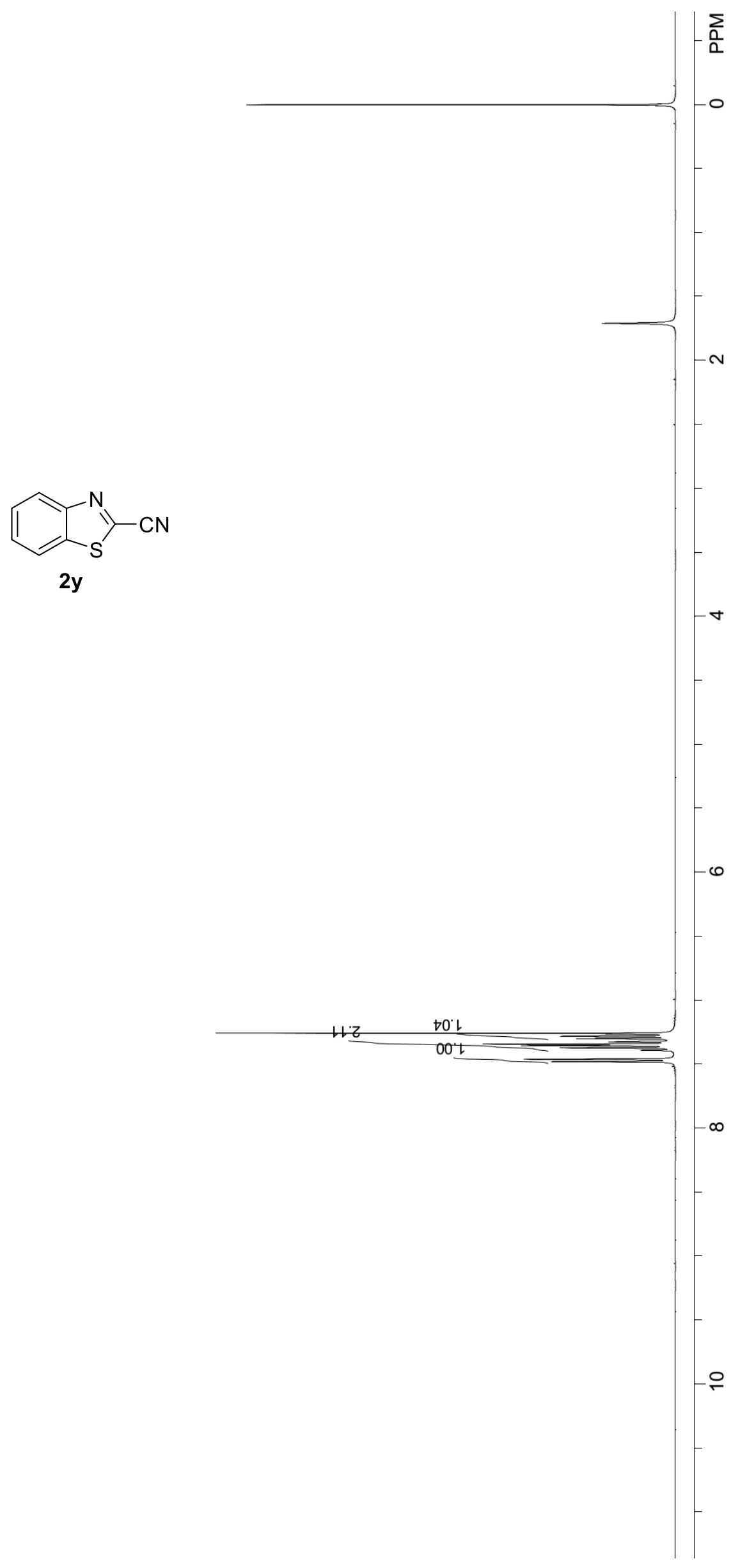


$700^{\circ} 0$

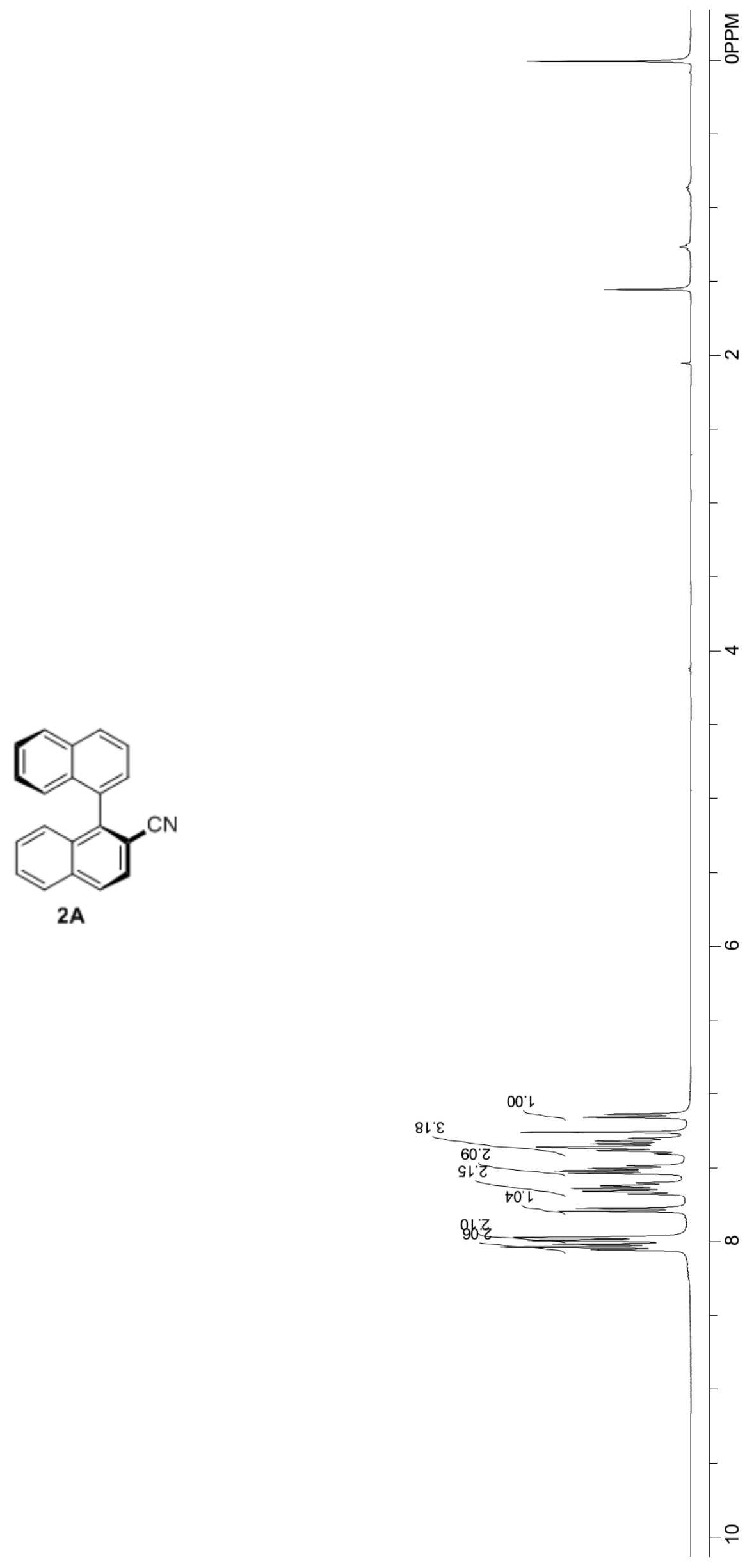

$8 \varepsilon L L$

6912

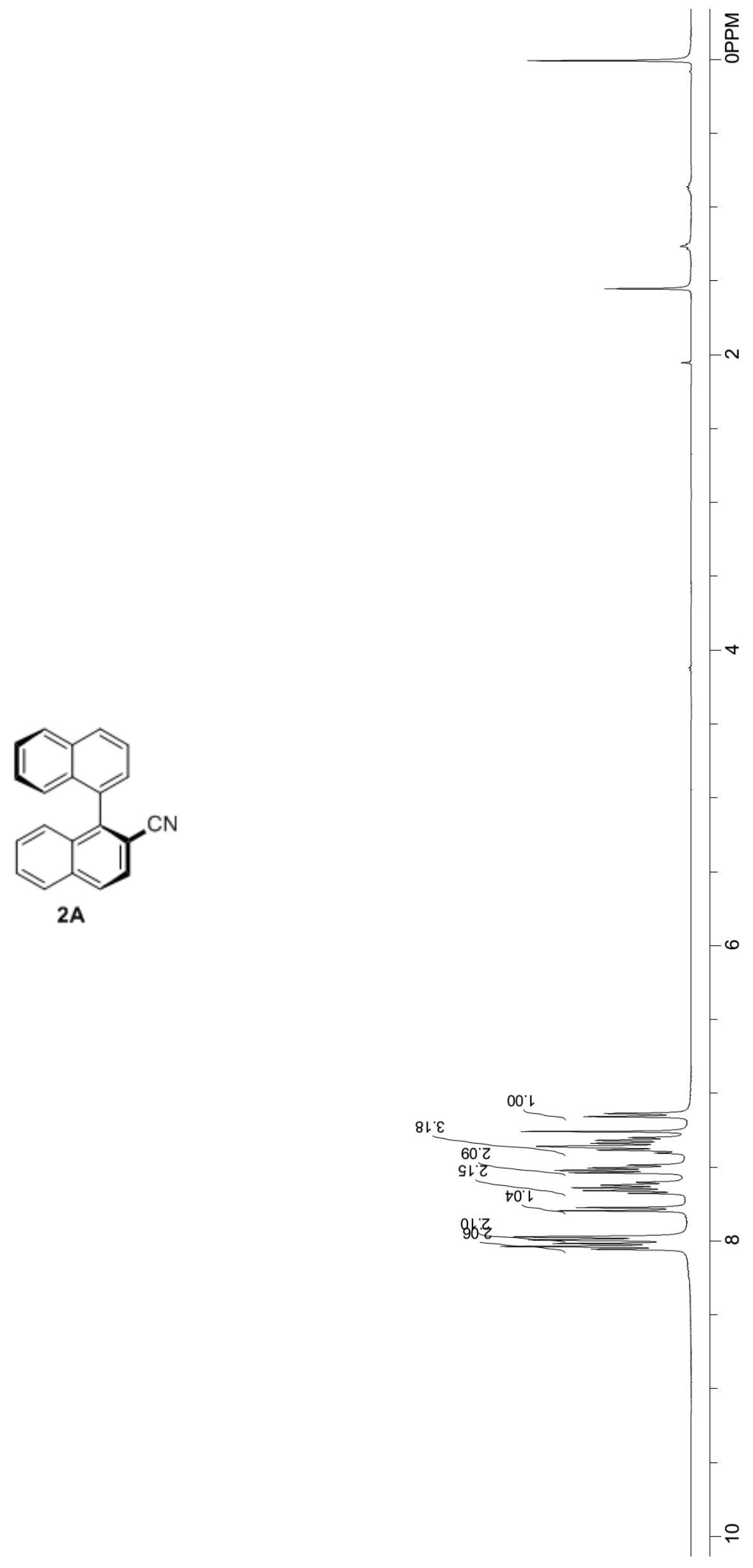

2A

092 L

ZОE' $L$

OZE $L$

$0 \sqcup \varepsilon^{\circ} L$

$09 \varepsilon^{\circ} L$

S8E. $L$

$90 t^{\circ} L$

$887^{\circ}$

$\angle O S^{\circ} \mathrm{L}$

EZG' $L$

$6 \varepsilon 9^{\circ} L$

$\rightarrow 09^{\circ} \mathrm{L}$

\&Z9 $L$

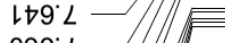

$099^{\circ} \mathrm{L}$

$6 \angle 9^{\circ} \mathrm{L}-$

SLL L

$\angle 6 L L$

$\nabla \angle 6 . \angle$

$766^{\circ} \mathrm{L}$

810.8

$6 \varepsilon 0.8$

$\angle 900^{\circ}$ 


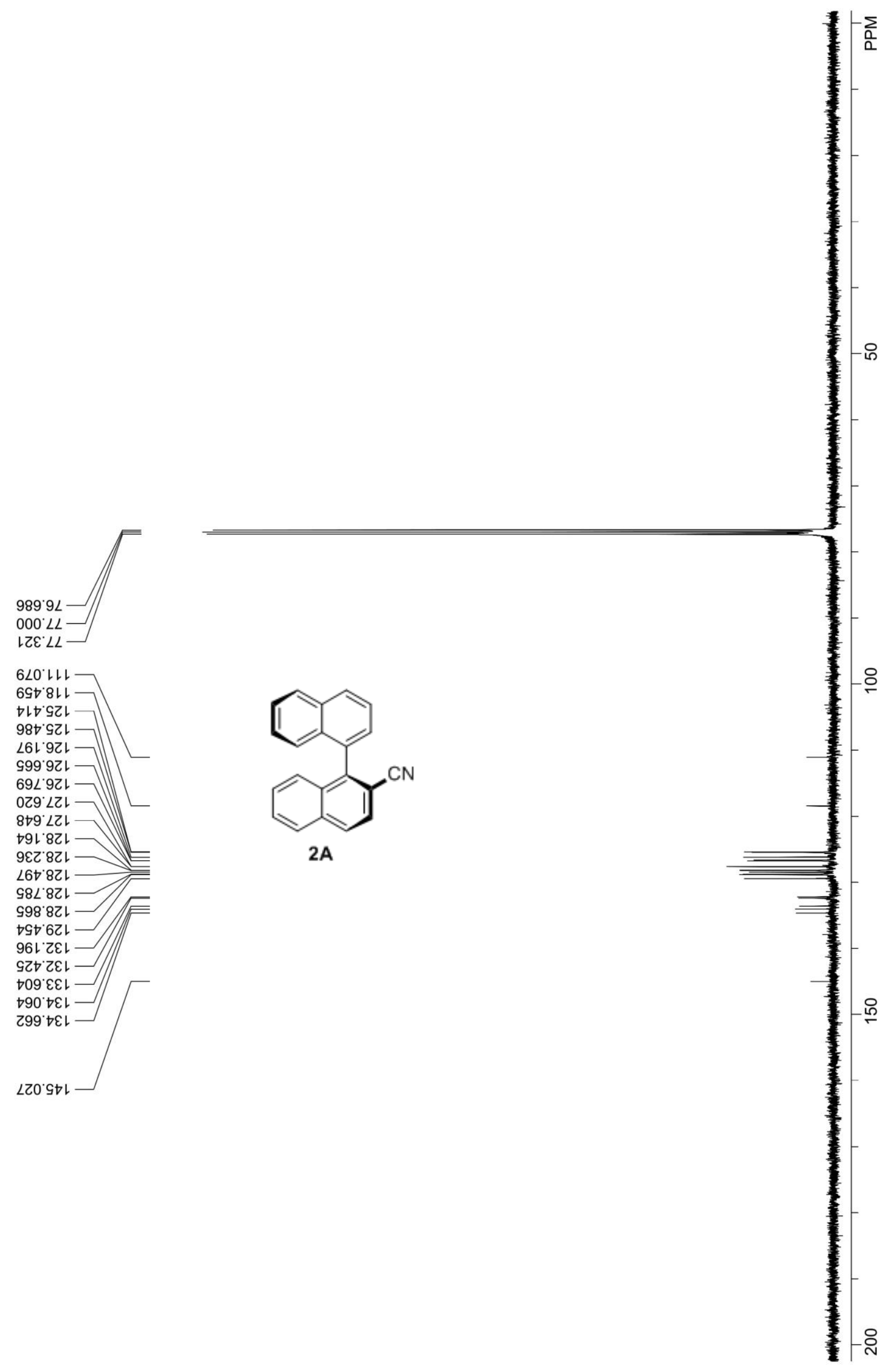


$900^{\circ} 0^{-}$

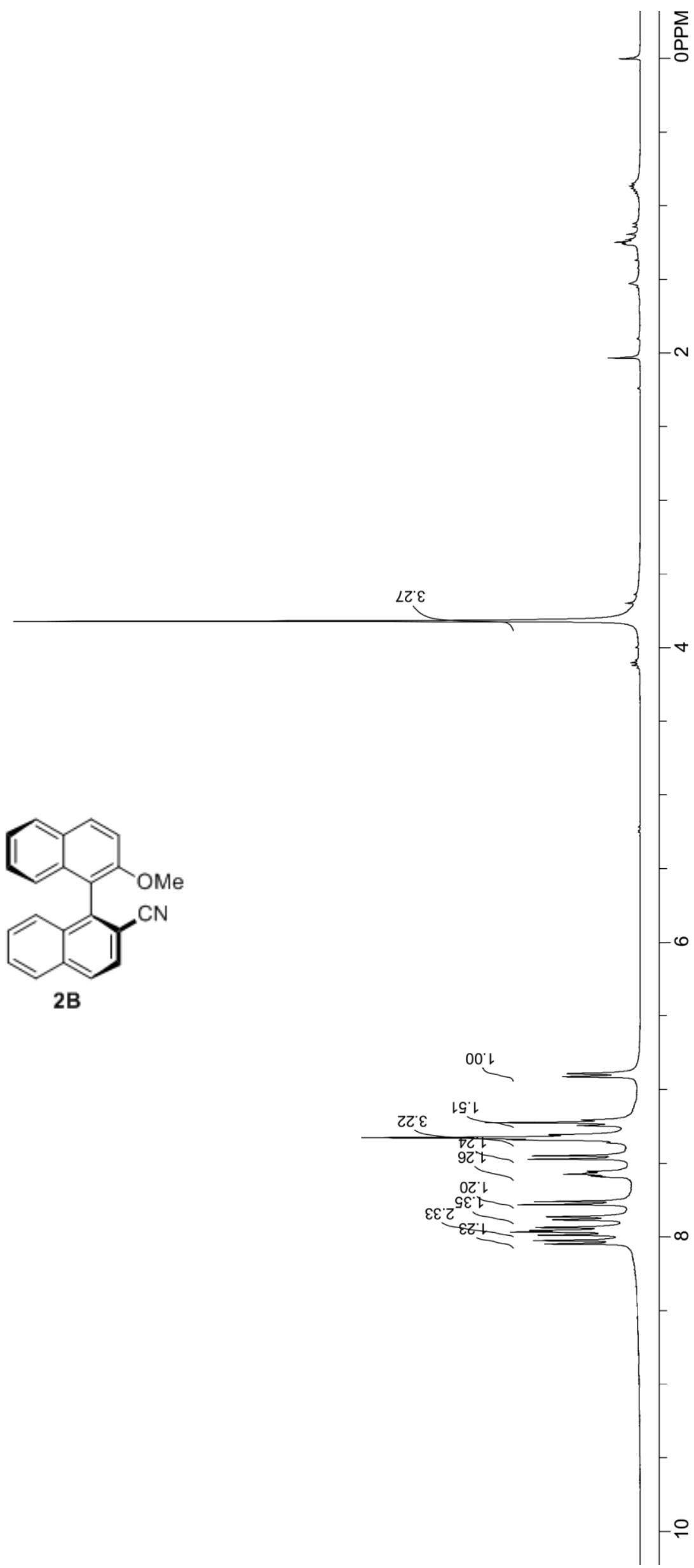

ع68.9

ㄴ.6.

$9 Z 2 \angle$

StZ

$\angle 0 \varepsilon^{\circ} \angle$

8ZE' $L$

$\downarrow \downarrow \varepsilon^{\circ} L$

ISt

$\nabla \angle t$

9SG

Z99' $\angle$

$0 \angle G^{\circ} \angle$

9LS

Z89' $\angle$

069' $L$

$96 S^{\circ} L$

$19 L ' L$

$\varepsilon 8 L^{\circ} L$

S98. $L$

G88.

$\angle E 6^{\circ}$

$896^{\circ} \mathrm{L}$

$896^{\circ} \mathrm{L}$

$686^{\circ}$

920.8

670.8 


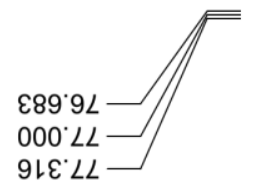

†08ルルー

†

$\angle 09^{\circ} 8 \mathrm{LL}$

6L9'8L

$\angle 8 L^{\prime} \varepsilon Z L$

$90 \varepsilon^{\circ} \triangleright Z \downarrow$

S06. 921

$780^{\circ} \mathrm{LZL}$

ZSI $\angle Z L$

$66 t^{\circ} \angle Z L$

$0 \angle L .8 Z L$

GIE'8ZL

LLG' $8 Z$ L

$688^{\circ} 8 \mathrm{ZL}$

เทレเレレ

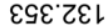

て6L・เレ

乙LE' $\varepsilon \varepsilon\llcorner$

ธะ८ ${ }^{\circ}\llcorner$

$\angle Z 8^{\circ} \downarrow G$
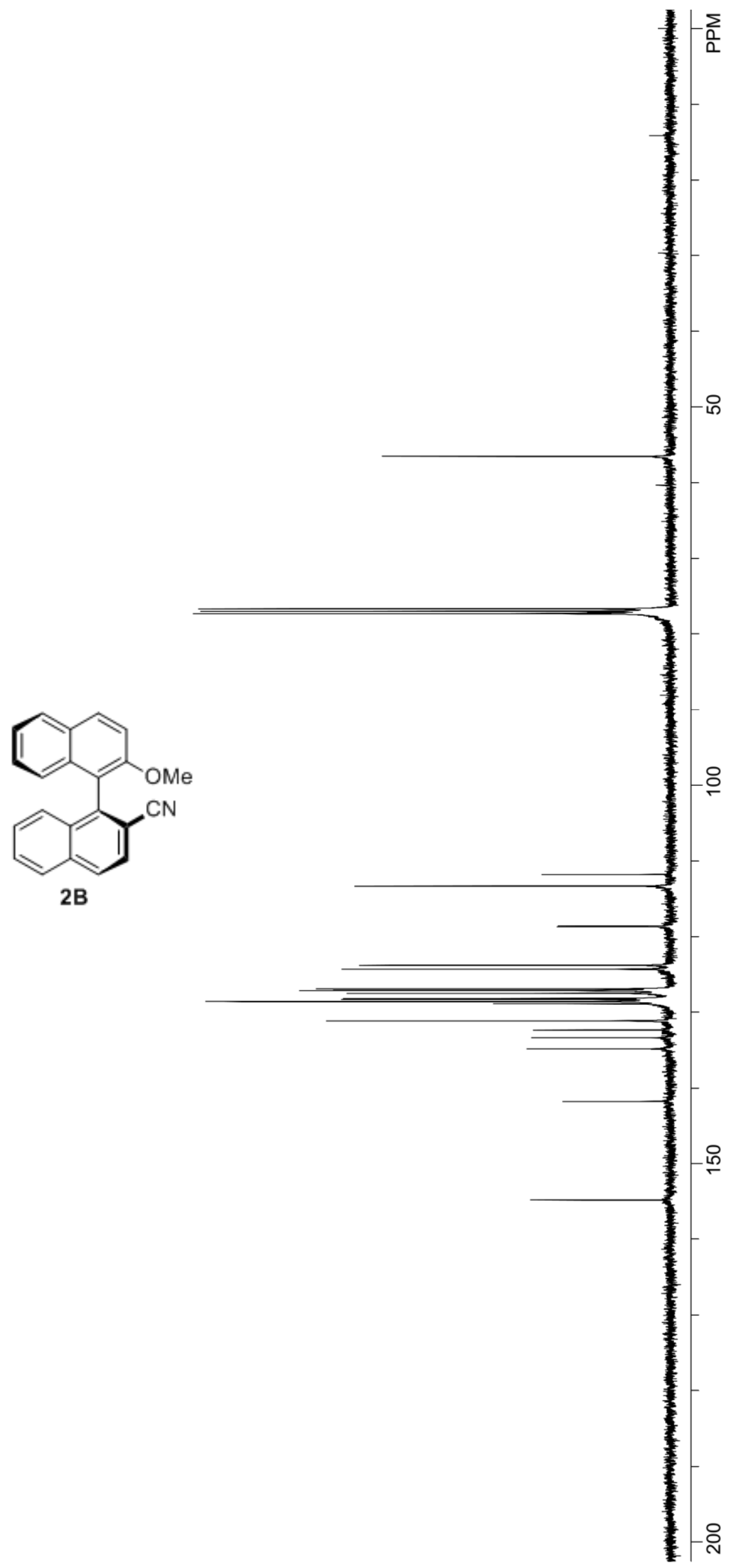
L6L'

$\angle t 0^{\circ} \mathrm{L}$

$690^{\circ} \mathrm{L}$

$092 \angle$

OLE $L$

6ZE L

$8 \nabla \varepsilon^{\circ} L$

$6 L \varepsilon^{\circ} L$

$\nabla 6 \varepsilon^{\circ} L$

SLT $L$

$\forall \angle D^{\circ} L$

$109^{\circ}$

$909^{\circ} \mathrm{L}$

$129 . \mathrm{L}$

$\angle Z 9^{\circ} \mathrm{L}$

$9 \varepsilon 9^{\circ} L$

$1+9.2$

L6L'L

ZL8. $L$

$596^{\circ} \mathrm{L}$

$986^{\circ} \mathrm{L}$

乙ह००

¿९0

$\checkmark \angle 0^{\circ}$

9608

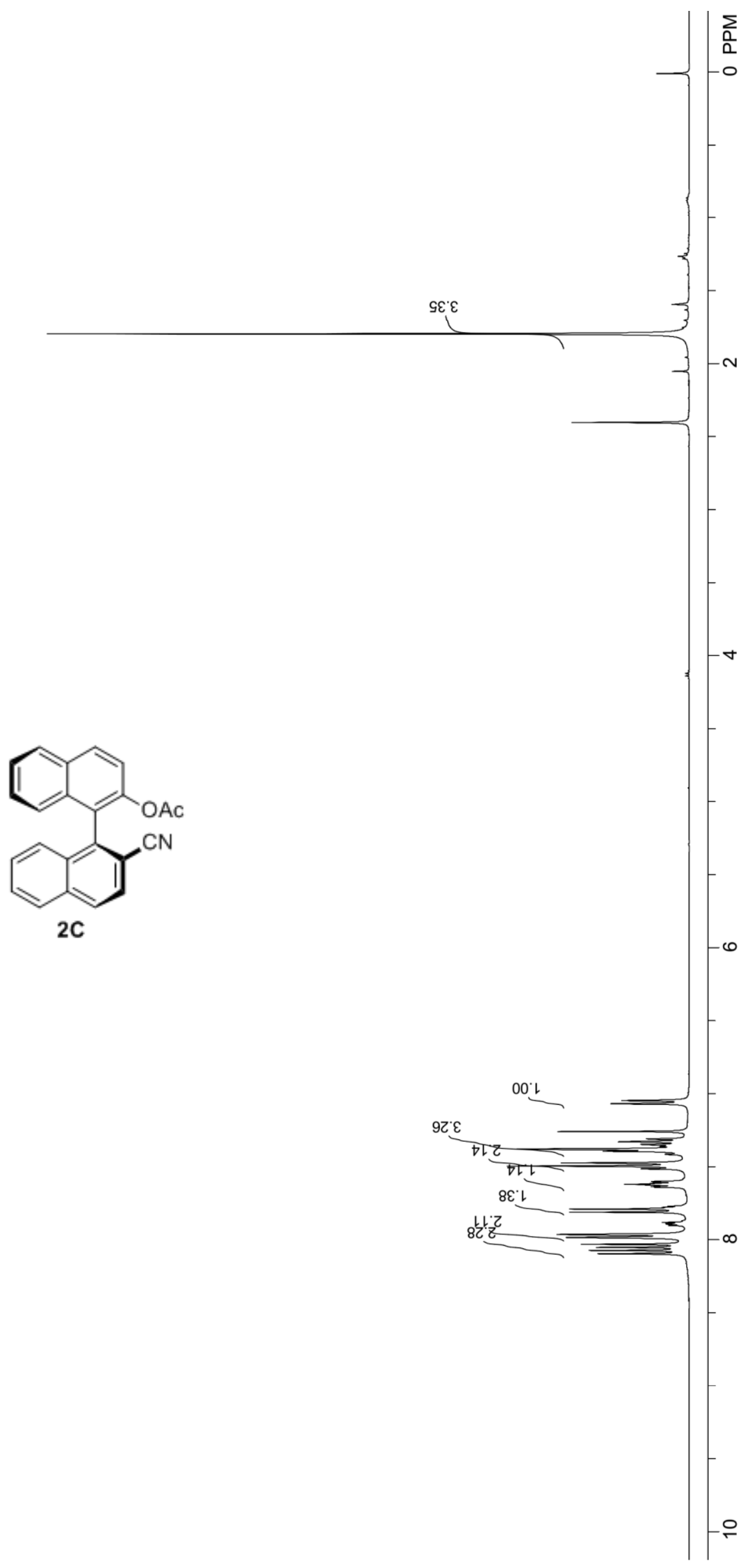



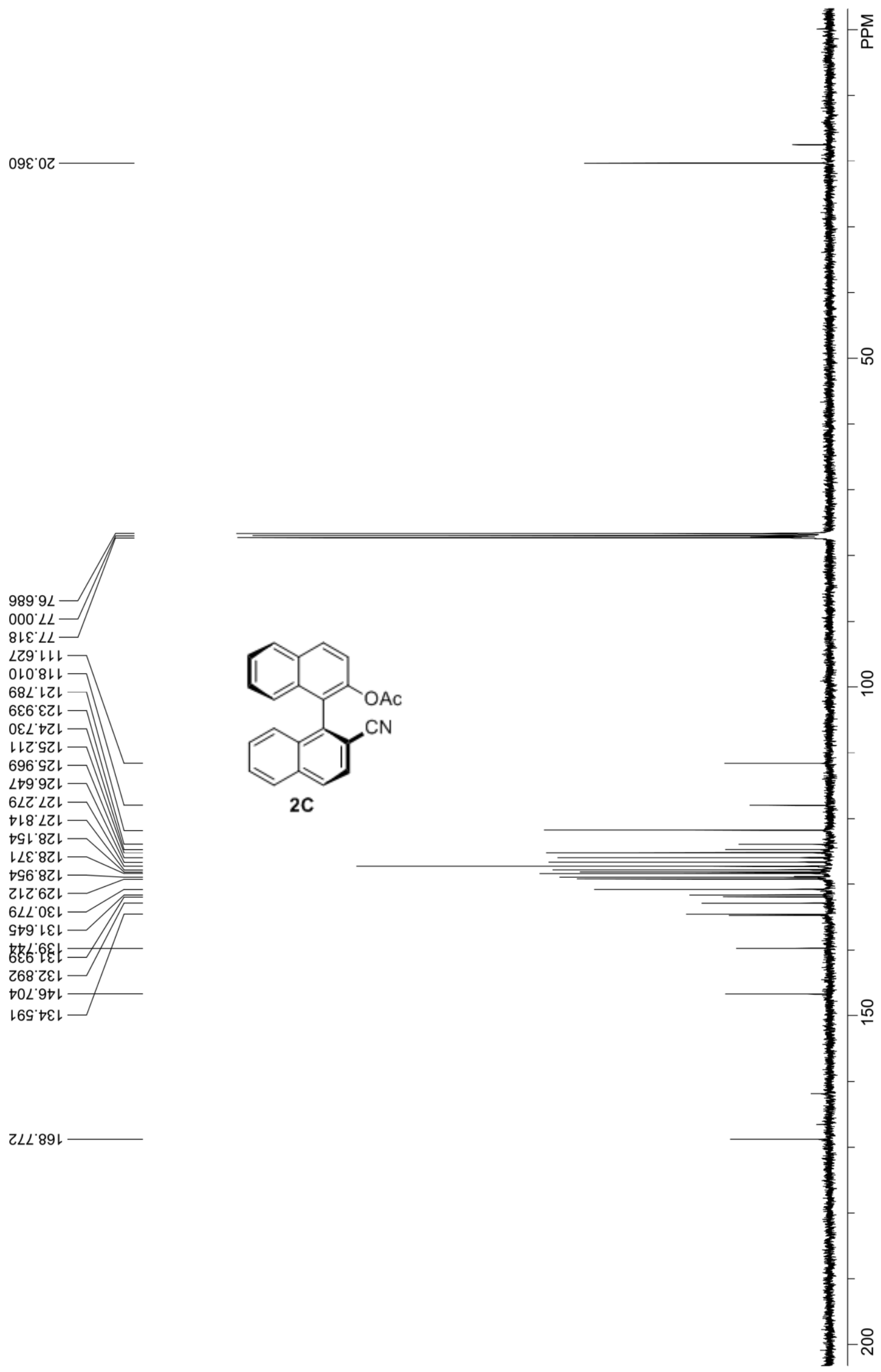

$000^{\circ} \mathrm{L}$

OLO'BL

68Lにて

レ乙GZレ

เ७9.

$6 \angle Z$ ' $L Z$

th 22

ZLL'89L 
6ยt

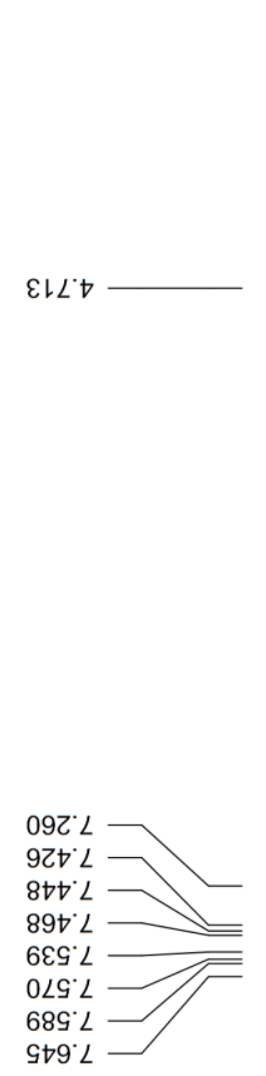<smiles>N#Cc1cccc(CO)c1</smiles>

002
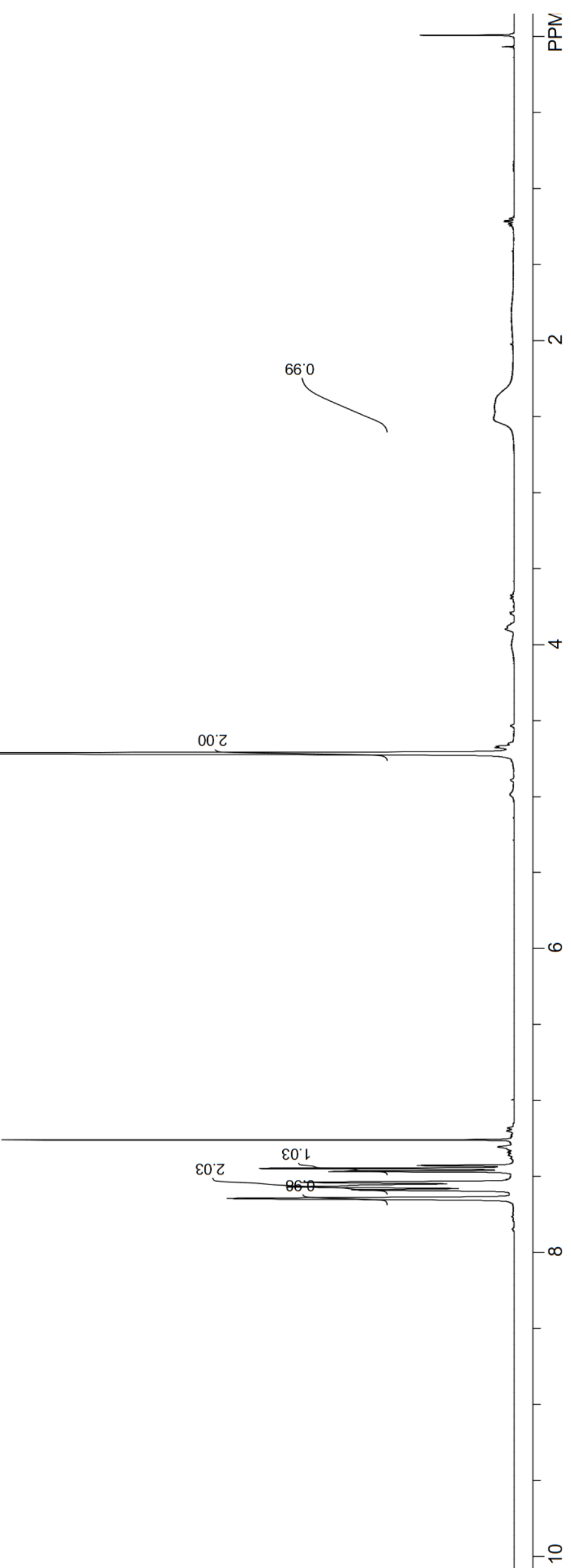

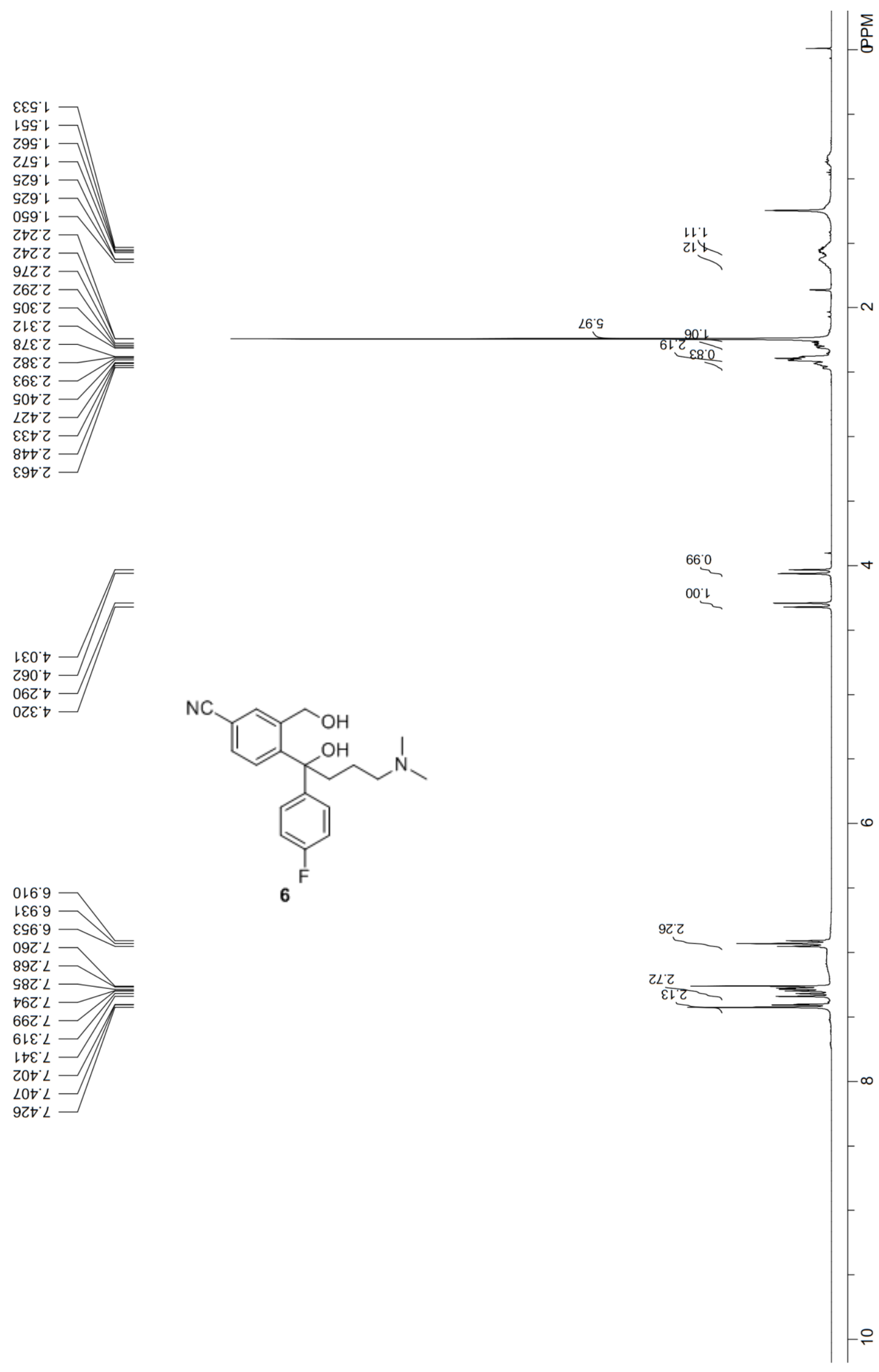
$\varepsilon 69^{\circ} \varepsilon t$ 189'tt

$\nabla 6 L^{\circ}$

乙๕8: 69

$\varepsilon 0 Z^{\circ} \triangleright 9$

$\varepsilon 0 L \cdot 9 L$

$\angle L O^{\circ} \angle L$

$0 \triangleright \varepsilon \cdot L$

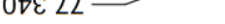

เง゙๋เレレ
เ99เレレ

$\angle Z Z\llcorner Z L$ IEG $\angle Z L$

$809^{\circ} \angle Z L$

乙El. $8 \mathrm{~L}$

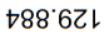

$\dashv 8 Z^{\circ} \varsigma \varepsilon \downarrow$

ZZ6'てヤ

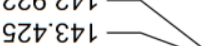

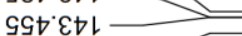

S08 tto

เOZ.09เ $6 \varepsilon 99^{\circ} \mathrm{Zl}$

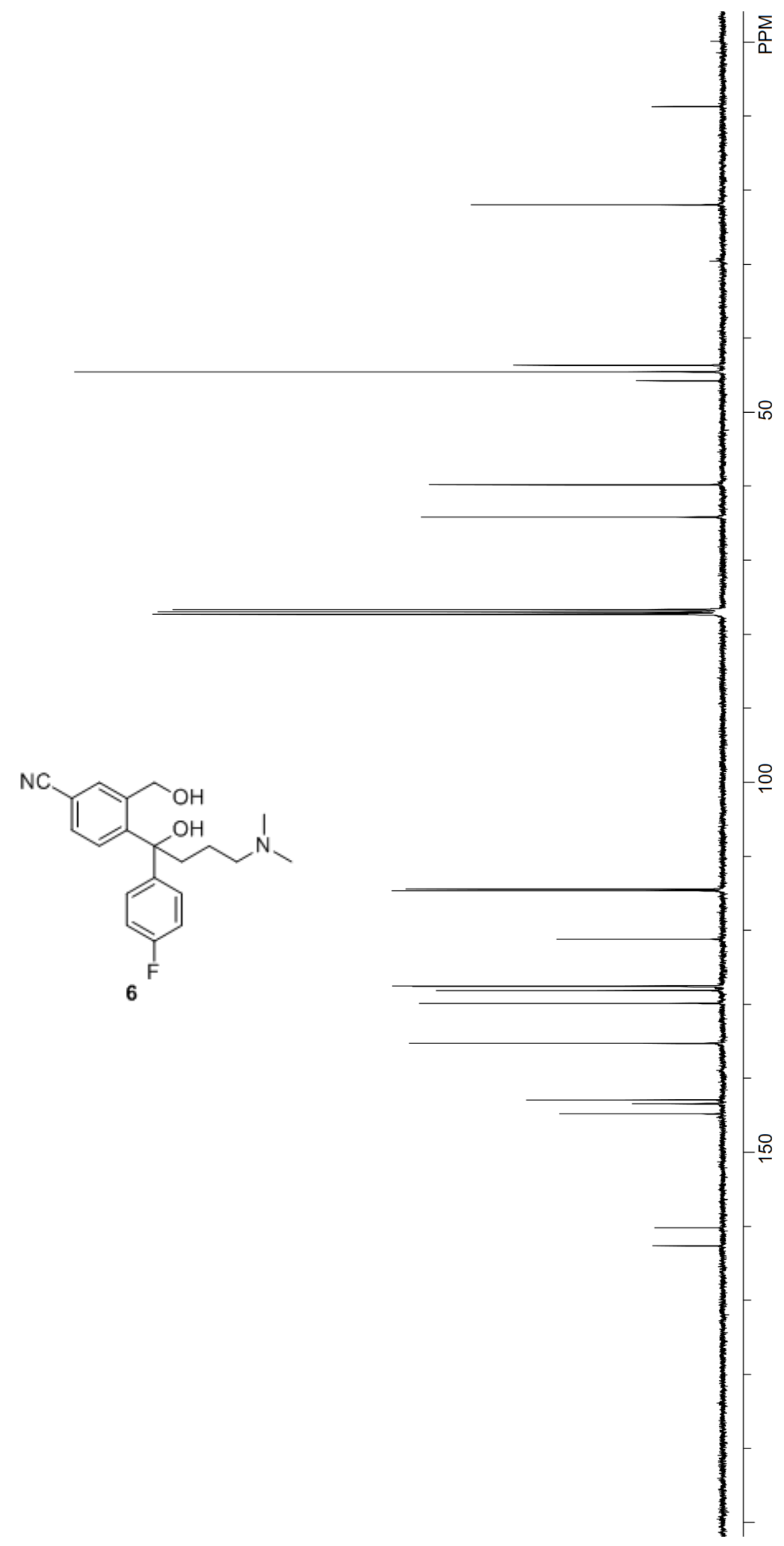



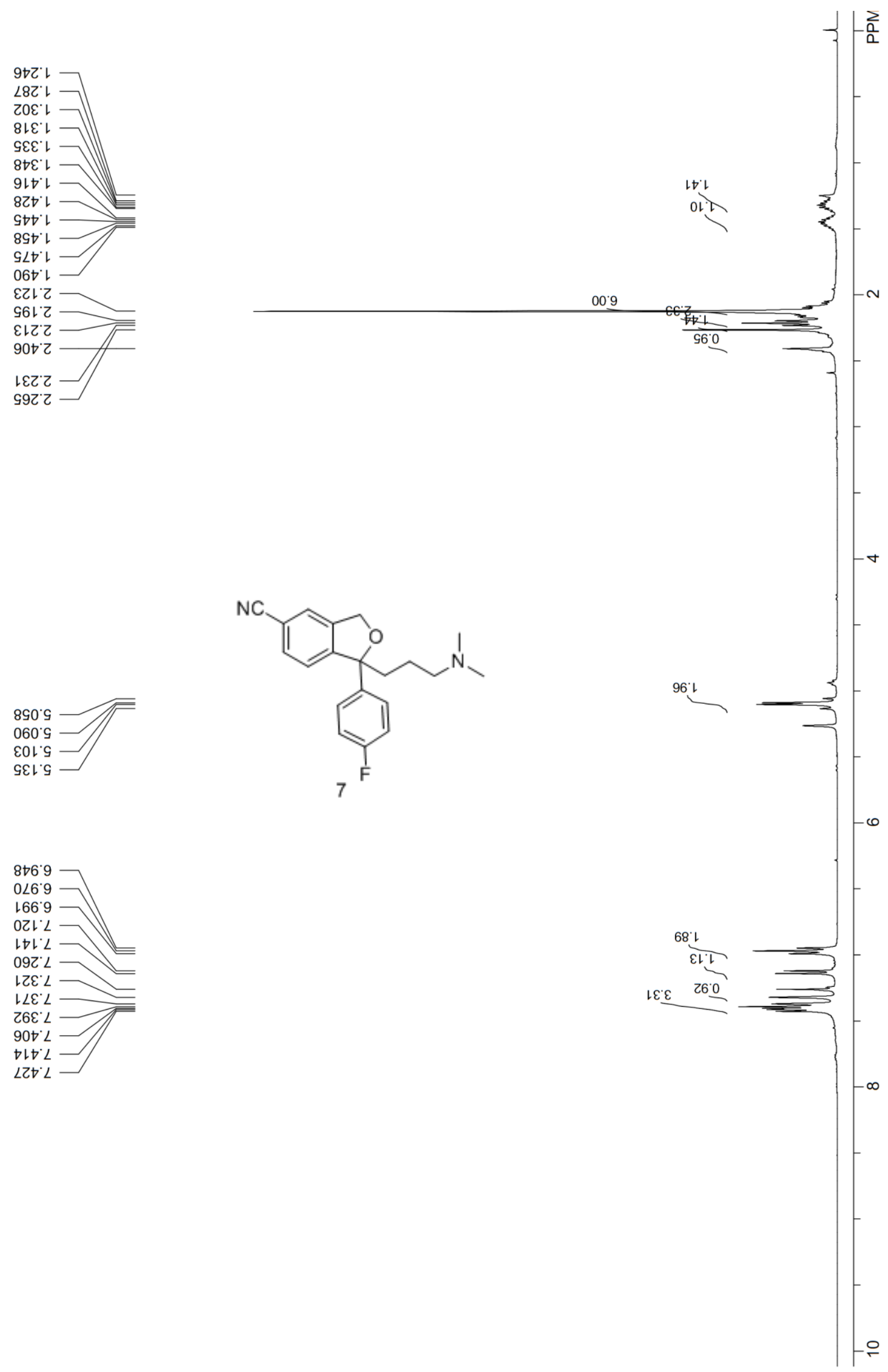
Chrom Type: HPLC Channel : 1

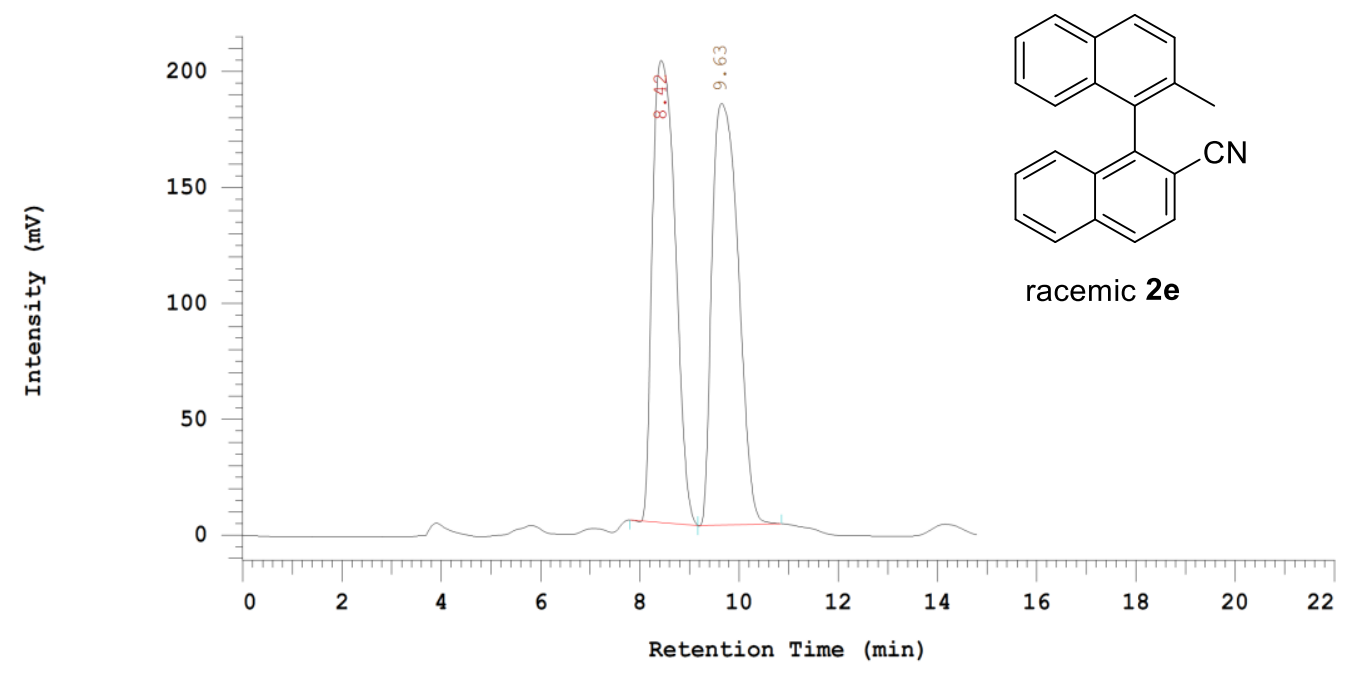

\begin{tabular}{ccccc} 
No. & RT & Area & Conc 1 & BC \\
\hline 1 & 8.42 & 6416324 & 48.788 & MC \\
2 & 9.63 & 6735053 & 51.212 & MC \\
\hline & & 13151377 & 100.000 &
\end{tabular}

Peak rejection level: 0

Chrom Type: HPLC Channel : 1

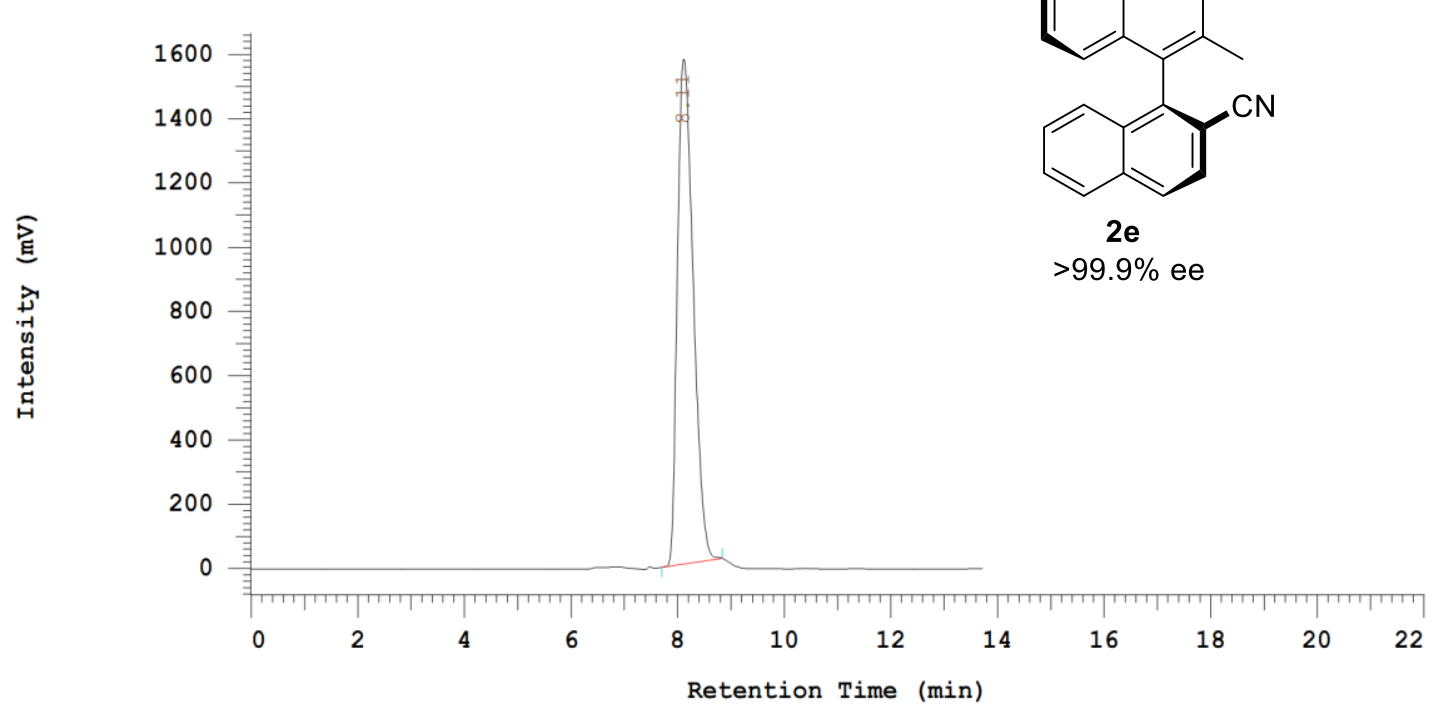

\begin{tabular}{ccccc} 
No. & RT & Area & Conc 1 & BC \\
\hline 1 & 8.11 & 32235075 & 100.000 & MC \\
\hline & 32235075 & 100.000 & \\
\hline
\end{tabular}

Peak rejection level: 0 
Chrom Type: HPLC Channel : 1

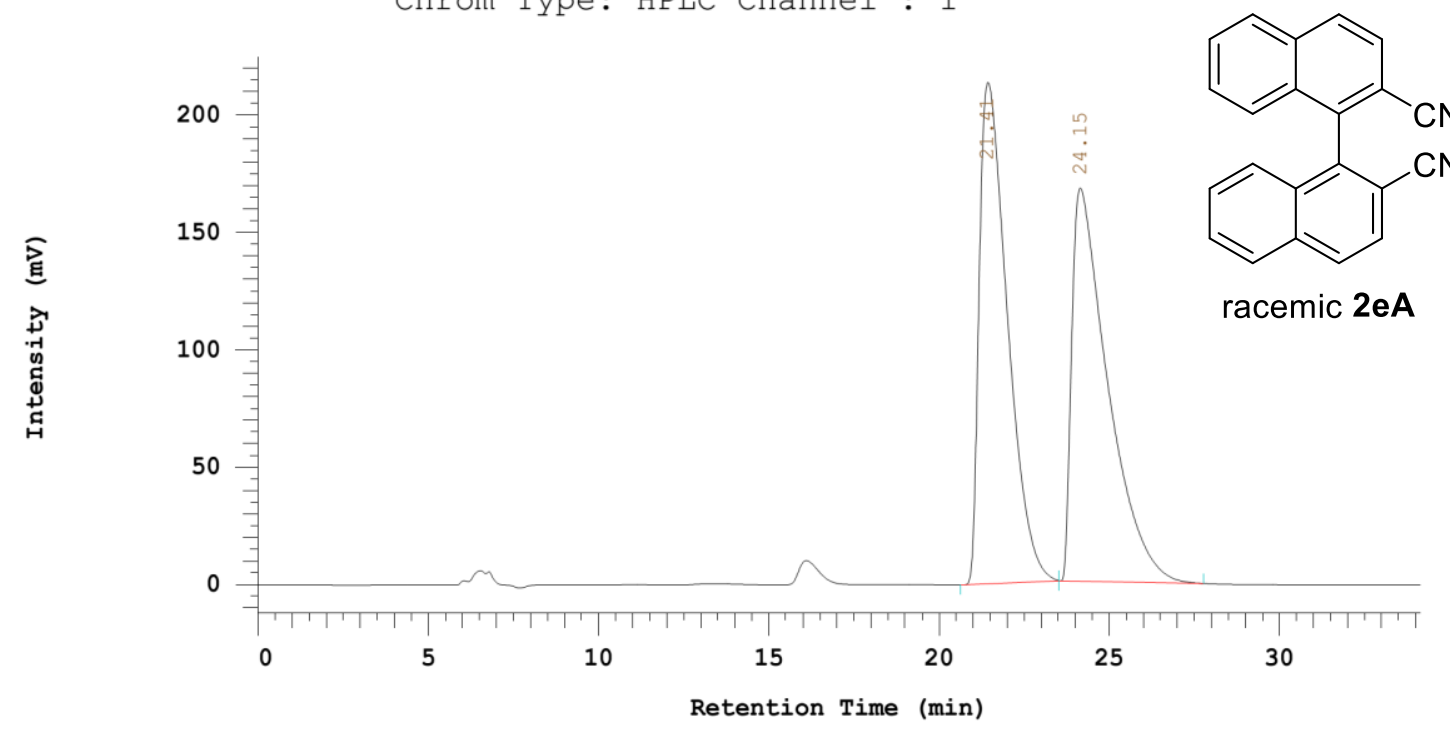

\begin{tabular}{ccccc} 
No. & RT & Area & Conc 1 & BC \\
\hline 1 & 21.41 & 12246123 & 49.868 & MC \\
2 & 24.15 & 12310735 & 50.132 & MC \\
\hline & & 24556858 & 100.000 &
\end{tabular}

Chrom Type: HPLC Channel : 1

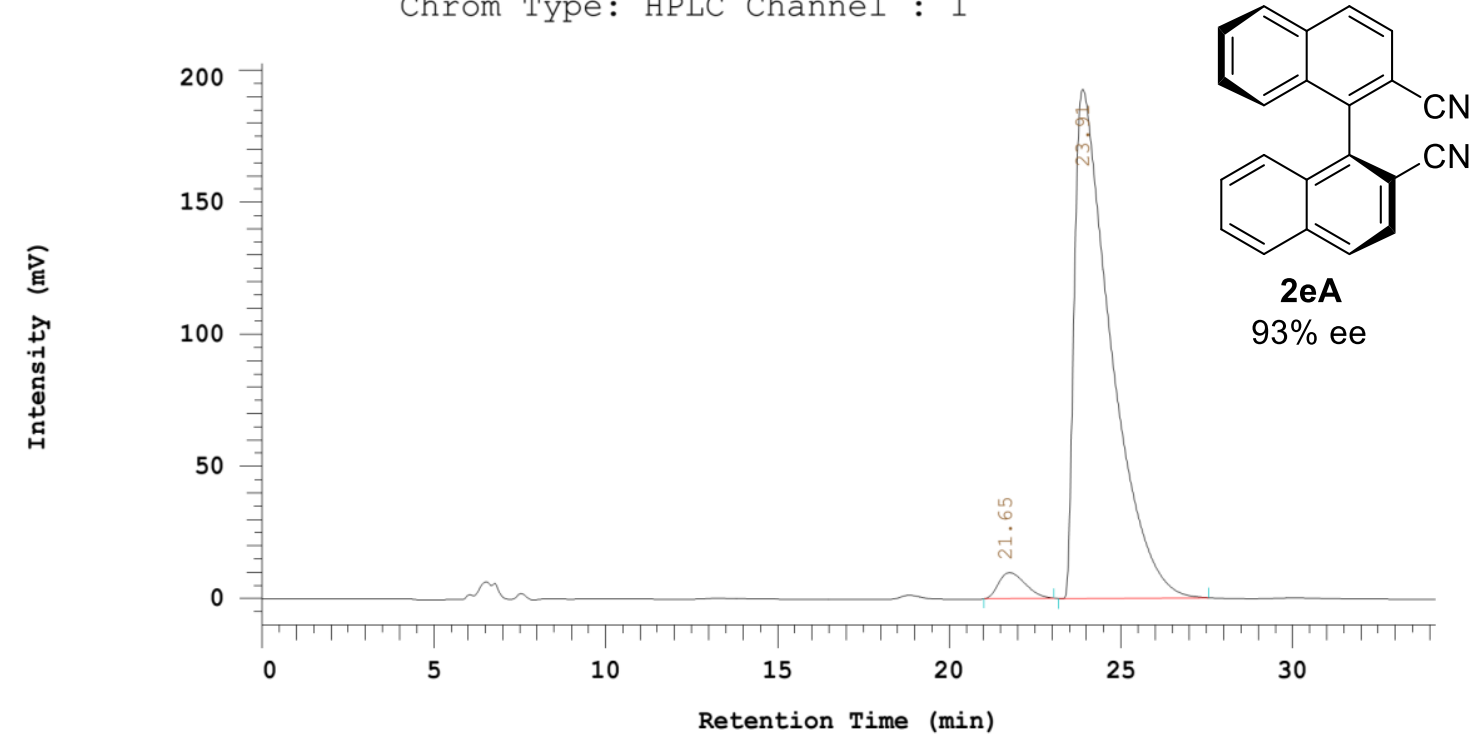

\begin{tabular}{rrrrr} 
No. & RT & \multicolumn{1}{c}{ Area } & Conc 1 & BC \\
\hline 1 & 21.65 & 529033 & 3.598 & MC \\
2 & 23.91 & 14174758 & 96.402 & MC \\
\hline & & 14703791 & 100.000 &
\end{tabular}


Chrom Type: HPLC Channel : 1

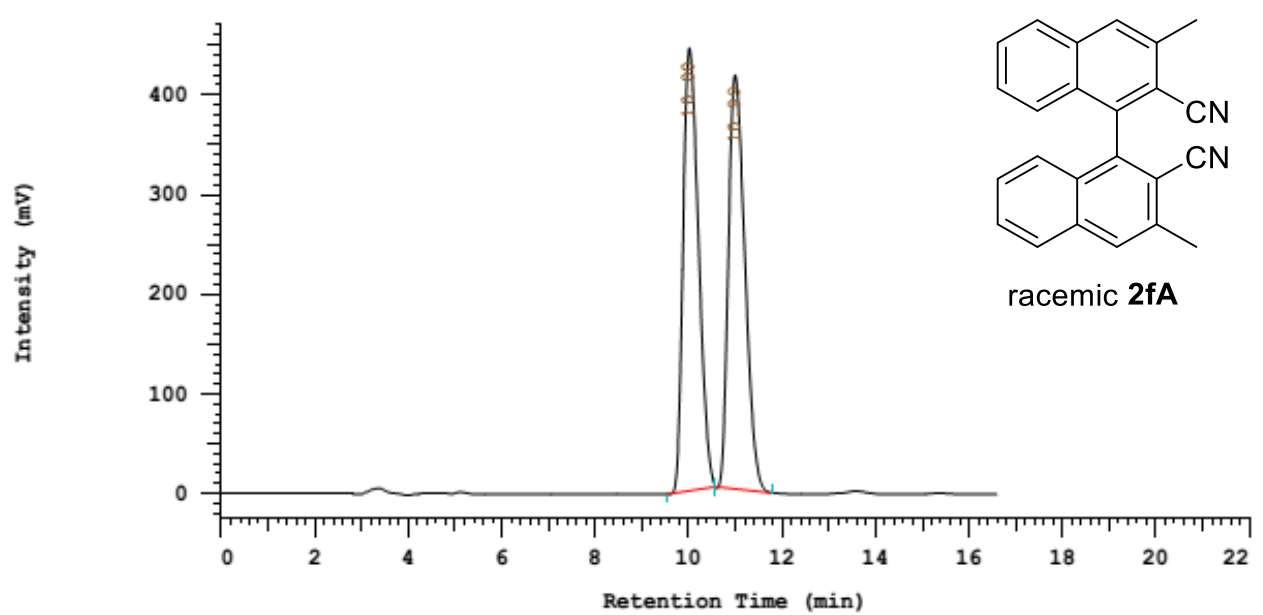

\begin{tabular}{ccccc} 
No. & RT & Area & Conc 1 & BC \\
\hline 1 & 10.00 & 10188931 & 49.996 & MC \\
2 & 10.99 & 10190710 & 50.004 & MC \\
\hline & & 20379641 & 100.000 & \\
\hline
\end{tabular}

Chrom Type: HPLC Channel : 1

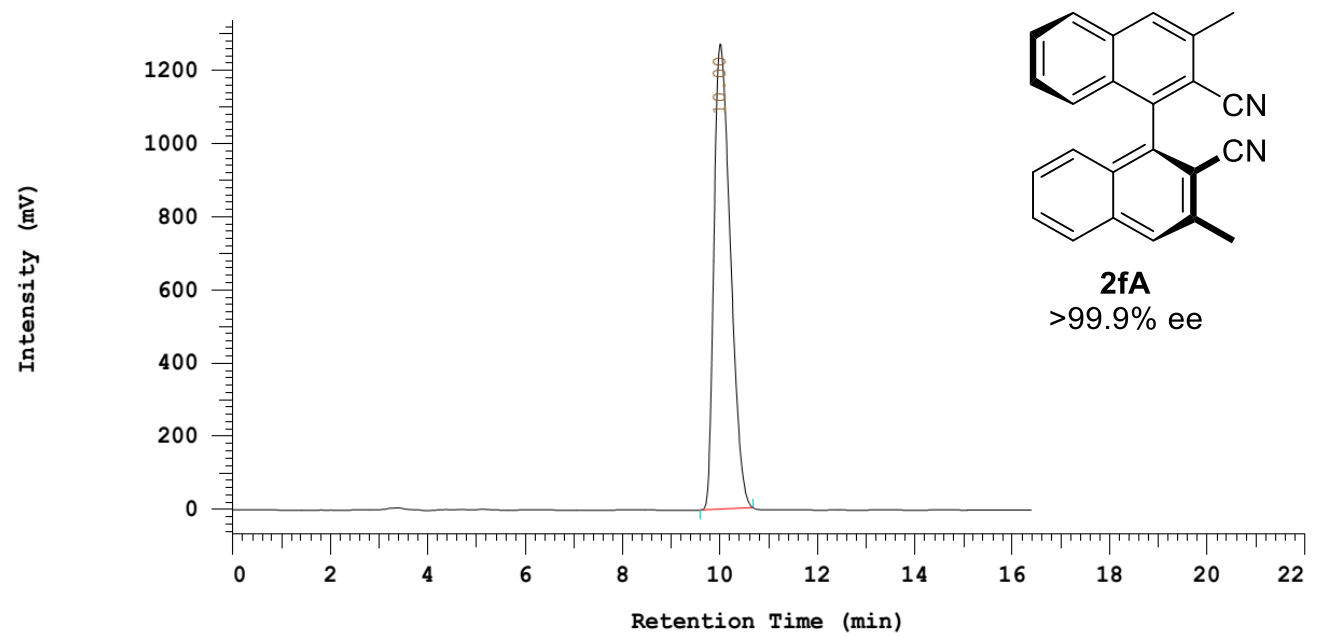

\begin{tabular}{ccccc} 
No. & RT & Area & Conc 1 & BC \\
\hline 1 & 10.00 & 30031849 & 100.000 & MC \\
\hline & 30031849 & 100.000 &
\end{tabular}




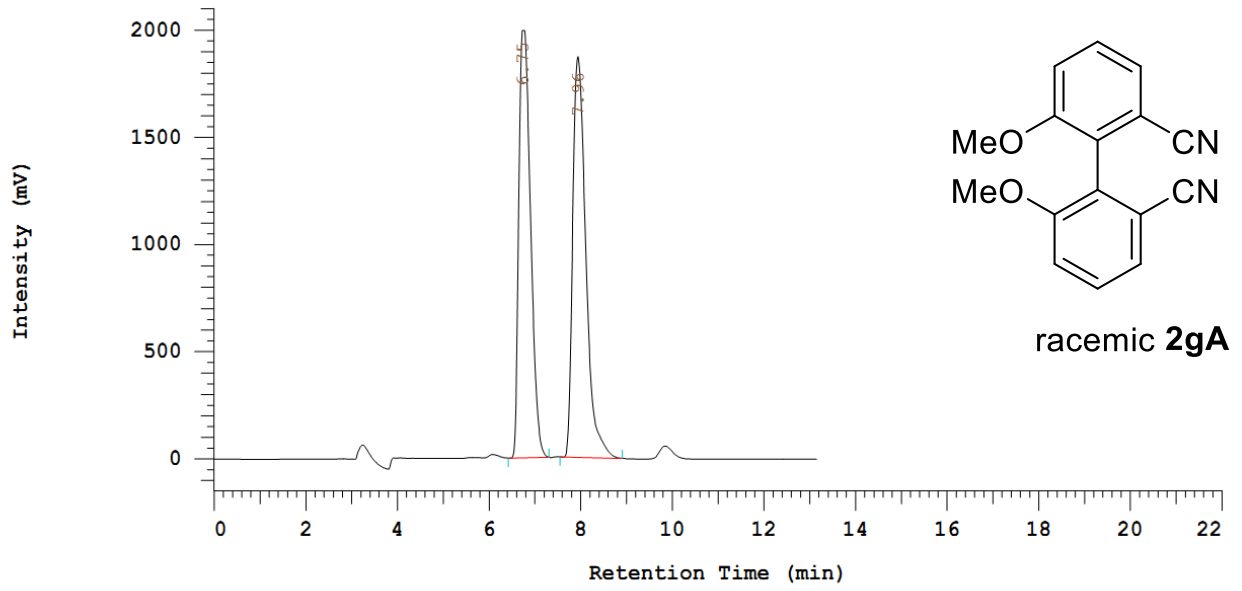

\begin{tabular}{ccccc} 
No. & RT & Area & Conc 1 & BC \\
\hline 1 & 6.75 & 35491507 & 48.847 & MC \\
2 & 7.96 & 37167696 & 51.153 & MC \\
\hline & & 72659203 & 100.000
\end{tabular}

Chrom Type: HPLC Channel : 1

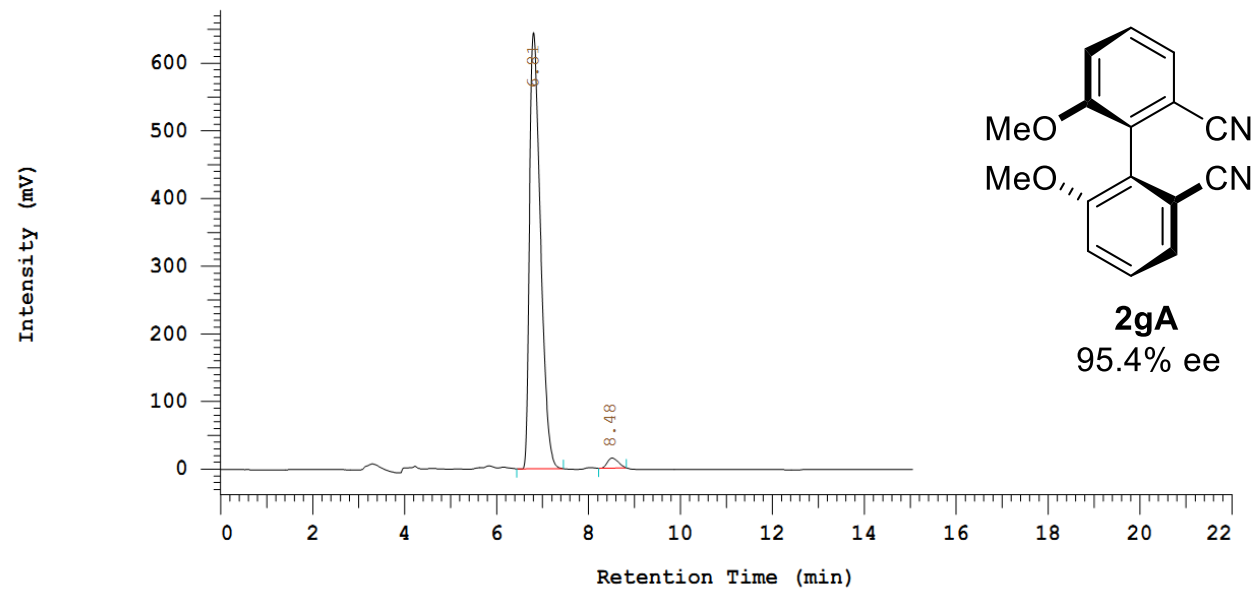

\begin{tabular}{|c|c|c|c|c|}
\hline No. & $\mathrm{RT}$ & Area & Conc 1 & $\mathrm{BC}$ \\
\hline 1 & 6.81 & 10650623 & 97.738 & MC \\
\hline 2 & 8.48 & 246534 & 2.262 & MC \\
\hline & & 10897157 & 100.000 & \\
\hline
\end{tabular}


Chrom Type: HPLC Channel : 1

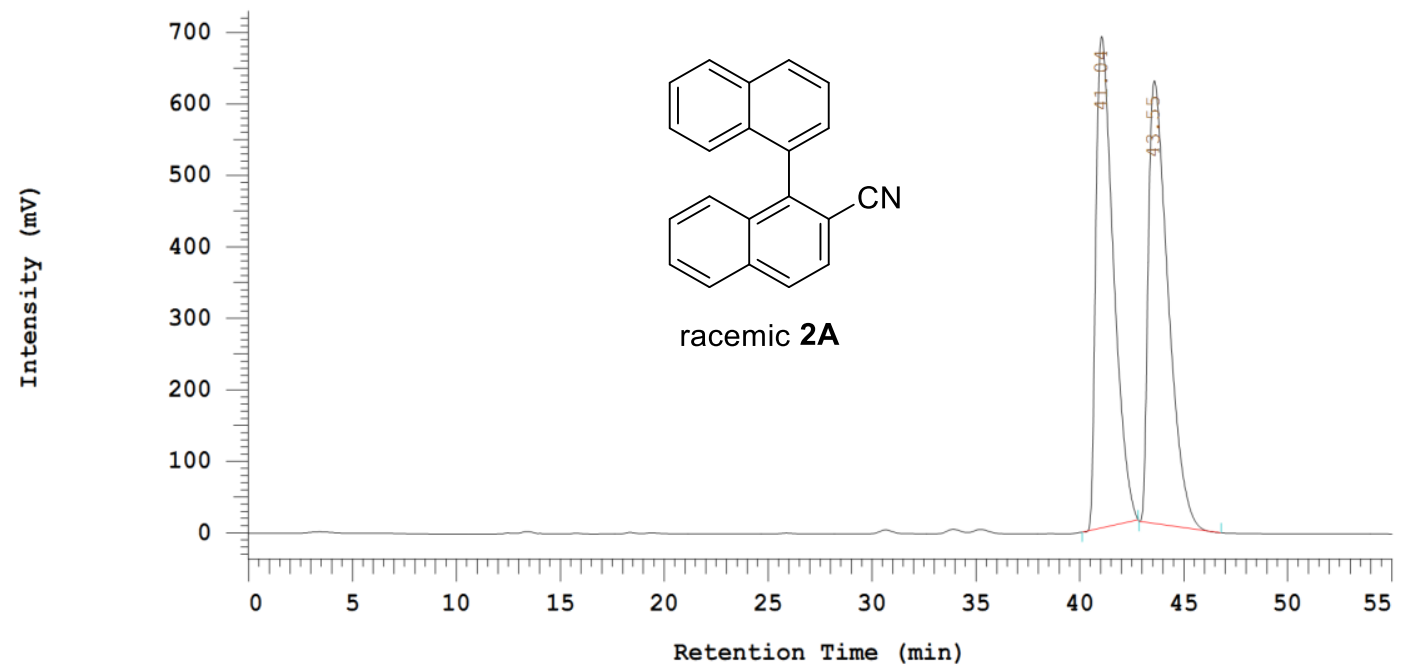

\begin{tabular}{ccccc} 
No. & RT & Area & Conc 1 & BC \\
\hline 1 & 41.04 & 40208044 & 49.604 & MC \\
2 & 43.55 & 40849299 & 50.396 & MC \\
\hline & & 81057343 & 100.000 &
\end{tabular}

Peak rejection level: 0

Chrom Type: HPLC Channel : 1

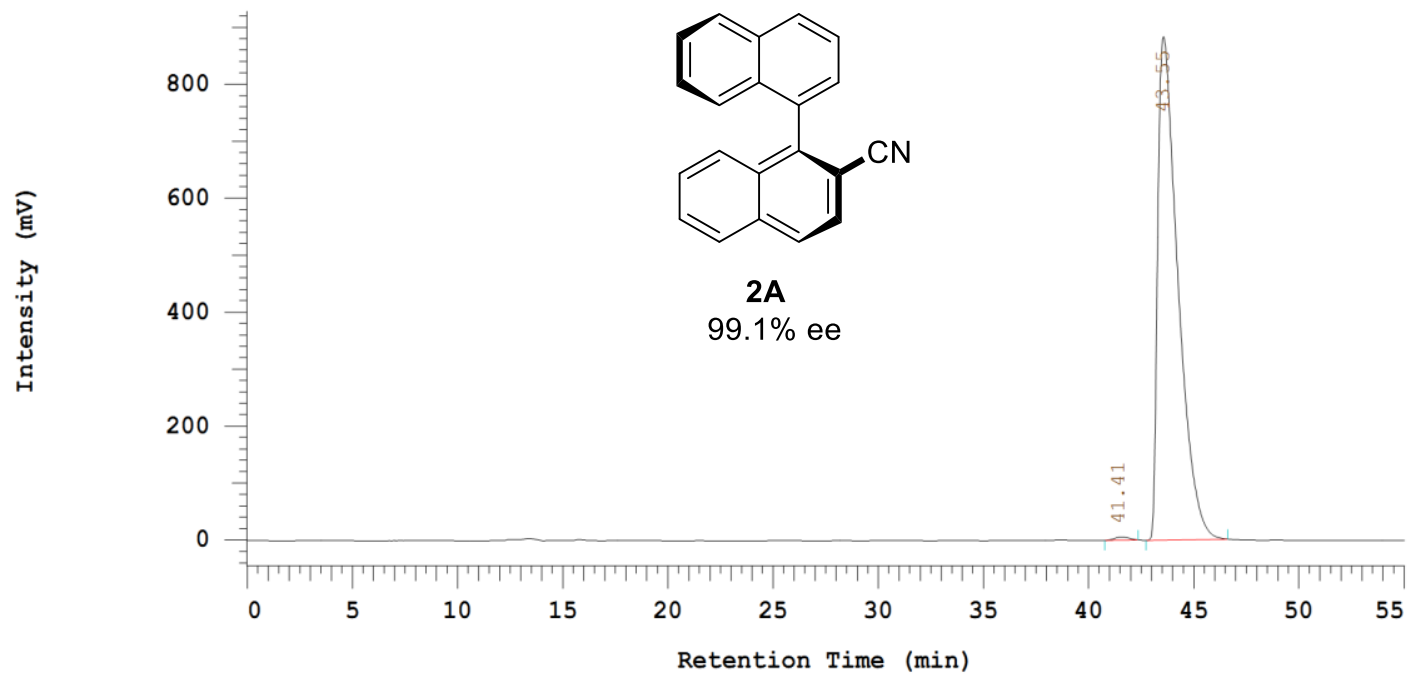

\begin{tabular}{|c|c|c|c|c|}
\hline No. & $\mathrm{RT}$ & Area & Conc 1 & $\mathrm{BC}$ \\
\hline 1 & 41.41 & 268776 & 0.455 & MC \\
\hline 2 & 43.55 & 58824819 & 99.545 & MC \\
\hline & & 59093595 & 100.000 & \\
\hline
\end{tabular}

Peak rejection level: 0 
Chrom Type: HPLC Channel : 1

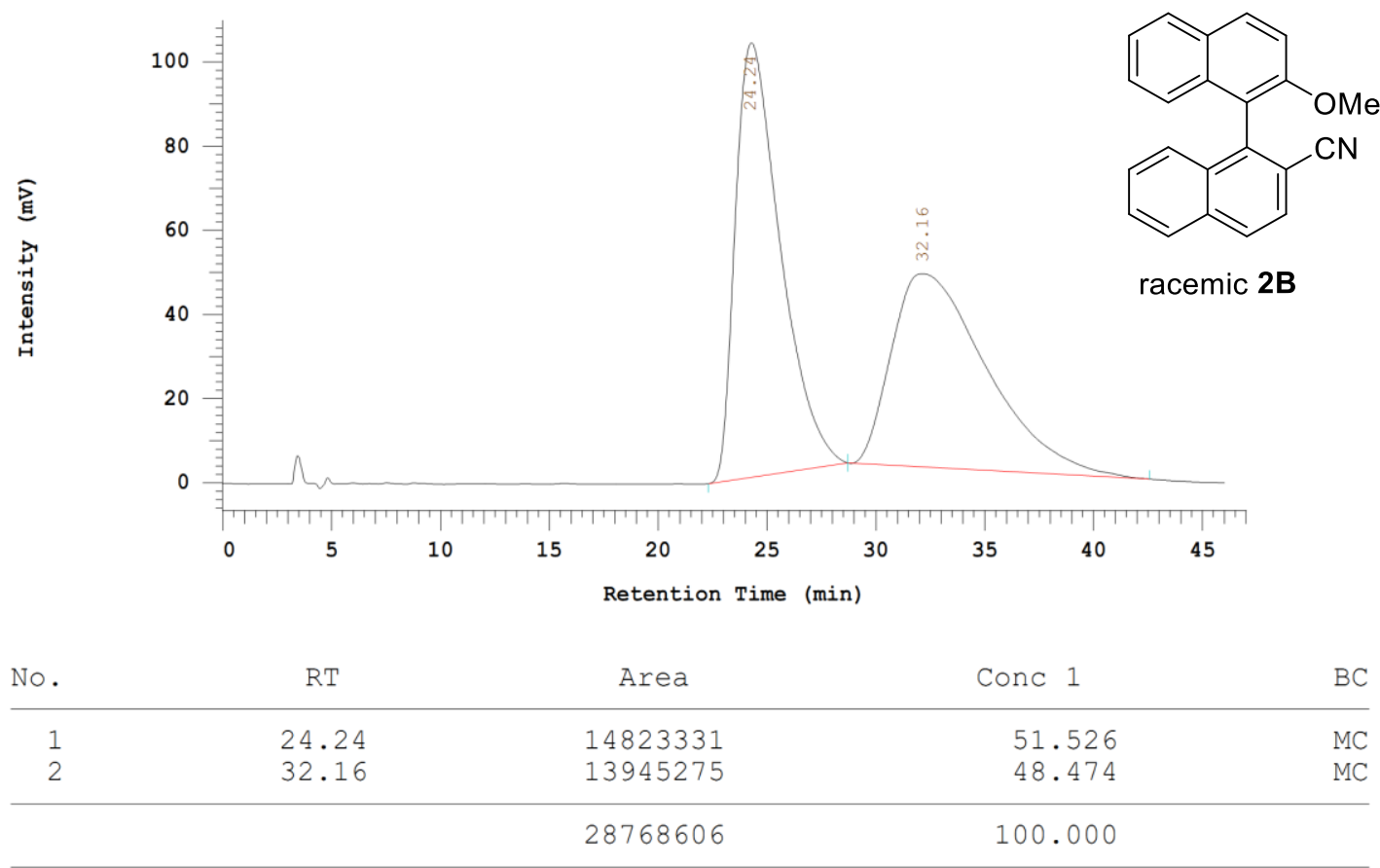

Peak rejection level: 0

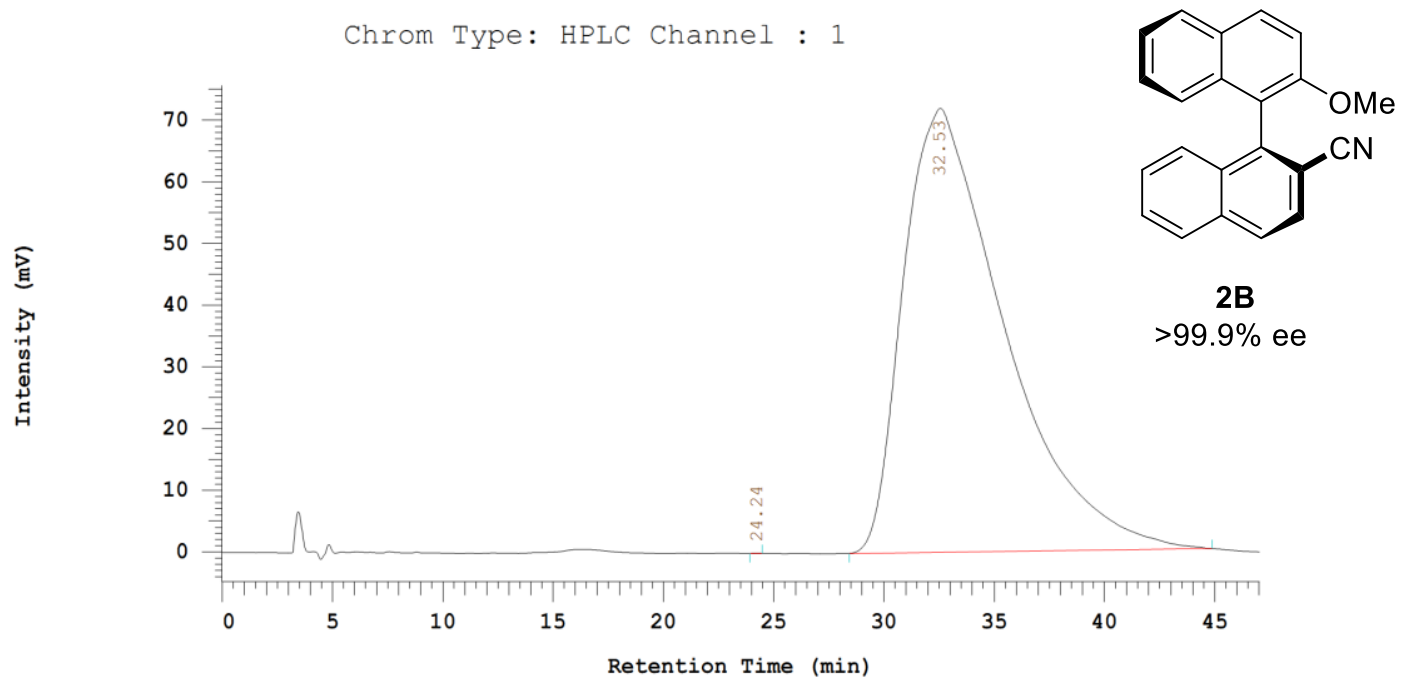

\begin{tabular}{rrrrr} 
No. & RT & Area & Conc 1 & BC \\
\hline 1 & 24.24 & 288 & 0.001 & MC \\
2 & 32.53 & 22986968 & 99.999 & MC \\
\hline & & 22987256 & 100.000 &
\end{tabular}

Peak rejection level: 0 
Chrom Type: HPLC Channel : 1

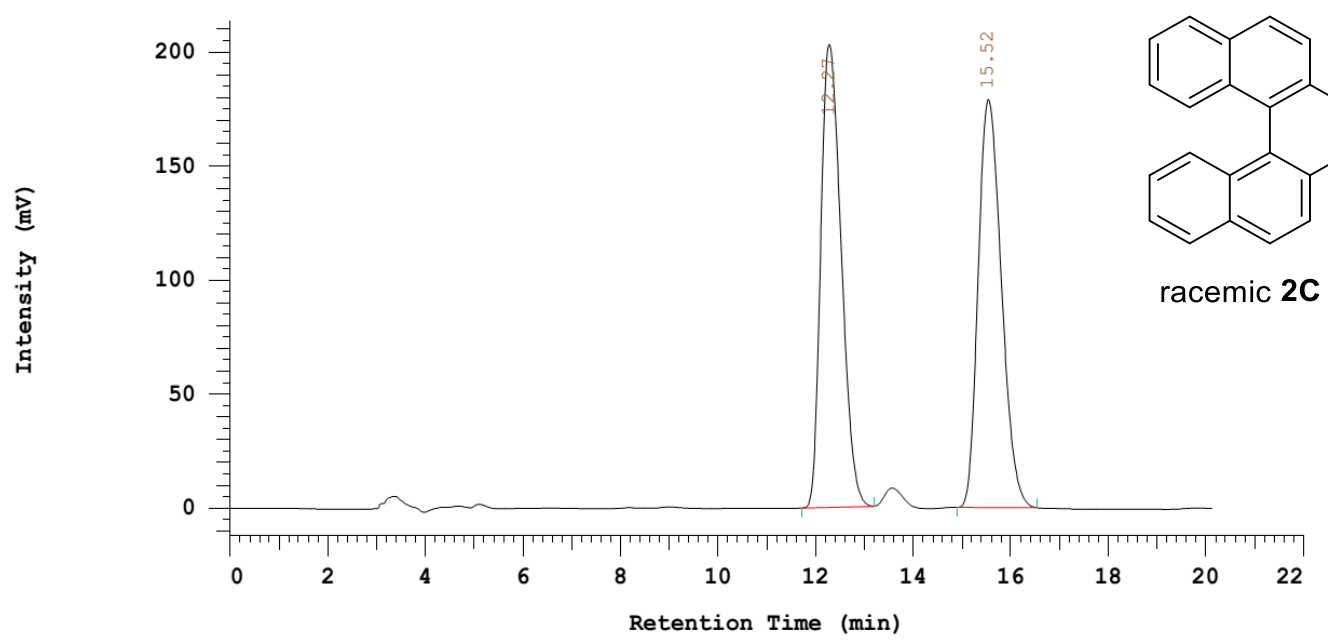

\begin{tabular}{ccccc} 
No. & RT & Area & Conc 1 & BC \\
\hline 1 & 12.27 & 5925166 & 50.065 & MC \\
2 & 15.52 & 5909732 & 49.935 & MC \\
\hline & & 11834898 & 100.000 &
\end{tabular}

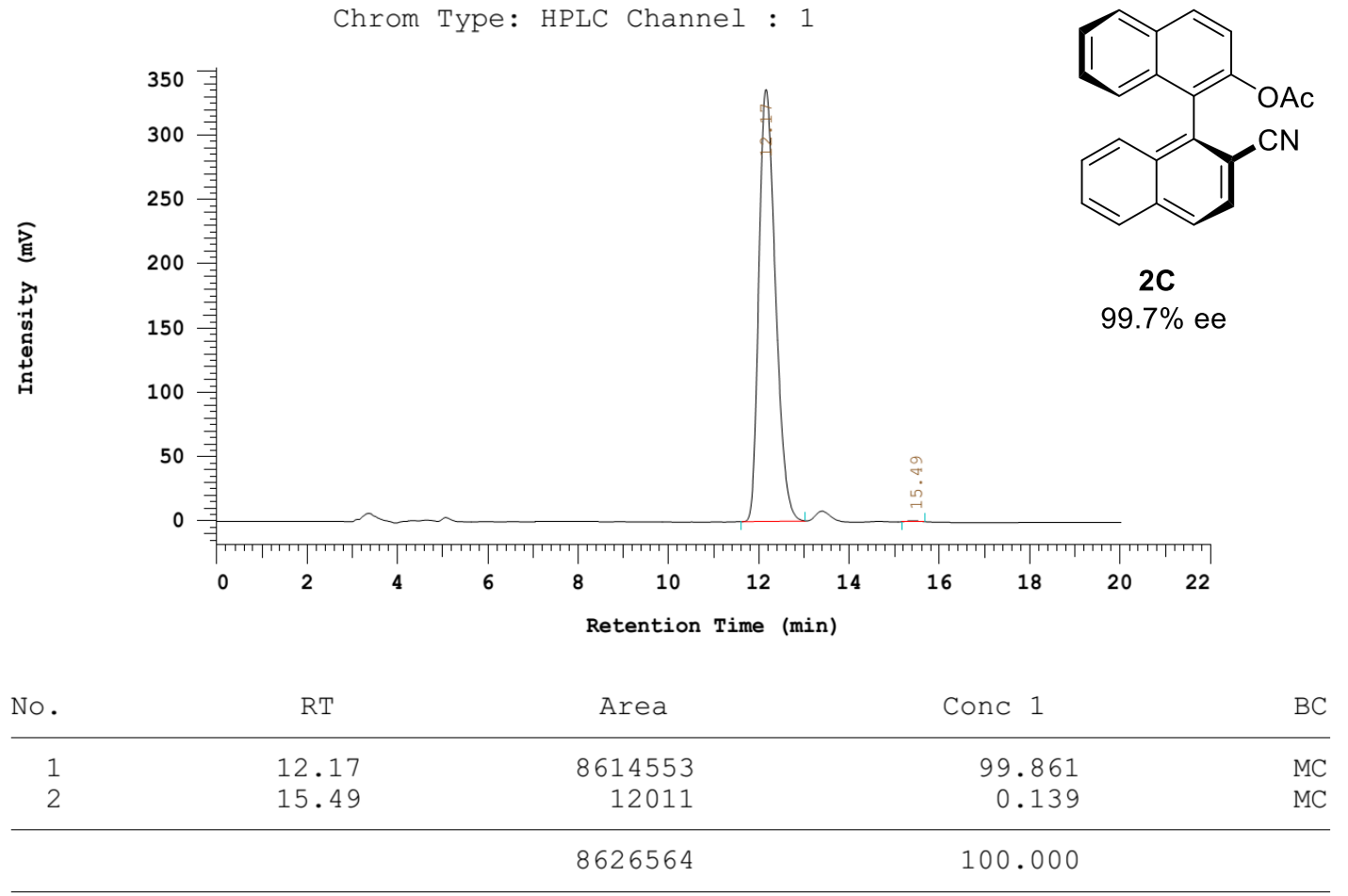

